1991

\title{
Magnetic field strength of toroidal plasma equilibria
}

\author{
David Alan Garren \\ College of William \& Mary - Arts \& Sciences
}

Follow this and additional works at: https://scholarworks.wm.edu/etd

Part of the Nuclear Engineering Commons, and the Plasma and Beam Physics Commons

\section{Recommended Citation}

Garren, David Alan, "Magnetic field strength of toroidal plasma equilibria" (1991). Dissertations, Theses, and Masters Projects. Paper 1539623809.

https://dx.doi.org/doi:10.21220/s2-cjdd-8584

This Dissertation is brought to you for free and open access by the Theses, Dissertations, \& Master Projects at W\&M ScholarWorks. It has been accepted for inclusion in Dissertations, Theses, and Masters Projects by an authorized administrator of W\&M ScholarWorks. For more information, please contact scholarworks@wm.edu. 


\section{INFORMATION TO USERS}

This manuscript has been reproduced from the microfilm master. UMI films the text directly from the original or copy submitted. Thus, some thesis and dissertation copies are in typewriter face, while others may be from any type of computer printer.

The quality of this reproduction is dependent upon the quality of the copy submitted. Broken or indistinct print, colored or poor quality illustrations and photographs, print bleedthrough, substandard margins, and improper alignment can adversely affect reproduction.

In the unlikely event that the author did not send UMI a complete manuscript and there are missing pages, these will be noted. Also, if . unauthorized copyright material had to be removed, a note will indicate the deletion.

Oversize materials (e.g., maps, drawings, charts) are reproduced by sectioning the original, beginning at the upper left-hand corner and continuing from left to right in equal sections with small overlaps. Each original is also photographed in one exposure and is included in reduced form at the back of the book.

Photographs included in the original manuscript have been reproduced xerographically in this copy. Higher quality $6^{\prime \prime} \times 9$ " black and white photographic prints are available for any photographs or illustrations appearing in this copy for an additional charge. Contact UMI directly to order.

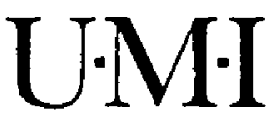

University Microtilms International

A Bell \& Howell Information Company

300 North Zeeb Road, Ann Arbor, MI 48t06-1346 USA

$313 / 761-4700 \quad 800 / 521-0600$ 

- $\quad \ldots$ _

- 
Order Number 9219099

Magnetic field strength of toroidal plasma equilibria

Garren, David Alan, Ph.D.

The College of William and Mary, 1991

Copyright (C1992 by Garren, David Alan. All rights reserved.

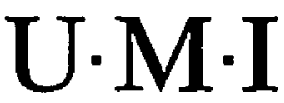

300 N. Zeeb Rd.

Ann Arbor, MI 48106 



\title{
MAGNETIC FIELD STRENGTH OF TOROIDAL PLASMA EQUILIBRIA
}

\author{
A Dissertation \\ Presented to \\ The Faculty of the Department of Physics \\ The College of William and Mary in Virginia \\ In Partial Fulfillment \\ Of the Requirements for the Degree of \\ Doctor of Philosophy
}

by

David Alan Garren

1991 


\section{APPROVAL SHEET}

This dissertation is submitted in partial fulfillment of the requirements for the degree of

Doctor of Philosophy

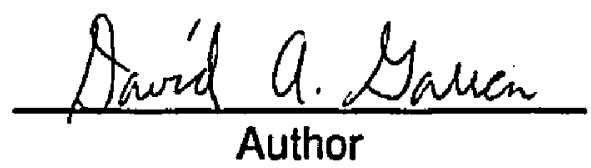

Approved, September 1991

aed $\geqslant 2$
Dr. Allen H. Boozer
- 8va44

Dr. George M. Vahala

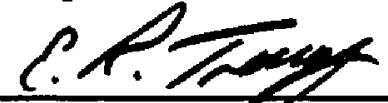

Dr. Eugene R. Ifacy

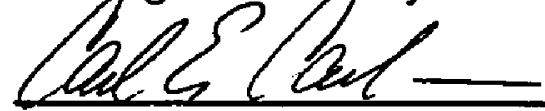

Dr. Carl E. Carlson

Shoerse

Dr. Hugo Woerdeman

Department of Mathematics 


\section{DEDICATED TO MY PARENTS}




\section{TABLE OF CONTENTS}

Page

ACKNOWLEDGEMENTS $\ldots \ldots \ldots \ldots \ldots \ldots \ldots \ldots \ldots \ldots \ldots$ vi

LIST OF FIGURES $\ldots \ldots \ldots \ldots \ldots \ldots \ldots \ldots \ldots \ldots \ldots \ldots \ldots$ vij

ABSTRACT $\ldots \ldots \ldots \ldots \ldots \ldots \ldots \ldots \ldots \ldots \ldots \ldots \ldots$ viii

CHAPTER I. INTRODUCTION . . . . . . . . . . . . . . . . . . 2

CHAPTER II. BOOZER COORDINATES $\ldots \ldots \ldots \ldots \ldots \ldots \ldots \ldots \ldots, 22$

Section II-A. General Curvilinear Coordinates . . . . . . . . . 22

Section II-B. Derivation of Boozer Coordinates . . . . . . . . 26

Section II-C. Particle Drift Trajectories . . . . . . . . . . . 30

Section II-D. Isomorphic Equilibria . . . . . . . . . . . 39

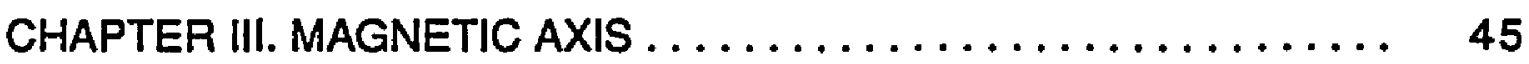

Section III-A. Frenet Unit Vectors . . . . . . . . . . . . . . . 45

Section III-B. Parametric Specification of the Axis . . . . . . 49

Section III-C. Computational Optimization of the Axis . . . . 51

Section III-D. Proof of Two Parameter Optimizations . . . . . . 55

CHAPTER IV. TOROIDAL PLASMA EQUILIBRIA ............. 64

Section IV-A. Basic Equations of Toroidal Plasma Equilibria . 64

Section IV-B. Inverse Method for Defining the Coordinates . . . 70

Section IV-C. Analytic Functions of Boozer Coordinates . . . . . 74

Section IV-D. Expansion about the Magnetic Axis . . . . . . . . 77

Section IV-E. Vacuum Magnetic Fields with Perfect Surfaces . 79

Section IV-F. Force-Free Magnetic Fields . . . . . . . . . . 100

Section IV-G. Scalar-Pressure Plasma Equilibria . . . . . . . . . 108

Section IV-H. Physical Interpretation of Ordering . . . . . . 115

CHAPTER V. RESTRICTIONS ON THE MAGNETIC FIELD STRENGTH . . 122

Section V-A. Non-Vanishing of First Order Curvature Term . . 122

Section V-B. Non-Arbitrariness Through Third Order . . . . . . 123 
Table of contents Continued:

Section V-C. Field Strength Freedom Through Second Order . . 130 Section V-D. Freedom on One Particular Flux Surface . . . . . 132

CHAPTER VI. QUASI-HELICALLY SYMMETRIC EQUILIBIRA . . . . . . 134 Section VI-A. Breaking of Toroidal Isodynamic Equilibria . . . 136 Section VI-B. Breaking of Quasi-Helical Symmetry . . . . . . . 140 Section VI-C. Properties of Approximate Quasi-Heical Symmetry

Section VI-D. Examples of Approximate Quasi-Helical Symmetry 143 $\ldots \ldots \ldots \ldots \ldots \ldots \ldots \ldots \ldots \ldots \ldots \ldots \ldots \ldots$ CHAPTER VII. DISCUSSION $\ldots \ldots \ldots \ldots \ldots \ldots \ldots \ldots \ldots \ldots \ldots 1$

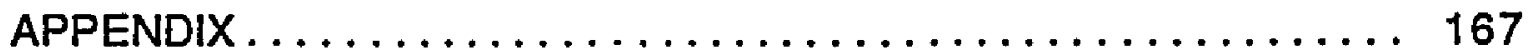
BIBLIOGRAPHY $\ldots \ldots \ldots \ldots \ldots \ldots \ldots \ldots \ldots \ldots \ldots \ldots \ldots \ldots$

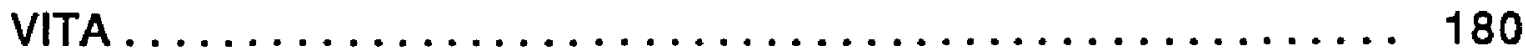




\section{ACKNOWLEDGEMENTS}

The author wishes to express his appreciation to his research advisor, Professor Allen Boozer, for his patient guidance and criticism throughout the author's graduate career. Professor Boozer should also be commended for his tolerance of the author's frequent excursions to Oak Ridge, Atlanta, and Los Angeles. The author would like to thank Professors George Vahala, Gene Tracy, Carl Carlson, and Hugo Woerdeman for their useful comments and for serving on the review committee. The author is also indebted to his fiancée, Penny Shaw, for her useful suggestions in the preparation of this dissertation. 


\section{LIST OF FIGURES}

Figure Page

1. Five Period "Helical-Like" Magnetic Axis . . . . . . . . . 6

2. Boozer Coordinates . . . . . . . . . . . . . . . . . . 9

3. Spatial Position ..................... 10

4. Frenet Unit Vectors $\ldots \ldots \ldots \ldots \ldots \ldots \ldots \ldots \ldots \ldots \ldots \ldots$

5. $X(\psi, \theta, \varphi)$ and $Y(\psi, \theta, \varphi)$ Functions ............... 15

6. Curvature and Torsion of Five Period Helical Axis . . . . . 52

7. Three Period "Knotted" Axis . . . . . . . . . . . . . . . 53

8. Curvature and Torsion of Three Period Knotted Axis .... 54

9. Computationally Selected Axis . . . . . . . . . . . . 56

10. Axis with Optimized Maximum Curvature . . . . . . . 57

11. Constant Curvature Optimized Axis . . . . . . . . . . 58

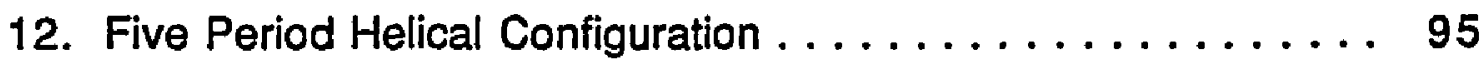

13. Three Period Knotted Configuration . . . . . . . . . 96

14. Ten Period Helical Configuration . . . . . . . . . . . 152

15. Curvature and Torsion of Ten Period Helical Axis . . . . . 153

16. "Tighter" Three Period Knotted Configuration . . . . . . . . . 155

17. Curvature and Torsion of Tighter Knotted Axis . . . . . . . 156

18. Five Period Knotted Configuration. . . . . . . . . . . . 157

19. Curvature and Torsion of Five Period Knotted Axis . . . . . 158 


\section{ABSTRACT}

The goal of nuclear fusion research is to confine a deuteriumtritium plasma at a sufficiently high temperature (15keV) and density $\left(3 \times 10^{20} \mathrm{~m}^{-3}\right)$ for a sufficient length of time $(1 \mathrm{sec})$ to produce net fusion power. One means to attain the required plasma confinement is to embed the plasma within a magnetic field. The global structure of this magnetic field determines the variation of magnetic field strength within the surfaces of constant plasma pressure. This field strength variation in turn determines many of the stability and confinement properties of the plasma.

This dissertation gives the first detailed exposition of the spectrum of possible forms for magnetic field strength corresponding to toroidal plasma equilibria, both within any threedimensional volume and within any two-dimensional surface of constant plasma pressure. Constraints due to the toroidicity of the configuration and the divergence-free property of the magnetic field are found to limit the form of the field strength.

Three-dimensional stellarator equilibria corresponding to a particular form of the magnetic field strength are especially interesting. These "quasi-helically symmetric" equilibria are nonaxisymmetric, toroidal configurations in which the magnetic field strength depends on only one angular coordinate, instead of two, within the constant plasma pressure surfaces. Unlike conventional stellarator equilibria, these quasi-helically symmetric equilibria exhibit the favorable confinement properties of axisymmetric tokamak equilibria. We show that stellarators with exact quasihelical symmetry do not to exist, but that good approximations can be found. 
MAGNETIC FIELD STRENGTH OF TOROIDAL PLASMA EQUILIBRIA 


\section{CHAPTER I}

\section{INTRODUCTION}

The heating of a gas to temperatures over $10^{4}$ Kelvin permits most of the electrons to overcome their atomic binding energies. The global behaviour of such an ionized gas is very different from that of an ordinary gas of particles. We refer to this particular type of gas as a "plasma." The trajectories of the ions and electrons within a plasma are significantly affected by the magnetic and electric fields that they themselves create. Thus, a complete description of the macroscopic properties of a plasma is not conducive to simple analysis.

The global properties of a plasma depend upon many factors, including the temperature and density. The particular class of plasmas that will be examined within this dissertation are relevant to the design of a thermonuclear fusion reactor. In order that fusion reactions occur, the ions of the plasma must have sufficient energy to overcome their repulsive Coulomb potentials. Such a condition is satisfied within a thermal plasma if the temperature of the ions is sufficiently high. Fusion "breakeven" occurs when the energy release from fusion reactions is greater than the rate of energy loss due to bremstrahlung radiation. For deuterium-tritium plasmas, this condition is expressed using Lawson's criterion ${ }^{1}$, which requires 
the plasma number density $n$ and the energy confinement time $\tau_{E}$ to satisfy $n \tau_{E} \geq f(T)$, with $f(T) \approx 3 \times 10^{20} \mathrm{~m}^{-3} \mathrm{~s}$ at a temperature of $T \approx 15 \mathrm{keV}$.

Lawson's criterion is modified for the plasmas of the sun and other stars, since their fusion reactions primarily involve weak nuclear reactions instead of strong nuclear reactions. The particle density and energy confinement time within the hydrogen plasmas of stars must be many orders of magnitude higher than that within the deuterium-tritium plasmas of the laboratory. Stars use their immense gravitational fields to give the required plasma confinement to yield net fusion energy. Obviously, some other means must be applied for laboratory plasmas, such as magnetic confinement.

It is well known that charged particles gyrate about magnetic field lines, obeying the Lorentz force equation, $m\{d v / d t\}=q v \times B$. The radius of gyration, which is called the cyclotron radius, is inversely proportional ${ }^{2}$ to the strength of the magnetic field. Hence, a strong magnetic field can serve to confine the charged particles of a plasma in directions perpendicular to the field lines. Stronger field strength results in better confinement perpendicular to the field lines, since the rate at which the charged particles drift across the field lines is inversely proportional ${ }^{3}$ to the magnetic field strength. However, charged particles with a sufficiently high ${ }^{1}$ Lawson, J. D. (1957). Proc. Phys. Soc. London, Sec. B 70, 6.

${ }^{2}$ Nishkawa, K., and Wakatani, M. (1990). Plasma Physics: Basic Theory wilh Fusion Applications, Springer-Verlag, Berlin, Heidelberg, Germany, p. 15.

3lbid, p. 19. 
velocity parallel to the field lines are not confined in this direction. This particular dilemma can be alleviated by bending the field lines into a toroidal geometry, so that there exist no open ends through which the charged particles can escape.

The direction of gyration of the charged particles about magnetic field lines is always such as to decrease ${ }^{4}$ the strength of the magnetic field $B$. The collective motion of these charged particles yields an electric current $j$ within the plasma. The sum of the Lorentz force, $q \vee \times B$, over all the particles gives a force per unit volume $\mathrm{j} \times \mathrm{B}$. In equilibrium, this force density exactly balances that due to the plasma pressure gradient $\nabla p$. Thus, plasma equilibrium is given by

$$
\mathrm{j} \times \mathrm{B}=\left(1 / \mu_{0}\right)\{\nabla \times B\} \times B=\nabla p,
$$

using Ampere's law $\mathrm{j}=\left(1 / \mu_{0}\right) \nabla \times B$. Eq. (1.1) defines the magnetohydrodynamic 5 (MHD) description of plasma equilibria. Stability requirements usually limit $\beta$, which is the ratio of the plasma pressure $P$ to the magnetic energy density $B^{2} /\left\{2 \mu_{0}\right\}$, to less than ten percent.

A trivial consequence of the plasma equilibrium equation, Eq. (1.1), is

${ }^{4}$ Nishkawa, K., and Wakatani, M. (1990). Plasma Physics: Basic Theory with Fusion Applications, Springer-Verlag, Berlin, Heidelberg, Germany, p. 15.

${ }^{5}$ Friedberg, J. P. (1987) Ideal Magneto-Hydrodynamics, Plenum Press, New York, p. 7. 


$$
B \cdot \nabla p=j \cdot \nabla p=0
$$

Hence, both the magnetic field lines and electric current trajectories lie in surfaces of constant pressure. The "hair" theorem of topology states that the only surface in the three dimensions that can have a finite vector field which is tangent everywhere is a topological torus. Thus, the constant plasma pressure surfaces must be nested toroidal surfaces. The central curve, which generally corrresponds to the maximum plasma pressure, is called the magnetic axis. The shape of this closed, smooth curve determines many of the macroscopic properties of a plasma equilibrium. An example of a five-period, "helical-like" axis is shown in Fig. 1.

A set of magnetic coordinates defined in terms of the pressure surfaces of a plasma equilibrium was developed by Boozer 6 . The derivation and application of these particular coordinates will be presented within Chapter II. The major advantage of implementing Boozer coordinates is that many transport and stability properties of toroidal MHD equilibira can be determined ${ }^{7}$ using only the strength of the magnetic field, $|B(x)|$, expressd in terms of these coordinates. Information regarding the actual direction of the field is of little relevance to plasma stability and confinement. Furthermore, widely differing plasma configurations can have very similar $^{8}$ transport properties, if expressions for their magnetic

\footnotetext{
6Boozer, A. H. (1981). Phys Fluids 24, 1999.

7Boozer, A. H. (1984). Phys. Fluids 27, 2441.

${ }^{8}$ Boozer, A. H. (1983). Phys. Fluids 26, 496.
} 
FIGURE 1

\section{FIVE PERIOD "HELICAL-LIKE" MAGNETIC AXIS}

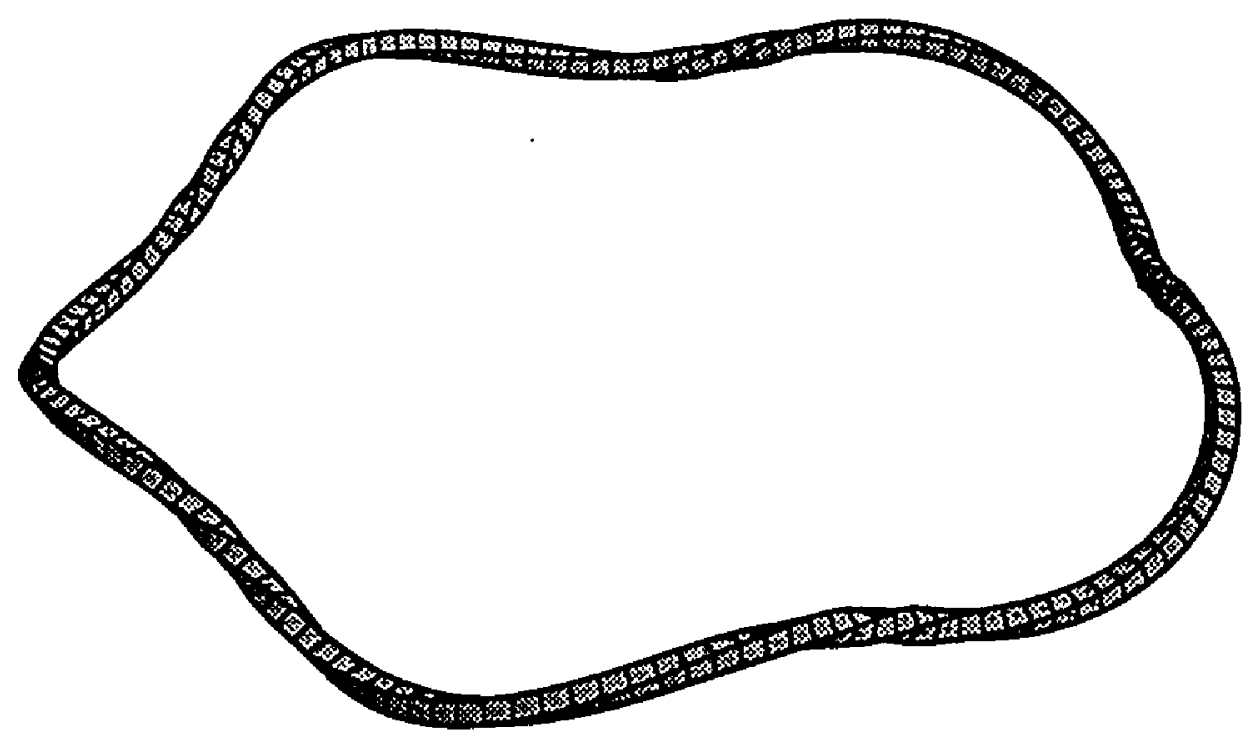

A "helical-like" axis given by Eq. (3.9) with $M=1, N=5$, and $\zeta=1 / 15$. 
field strengths in terms of Boozer coordinates have similar forms.

This dissertation gives the first detailed exposition of the spectrum of possible forms for the magnetic field strength corresponding to toroidal plasma equilibria, both within any threedimensional volume and within any two-dimensional pressure surface. The entire analysis is performed in terms of Boozer coordinates, due to their facility in determining transport and stability properties of plasma equilibria. The particular expansion method that is applied is particularly conducive for such an analysis of the field strength in terms of Boozer coordinates.

Configuration properties other than the magnetic field strength, such as the local and global shear of the magnetic field lines, also affect the stability and confinement properties of general toroidal plasma equilibria. There exist suitable approximations to these parameters for any toroidal plasma equilibrium. The methods developed in this disseratation allow one to investigate the parameter space of optimal toroidal plasma equilibria, using appropriate approximations for the plasma parameters. Thus, we can clarify the variety of three-dimensional toroidal plasma equilibria that are available to the fusion program.

The actual method used to investigate toroidal plasma equilibria involves a Taylor expansion in a radial coordinate and Fourier expansions in two angular coordinates. We implement a Taylor series expansion because plasma stability and transport properties depend primarily on the low order terms of the Taylor 
series. In addition, scalar-pressure MHD equilibria must satisfy the force balance equation, Eq. (1.1), which is non-linear in the magnetic field, making tractable analysis extremely difficult.

The expansion about the axis is actually performed using Boozer coordinates ${ }^{9},\{\psi, \theta, \varphi\}$ (See Fig. 2), due to their facility in determining confinement properties. The toroidal magnetic flux surfaces $\psi$ define the radial coordinate from the magnetic axis to a particular toroidal surface. The plasma pressure, $p$, is a function of $\psi$ alone, so that Eq. (1.2) implies $B \cdot \nabla \psi$, giving field line trajectories within surfaces of constant $\psi$. The poloidal angle $\theta$ defines the "short way" around the toroidal $\psi$-surfaces, and the toroidal angle $\varphi$ defines the "long way" around the toroidal $\psi$-surfaces.

The direct method for determining coordinate surfaces involves specifing the coordinates as a function of position, i.e., $\{\psi(x), \theta(x), \varphi(x)\}$. In this dissertation, we apply the inverse method 10 in which the spatial position is defined with respect ot the coordinates, i.e., $x(\psi, \theta, \varphi)$ (see Fig. 3). Our representation of $x(\psi, \theta, \varphi)$ utilizes the orthonormal Frenet vectors ${ }^{11}$ for space curves, $\left\{\hat{\kappa}_{0}(l), \hat{\tau}_{0}(l), \hat{b}_{0}(l)\right\}$ (see Fig. 4 ) to define the spatial position in the vicinity of the magnetic axis. The length along the axis, $\ell$, of the Frenet vectors is a function of only the toroidal angle, $\varphi$.

The spatial position, $x(\psi, \theta, \varphi)$, is Taylor expanded in terms of ${ }^{9}$ Boozer, A. H. (1981). Phys Fluids 24, 1999.

${ }^{10}$ Garren, D. A., and Boozer, A. H. (1991). Phys. Fivids B: Plasma Physics 3, 2805. 11 Mathews, J., and Walker, R. L., (1970). Mathematical Methods of Physics, W. A. Benjamin, Inc., New York, 2nd ed., p. 408. 
FIGURE 2

BOOZER COORDINATES

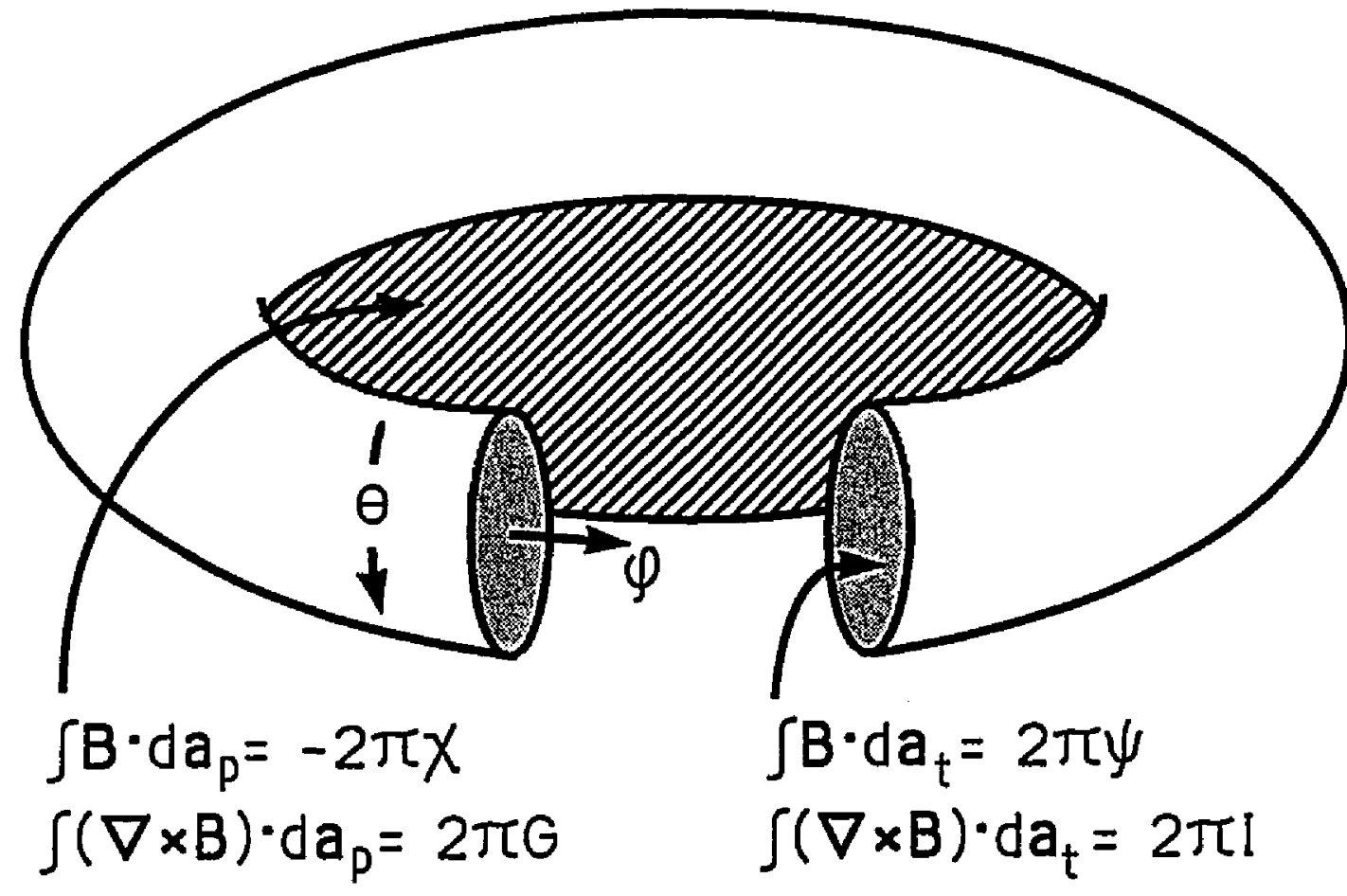

The toroidal flux within a magnetic surface is $2 \pi \psi=\int \mathrm{B} \cdot \mathrm{da} \mathrm{t}_{t}$; the poloidal flux outside of a magnetic surface, i.e., through the hole in the torus, is $2 \pi X(\psi)=-\int B \cdot d a_{p}$; the rotational transform is $l(\psi) \equiv d X / d \psi$, i.e., the poloidal advance per toroidal transit of a magnetic field line is $2 \pi l(\psi)$; the net poloidal "coil" current outside of a constant $\psi$ surface, which gives the toroidal magnetic field, is $2 \pi G(\psi) / \mu_{0}=\left(1 / \mu_{0}\right) \int(\nabla \times B) \cdot d a_{p}$; the net toroidal "plasma" current enclosed by a constant $\psi$ surface, which gives the poloidal magnetic field, is $2 \pi\left[(\psi) / \mu_{0}=\left(1 / \mu_{0}\right) \int(\nabla \times B) \cdot d a_{t}\right.$; the poloidal and toroidal angles are given by $\theta$ and $\varphi$, respectively. 
FIGURE 3

SPATIAL POSITION

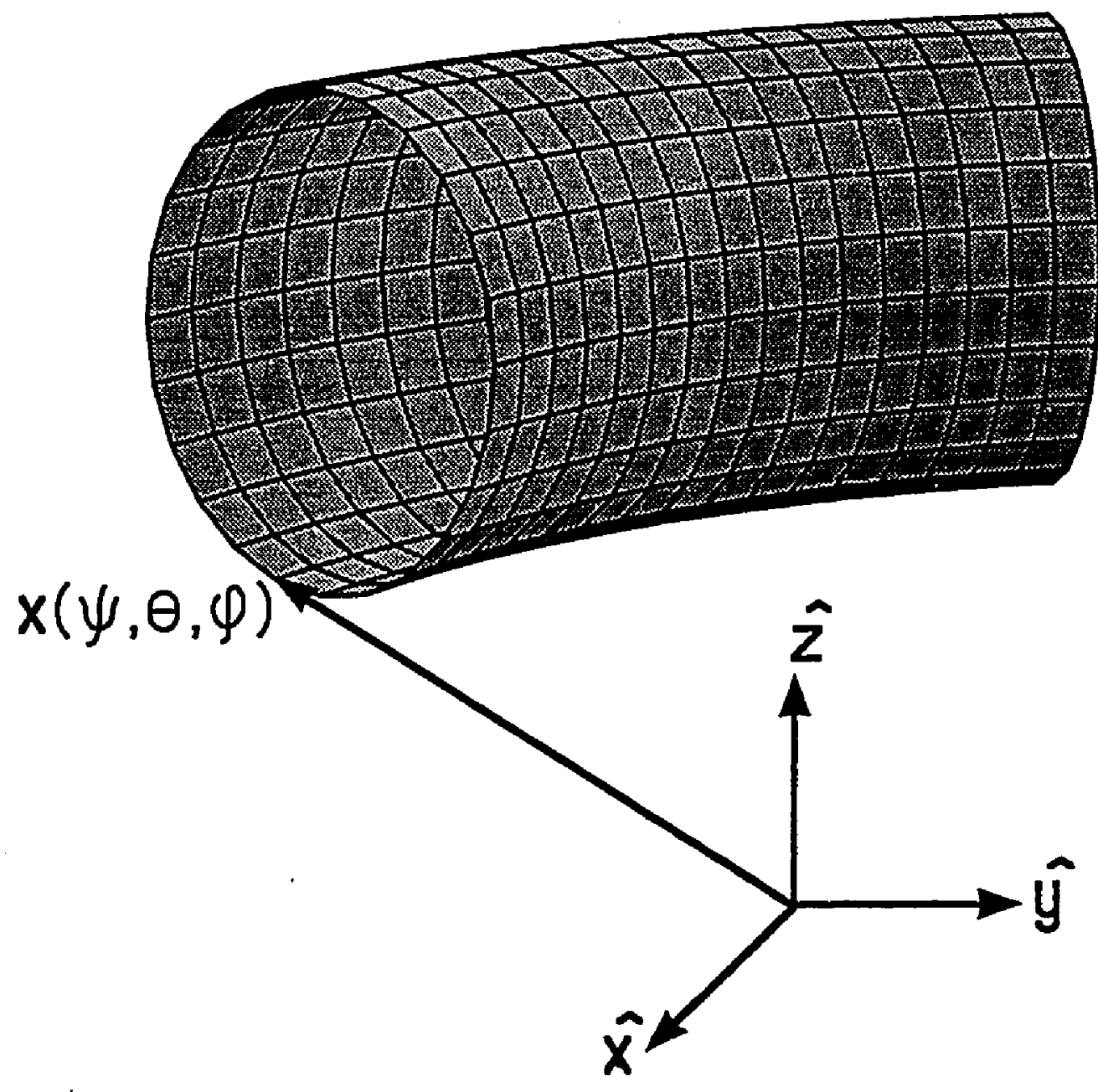

A unique value for the spatial position, $x(\psi, \theta, \varphi)$, with respect to some coordinate system is obtained by specifying the Boozer coordinates $\{\psi, \theta, \varphi\}$. Coordinate surfaces are found by holding the coordinate in question fixed, and varying the other two. 
FIGURE 4

\section{FRENET UNIT VECTORS}

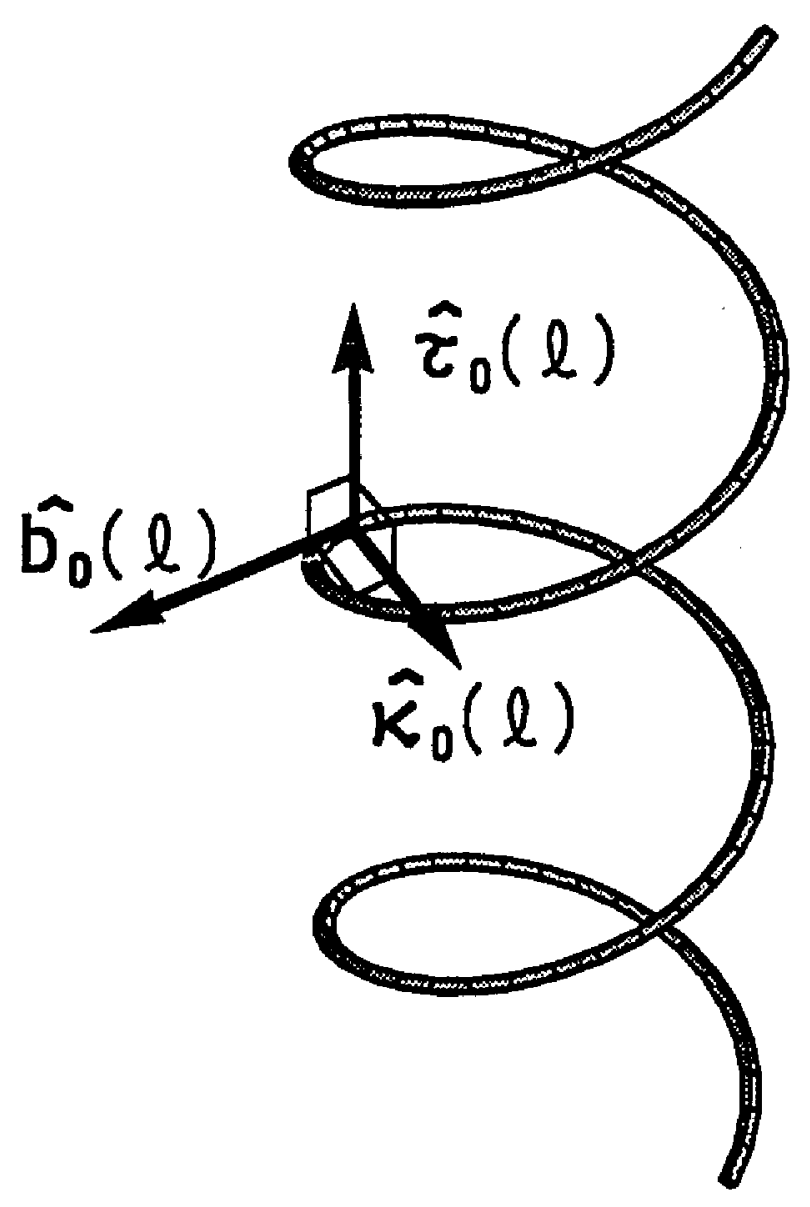

The orthonormal set of Frenet unit vectors $\left\{\hat{k}_{0}(l), \hat{z}_{0}(l), \hat{b}_{0}(l)\right\}$ form a local Cartesian-like coordinate system about a curve; $\hat{b}_{0}(l)$ is the local tangent to the curve; $\hat{k}_{0}(l)$ is the local normal to the curve, i.e., the unit vector lying in the direction of the local curvature; and $\hat{\tau}_{0}(l)$ is the local binormal to the curve that is mutually perpendicular to the other two. 
the square root of the toroidal flux, $\psi^{1 / 2}$, and Fourier expanded in terms of the poloidal angle, $\theta$, and the toroidal angle, $\varphi$. The torodial flux, $\psi$, is approximately $\psi \approx B_{0} \pi r^{2}$, with $r$ the average distance from the magnetic axis to the outermost pressure surface. Hence, the Taylor expansion in the square root of the toroidal flux, $\psi^{1 / 2}$, is equivalent to an expansion in the plasma minor radius, $r$. In normalized units, this procedure is simply a power series expansion in terms of a generalized inverse aspect ratio $\varepsilon \approx r / R_{C}$, with $R_{c}$ the scale length of the magnetic field. The value of $R_{c}$ is chosen to correspond to the minimum of the local radius of curvature of the magnetic axis, so that the expansion parameter, $\varepsilon$, is maximized. This maximization of $\varepsilon$ minimizes the magnitude of the expansion coefficients, thus insuring validity of the expansion throughout the toroidal region.

The direct method for expanding about a magnetic axis was developed by Mercier ${ }^{12}$, Lortz and Nürhenburg ${ }^{13,14,15}$, and Solov'ev and Shafranov ${ }^{16}$, using the toroidal flux $\psi(r, \theta, \ell)$, Taylor expanded in the plasma radius $r$, and Fourier expanded in a poloidal angle $\theta$ and the axis length $\ell$. Lortz and Nürhenburg also used the inverse method to Taylor expand the spatial position $x(\psi, \theta, \varphi)$ in terms of the square root of the toroidal flux, $\psi^{1 / 2}$, and Fourier

12 Mercier, C. (1964). Nucl. Fusion 4, 213.

${ }^{13}$ Lortz, D., and Nührenberg, J. (1976). Z. Naturforsch. 31a, 1277.

${ }^{14}$ Lortz, D., and Nührenberg, J. (1977). Nucl. Fusion 17, 125.

${ }^{15}$ Lortz, D., and Nohrenberg, J. (1979). Z. Naturforsch. 34a, 167.

${ }^{16}$ Solov'ev, L. S., and Shafranov, V. D. (1970). Reviews of Plasma Physics $5,1$. 
expand in terms of a poloidal angle $\theta$ and a toroidal angle $\varphi$, in order to evaluate the Mercier stability criterion. However, their coordinates did not correspond to the Boozer coordinates used within this analysis, so that the stability and transport of their equilibria are not as easily analyzed.

The most valuable product of this dissertation is not the particular expansion method used to examine toroidal plasma equilibria. It is the generic restrictions on the form of the magnetic field strength corresponding to toroidal plasma equilibria. These limitations in the freedom of the field strength confine the spectrum of possible plasma equilibria that are availble to the fusion program.

The expansion of the magnetic field strength corresponding to generic toroidal plasma equilibria is found to have the form ${ }^{17}$

$$
B(\psi, \theta, \varphi)=B_{0}(\varphi)\left\{1+k(\varphi) \times(\psi, \theta, \varphi)+\psi F(\theta, \varphi)+\psi^{3 / 2} C(\theta, \varphi)+\cdots\right\}
$$

This expression is a Taylor series in the square root of the toroidal flux, $\psi^{1 / 2}$, and a Fourier series in the poloidal and toroidal angles, $\theta$ and $\varphi$, respectively. The particular notation in Eq. (1.3) was chosen since the function $F(\theta, \varphi)$ is free and the function $C(\theta, \varphi)$ is constrained, as explained later within this introduction.

The field strength of the magnetic axis axis, $B_{0}(\varphi)$ in Eq. (1.3), is assumed to be non-zero, since the application of Boozer coordinates requires that the magnetic field strength never ${ }^{17}$ Garren, D. A., and Boozer, A. H. (1991). Phys. Fluids B: Plasma Physics 3, 2805. 
vanish 18 within the toroidal region. The function $k(\varphi)$ is the local curvature of the magnetic axis, which is the reciprocal of the magnitude of the local radius of curvature of the magnetic axis. The function $x(\psi, \theta, \varphi)$, which has the form

$$
x(\psi, \theta, \varphi)=\psi^{1 / 2} \rho_{1}(\varphi) \cos \left(\theta-\lambda_{1}(\varphi)\right),
$$

is the lowest order expression for $X(\psi, \theta, \varphi)$ (see Fig. 5), which is the distance from the axis to a constant- $\psi$ surface along the local curvature vector, $\hat{k}_{0}(l)$, of the axis.

The first order variation of the magnetic field strength of Eq. (1.3) is determined entirely by the $k(\varphi) \times(\psi, \theta, \varphi)$ term. This term demonstrates the dominant role of the local curvature of the axis, $K(\varphi)$, in the first order variation of the field strength. The magnetic field strength of Eq. (1.3) shows that a positive (negative) value for $x(\psi, \theta, \varphi)$ in Fig. 5 gives a first order variation in the field strength which is positive (negative). That is, the magnetic field strength is stronger in the direction in which the field lines are curving, and it is weaker in the opposite direction, as one would naively expect. This result is independent of the current and pressure profiles, which give a higher order correction to the magnetic field strength.

The function $\rho_{1}(\varphi)$ of Eq. (1.4), which gives the magnitude of $x(\psi, \theta, \varphi)$, must always be non-zero in order that the lowest order flux surfaces not be completely flattened along the local curvature vector, $\hat{K}_{0}(l)$, which is apparent from Fig. 5 . In the limit in which ${ }^{18}$ Boozer, A. H. (1981). Phys Fluids 24, 1999. 


\section{FIGURE 5}

\section{$X(\psi, \theta, \varphi)$ AND $Y(\psi, \theta, \varphi)$ FUNCTIONS}

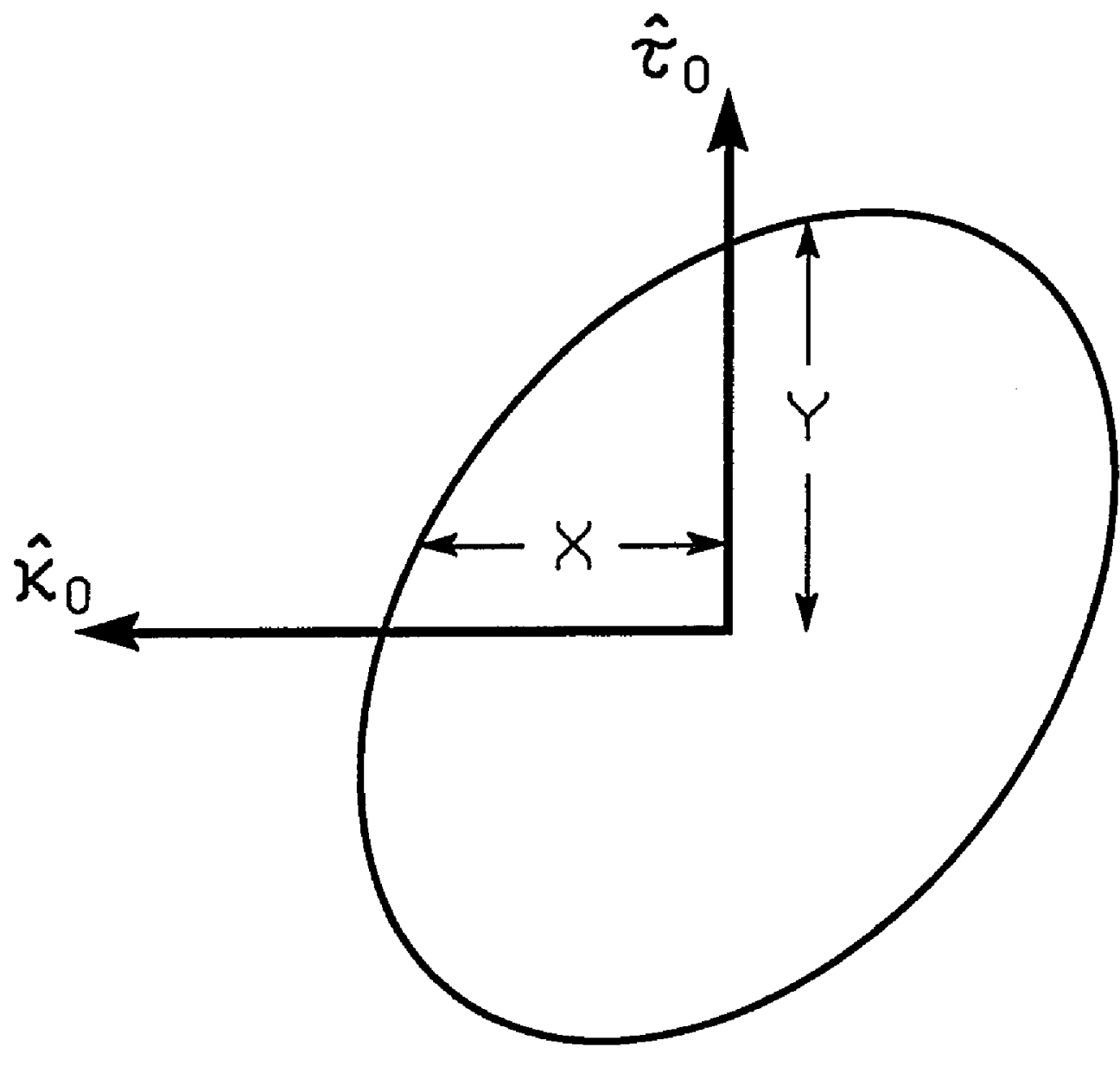

The function $X(\psi, \theta, \varphi)$ is the distance from the magnetic axis along the local curvature vector, $\hat{k}_{0}(\varphi)$, to the magnetic flux surfaces. The function $Y(\psi, \theta, \varphi)$ is the distance from the magnetic axis along the local binormal vector, $\hat{\tau}_{0}(\varphi)$, to the flux surfaces. 
$\rho_{1}(\varphi)$ does vanish, the distance from the axis to the flux surfaces along the local binormal vector, which is given by $Y(\psi, \theta, \varphi)$ in Fig. 5 , diverges, provided that the axis field strength is non-zero. Thus, the magnetic flux surfaces become ribbons that are both infinitely thin and infinitely tall in this singular limit.

The curvature of the axis, $k(\varphi)$, must be non-zero for at least some values of the toroidal angle, $\varphi$, in order to give a toroidal configuration. Together with the necessary non-vanishing of the $\rho_{1}(\varphi)$ function of Eq. (1.4), these restrictions yield one limitation in the freedom of the magnetic field strength. This constraint simply states that the product $K(\varphi) \times(\psi, \theta, \varphi)$ of the first order variation in the field strength of Eq. (1.3) cannot be made to vanish throughout an entire surface of constant $\psi$.

The second order function $F(\theta, \varphi)$ of the magnetic field strength in Eq. (1.3) has the general form

$$
F(\theta, \varphi)=W_{0}(\varphi)+F_{2}(\varphi) \cos \left(2 \theta-\delta_{2}(\varphi)\right)
$$

If the $\varphi$-average of $W_{0}(\varphi)$ of Eq. (1.5) is positive, then the average of the field strength of Eq. (1.3) within a flux surface increases with increasing $\psi$. A plasma configuration having such an average magnetic well ${ }^{19}$ is stable against certain pressure-driven instabilities. In a strict sense, it is the corresponding term within the reciprocal of the square of the field strength, $1 / B^{2}(\psi, \theta, \varphi)$, ${ }^{19}$ Friedberg, J. P. (1987). Ideal Magneto-Hydrodynamics, Plenum Press, Now York, p. 73. 
rather than that within the field strength itself, $B(\psi, \theta, \varphi)$, which is relevant to stability calculations ${ }^{20}$.

Our analysis reveals that the magnetic field strength through second order in $\psi^{1 / 2}$, which is determined by Eqs. (1.3)-(1.5), is nearly arbitrary. Sufficient freedom exists to permit non-trivial, toroidal plasma equilibria corresponding to arbitrary choices of the three second-order, periodic $\varphi$-functions $W_{0}(\varphi), F_{2}(\varphi)$, and $\delta_{2}(\varphi)$ of Eq. (1.5), the two first-order, periodic $\varphi$-functions $\rho_{1}(\varphi)$ and $\lambda_{1}(\varphi)$ of Eq. (1.4), and the one zeroth-order, periodic $\varphi$-function $B_{0}(\varphi)$ of Eq. (1.3). The only proviso is that the product $k(\varphi) \rho_{1}(\varphi)$ of Eqs. (1.3) and (1.4) must be non-zero for at least some values of the toroidal angle, $\varphi$, assuming that the axis field strength, $B_{0}(\varphi)$, does not vanish.

The form of the third-order function $c(\theta, \varphi)$ of the magnetic field strength of Eq. (1.3) is

$$
C(\theta, \varphi)=C_{1}(\varphi) \cos \left(\theta-\gamma_{1}(\varphi)\right)+C_{3}(\varphi) \cos \left(3 \theta-\gamma_{3}(\varphi)\right) .
$$

Our analysis reveals that only one of the two poloidal harmonics of the field strength at third order, i.e., either the $M=1$ or the $M=3$ harmonic of Eq. (1.6), can be chosen freely for arbitrary choices of the field strength through second order. That is, equilibria exist corresponding to arbitrary choices of the zeroth-order function

${ }^{20}$ Nuhrenberg, J., and Zille, R. (1987). Theory of Fuslon Plasmas, Proceedings of the workshop held at Villa Cipressi-Varenna, Italy, Aug. 24-28, Societa ltallana dl Fisica, Bologna, Italy, p. 3. 
$B_{0}(\varphi)$ of Eq. (1.3), the first-order functions $p_{1}(\varphi)$ and $\lambda_{1}(\varphi)$ of Eq. (1.4), the second-order functions $W_{0}(\varphi), F_{2}(\varphi)$, and $\delta_{2}(\varphi)$ of Eq. (1.5), and either the third order $M=1$ poloidal harmonic functions $C_{1}(\varphi)$ and $\gamma_{1}(\varphi)$ or the third order $M=3$ poloidal harmonic functions $c_{3}(\varphi)$ and $\gamma_{3}(\varphi)$ of Eq. (1.6). Again, the only provision is that the product $k(\varphi) \rho_{1}(\varphi)$ of Eqs. (1.3) and (1.4) not vanish for all values of $\varphi$.

In the previous paragraph, we presented the freedom in the magnetic field strength that exists through third order in $\psi^{1 / 2}$. We now give the generic restrictions in the form 21 of the field strength through third and higher orders. The four functions determining the magnetic field strength at third order, i.e., $C_{1}(\varphi)$, $\gamma_{1}(\varphi), C_{3}(\varphi)$, and $\gamma_{3}(\varphi)$ of Eq. (1.6), cannot all be chosen freely, given arbitrary forms of the lower order $\varphi$-functions $B_{0}(\varphi), \rho_{1}(\varphi)$, $\lambda_{1}(\varphi), W_{0}(\varphi), F_{2}(\varphi)$, and $\delta_{2}(\varphi)$ of Eqs. (1.3)-(1.5). This result is a consequence of enforcing equilibrium and the divergence-free property of all magnetic fields. Therefore, even though one may construct forms of the magnetic field strength that give desirable confinement properties, these field strengths may not correspond to actual toroidal plasma equilibria.

We have presented the constraint that the field strength in the three-dimensional volume around the magnetic axis cannot be chosen freely through third order in $\psi^{1 / 2}$. However, the magnetic field strength on one particular plasma pressure surface 22 is nearly arbitrary. This result merely follows from the freedom in the shape 21 Garren, D. A., and Boozer, A. H. (1991). Phys. Fluids B: Plasma Physics 3, 2805. 22 Ibid. 
of the two-dimensional bounding toroidal pressure surface. The only constraint on the field strength within such a flux surface is that a curvature term of the form $k(\varphi) \times(\psi, \theta, \varphi)$, as in Eq. (1.3), must be non-zero for at least some values of $\varphi$. Thus, a particular plasma pressure surface of a toroidal plasma equilibrium can be optimized to have nearly any stability and confinement properties, although those of its interior are more restricted.

We implement these results regarding the freedoms and restrictions of the magnetic field strength to resolve the existence of certain classes of toroidal plasma equilibria. The most desirable class of toroidal plasma equilibria would be those in which the magnetic field strength is constant within the pressure surfaces, i.e., $\mathrm{B}(\psi)$, in terms of Boozer coordinates. The lowest order particle trajectories 23 of such equilibria would always lie within the pressure surfaces. Thus, these toroidal equilibria would have little plasma transport out of the confinement region. Palumbo ${ }^{24}$ was the first to suggest these "isodynamic," or "omnigenous," equilibria. However, Bernardin, Moses, and Tataronis 25 have shown that toroidal, isodynamic equilibria can exist only in limits in which the magnetic field strength of the magnetic axis vanishes or the magnetic flux surfaces become open. Since the symmetry breaking of toroidal isodynamic equilibria occurs at first order in $\psi^{1 / 2}$, rather than some higher order, these equilibria cannot even be well ${ }^{23}$ Palumbo, D. (1968). II Nuovo Cimento X53B, 507.

${ }^{24}$ Ibid.

${ }^{25}$ Bernardin, M. P., Moses, R. W., and Tetaronis, J. A. (1986). Phys. Fluids 29, 2605. 
approximated.

The next most desirable class of toroidal plasma equilibria are those in which the magnetic field strength depends on only one angular coordinate, instead of two, within the pressure surfaces. The magnetic field strength corresponding to such equilibria has the form in Boozer coordinates $B(\psi, \alpha)$, with $\alpha \equiv \theta-N \varphi$ a helical coordinate and $\mathrm{N}$ an integer. This form of the magnetic field strength obviously exists for axisymmetric, i.e., $\varphi$-independent, tokamak equilibria, which give the trivial $N=0$ case. Nonaxisymmetic toroidal equilibria in which the magnetic field strength depends on only one angular coordinate within the flux surfaces were called "quasi-helically symmetric" by Nührenberg and Zille 26 . They computaionally found three-dimensional stellarator equilibria which closely approximate quasi-helical symmetry.

The particle trajectories of quasi-helically symmetric equilibria would be very similar to those of axisymmetric tokamak equilibria27. Thus, these three-dimensional stellarator equilibria would exhibit the desirable confinement properties of axisymmetric tokamak equilibria. Indeed, a stellarator which highly approximates quasi-helical symmetry has few super-banana orbits 28 that give the enhanced transport of traditional stellarators.

The freedom of the magnetic field strength through second order in $\psi^{i / 2}$ permits the existence of quasi-helically symmetric ${ }^{26}$ Nührenberg, J., and Zille, R. (1988). Phys. Lett. A.129, 113.

27 Boozer, A. H. (1983). Phys. Fluids 26, 496.

${ }^{28}$ Beidler, C., et. al. (1990). Fusion Technology: A Journal of the American Nuclear Society 17, 148. 
equilibria through second order ${ }^{29}$. This result can be obtained by setting the functions $B_{0}(\varphi), \rho_{1}(\varphi), W_{0}(\varphi)$, and $F_{2}(\varphi)$ of Eqs. (1.3)(1.5) to constants and requiring the functions $\lambda_{1}(\varphi)$ and $\delta_{2}(\varphi)$ of Eqs. (1.4) and (1.5) to have the form $\lambda_{1}(\varphi)=N \varphi+k_{1}$ and $\delta_{2}(\varphi)=2 N \varphi+k_{2}$, with $k_{1}$ and $k_{2}$ constants. Although there does not exist sufficient freedom to permit quasi-helical symmetry throughout a threedimensional volume through third order in $\psi^{1 / 2}$, the field strength can be made exactly quasi-helical symmetric on one particular pressure surface. The most important flux surface for plasma transport is the surface on which the plasma pressure gradient is a maximum. The enforcement of exact quasi-helical symmetry on such a flux surface causes the symmetry breaking near the axis to scale as $\psi^{1 / 2}$, rather than $\psi^{3 / 2}$.

We can deduce several generic properties 30 of toroidal plasma equilibria which nearly approximate quasi-helical symmetry. First, the curvature of the magnetic axis must never vanish. Second, the toroidal magnetic axis should have a helical-like shape, as opposed to a circular shape. Third, the toroidal variation in the shape of the magnetic flux surfaces should be minimized. Finally, the toroidal plasma configuration should be relatively thin, corresponding to a rather large aspect ratio, $1 / \varepsilon$.

${ }^{29}$ Garren, D. A., and Boozer, A. H. (1991). Phys. Fluids B: Plasma Physics 3, 2822. 30 lbid. 


\section{CHAPTER II}

\section{BOOZER COORDINATES}

In this chapter, the background material relevant to the coordinates developed by Boozer ${ }^{31}$ is presented. These coordinates will be used to investigate, toroidal plasma equilibria throughout this dissertation. In the first section, the theory of general curvilinear coordinates in three dimensions is given. The second section presents a derivation of the particular curvilinear coordinate system developed by Boozer. The third section gives the primary application of Boozer coordinates, which is the dependence of charged particle trajectories ${ }^{32}$ and associated plasma transport on only the strength of the magnetic field expressed in terms of these coordinates. The final section shows the manner in which widely differing plasma configurations can have related ${ }^{33}$ plasma transport properties, if expressions for their magnetic field strengths in terms of Boozer corrdinates have similar forms.

\section{II-A. General Curvilinear Coordinates}

The study of toroidal plasma equilibria suggests the use of a coordinate system in which one of the coordinates corresponds to ${ }^{31}$ Boozer, A. H. (1981). Phys Fluids 24, 1999. 32 Boozer, A. H. (1984). Phys. Fluids 27, 2441. 33 Boozer, A. H. (1983). Phys. Fluids 26, 496. 
the toroidal plasma pressure surfaces. Since a given pressure surface is only constrained to be a topological torus, general curvilinear coordinates must be applied for the fully threedimensional case. For any non-trivial curvilinear coordinate system, there exist two fundamental sets of basis vectors. These two sets are given by the gradients of the coordinates and the cross products of the gradients of the coordinates.

The gradients of the coordinates are related to the partial derivatives of the spatial position via the dual relations of partial differential theory. The dual relations in any $n$-dimensional vector space appear in many differential geometry texts. Only the threedimensional case ${ }^{34}$, which is most relavent to plasma physics, is presented within this dissertation. The dual relations for three curvilinear coordinates $\left\{\xi^{1}, \xi^{2}, \xi^{3}\right\}$ are given by

$$
\begin{gathered}
J \nabla \xi^{2} \times \nabla \xi^{3}=\partial x / \partial \xi^{1}, \\
\left(\partial x / \partial \xi^{2}\right) \times\left(\partial x / \partial \xi^{3}\right)=J \nabla \xi^{1},
\end{gathered}
$$

provided that the spatial Jacobian

$$
J \equiv\left(\partial x / \partial \xi^{1}\right) \cdot\left\{\left(\partial x / \partial \xi^{2}\right) \times\left(\partial x / \partial \xi^{3}\right)\right\}=1 /\left\{\nabla \xi^{1} \cdot\left\{\nabla \xi^{2} \times \nabla \xi^{3}\right\}\right\}
$$

is non-zero and non-infinite within the region of interest. Cyclic permutation of the indices $\{1,2,3\}$ within Eqs. (2.1) and (2.2) also ${ }^{34}$ White, R. B. (1989). Theory of Tokamak Plasmas, North Holland Physics, Amsterdam, p. 6. 
yields valid relations. The dual relations of Eqs. (2.1)-(2.3) will be used to implement the inverse method developed within Chapter IV, which entails expressing the gradients of the coordinates in terms of the partial derivatives of the spatial position with respect to the coordinates.

To simply derive the dual relations of Eqs. (2.1)-(2.3), we begin with the fact that the spatial position $X$ can be specified by three nontrivial coordinates $\left\{\xi^{1}, \xi^{2}, \xi^{3}\right\}$, i.e., $x\left(\xi^{1}, \xi^{2}, \xi^{3}\right)$. If $x$ is written in terms of Cartesian coordinates,

$$
x(x, y, z)=x \hat{x}+y \hat{y}+z \hat{z}
$$

then $\{x, y, z\}$ can be considered functions of $\left\{\xi^{1}, \xi^{2}, \xi^{3}\right\}$, and vice versa. The derivative of one of the coordinates with respect to position is

$$
\partial \xi i(x, y, z) / \partial x \equiv \nabla \xi i(x, y, z)=(\partial \xi i / \partial x) \hat{x}+(\partial \xi i / \partial y) \hat{y}+(\partial \xi i / \partial z) \hat{z}
$$

and the derivative of the position with respect to one of the coordinates is

$$
\partial x\left(\xi^{1}, \xi^{2}, \xi^{3}\right) / \partial \xi j=(\partial x / \partial \xi j) \hat{x}+(\partial y / \partial \xi, j) \hat{y}+(\partial z / \partial \xi j) \hat{z} .
$$

The fact that $\partial \xi^{i} / \partial \xi_{j}$ equals the Kronecker delta, $\delta^{i}{ }_{j}$, and the chain rule imply 


$$
\begin{aligned}
\delta i_{j}=\partial \xi i / \partial \xi j=(\partial \xi i / \partial x)(\partial x / \partial \xi j) & +(\partial \xi i / \partial y)(\partial y / \partial \xi j) \\
& +(\partial \xi i / \partial z)(\partial z / \partial \xi j) .
\end{aligned}
$$

These equations, Eq. (2.7), are called the orthogonality relations and can be written in the condensed form

$$
\delta i_{j}=\nabla \xi_{i} \cdot(\partial x / \partial \xi j) .
$$

The two forms of a three-dimensional vector $B$ in terms of any non-trivial curvilinear coordinate system are the contravariant representation

$$
B_{c o n}=\sum B^{j}\left(\partial x / \partial \xi^{j}\right),
$$

and the covariant representation

$$
B_{\text {cov }}=\sum B_{j} \nabla \xi j .
$$

The orthogonality relations of Eq. (2.8) imply that the coefficients within these two representations are given by

$$
B^{j}=B \cdot \nabla \xi^{j} \text { and } B_{j}=B \cdot(\partial x / \partial \xi j) \text {. }
$$

If the cross product $\nabla \xi^{2} \times \nabla \xi^{3}$ is expanded as a contravariant vector 
and $\left(\partial x / \partial \xi^{2}\right) \times\left(\partial x / \partial \xi^{3}\right)$ as a covariant vector, then evaluation of their components leads to the dual relations of Eqs. (2.1)-(2.3).

\section{II-B. Derivation of Boozer Coordinates}

A set of coordinates that is particularly well suited for transport studies was developed by Boozer ${ }^{35}$. These coordinates permit the magnetic field $B$ consistent with any toroidal plasma equilibirum, Eq. (1.1), to be expressed simultaneously in two simplified forms,

$$
\begin{gathered}
B_{c o n}=\nabla \psi \times \nabla \theta+l(\psi) \nabla \varphi \times \nabla \psi, \\
B_{c o v}=G(\psi) \nabla \varphi+l(\psi) \nabla \theta+B_{*}(\psi, \theta, \varphi) \nabla \psi .
\end{gathered}
$$

These equations for the magnetic field correspond to reduced forms of the contravariant and covariant representations of Eqs. (2.9) and (2.10), respectively. The two simplified forms of the magnetic field of Eqs. (2.12) and (2.13) always exist provided that both the magnetic field strength and the gradient of the plasma pressure never vanish within the toroidal region of interest.

The coordinates used within the two representations of the magnetic field in Eqs. (2.12) and (2.13) are given by $\{\psi, \theta, \varphi\}$ (see Fig. 2). The constant $\psi$ surfaces, which give the toroidal magnetic flux through a cross section of the torus, correspond to the toroidal plasma pressure surfaces. The poloidal angle $\theta$ defines the short way around the torus, and the toroidal angle $\varphi$ defines the long way 35Boozer, A. H. (1981). Phys Fluids 24, 1999. 
around the torus (see Fig. 2).

The contravariant representation of the magnetic field, Eq. (2.12), implies

$$
B \cdot \nabla \psi=0 \text { and } B \cdot \nabla\{\theta-2(\psi) \varphi\}=0 \text {, }
$$

so that magnetic field line trajectories are determined by

$$
\psi=\psi_{0} \text { and } \theta-\imath(\psi) \varphi=\theta_{0}
$$

with $\psi_{0}$ and $\theta_{0}$ constants that determine the particular field line. Hence, the magnetic field lines lie in the toroidal flux, or pressure, surfaces, with the field line twist about the magnetic axis given by the rotational transform, $L(\psi)$.

The covariant form of the magnetic field of Eq. (2.13), satisfies $\{\nabla \times B\} \cdot \nabla \psi=0$, insuring that the current trajectories also lie in the nested flux surfaces. The function $2 \pi G(\psi) / \mu_{0}$ of Eq. (2.13) is the net poloidal "coil" current through the "hole" defined by a toroidal flux surface (see Fig. 2). The function $2 \pi l(\psi) / \mu_{0}$ is the net toroidal "plasma" current through a cross section of a toroidal flux surface. The function $\beta_{*}(\psi, \theta, \varphi)$ is proportional to the plasma pressure gradient, $d p / d \Psi$, as will be explained within Chapter IV.

The derivation ${ }^{36}$ of the two forms of the magnetic field given in Eqs. (2.12) and (2.13) will now be presented. We first assume 36Boozer, A. H. (1981). Phys Fluids 24, 1999. 
that the gradient of the plasma pressure is finite and non-zero everywhere in the toroidal region except on the magnetic axis, which is the degenerate torodial surface. Choose any poloidal $\bar{\theta}$ and toroidal $\varphi$ angles which make the reciprocal of the Jacobian, $\nabla p \cdot\{\nabla \bar{\theta} \times \nabla \varphi\}$, finite and non-zero throughout the toroidal region. Then any three-dimensional vector can be expressed in terms of the general contravariant representation of Eq. (2.9),

$$
B=a(p, \bar{\theta}, \varphi) \nabla p \times \nabla \bar{\theta}+b(p, \bar{\theta}, \varphi) \nabla \varphi \times \nabla p+c(p, \bar{\theta}, \varphi) \nabla \bar{\theta} \times \nabla \varphi
$$

The fact that the magnetic field lines must lie within the pressure surfaces, $B \cdot \nabla p=0$ of Eq. (1.2), shows that the coefficient $c(p, \bar{\theta}, \varphi)=0$. Zero divergence of the magnetic field, $\nabla \cdot B=0$, then implies

$$
(\partial a / \partial \varphi)+(\partial b / \partial \bar{\theta})=0
$$

using the fact that the divergence of crossed gradients is zero. The choice

$$
\begin{array}{r}
a(p, \bar{\theta}, \varphi)=a_{0}(p)\{1+(\partial \omega / \partial \bar{\theta})\}, \\
b(p, \bar{\theta}, \varphi)=b_{0}(p)-a_{0}(p)(\partial \omega / \partial \bar{\theta}),
\end{array}
$$

with $\omega$ a function of all three coordinates, $\{p, \bar{\theta}, \varphi\}$, satisfies Eq. (2.17). Setting $d \psi=a_{0}(p) d p, l(\psi) d \psi=b_{0}(p) d p$, and $\theta=\bar{\theta}+\omega$ then 
permits the general contravariant form of the magnetic field of Eq. (2.16) to be expressed in the reduced form of Eq. (2.12).

In order to derive the other form of the magnetic field, Eq. (2.13), we begin with the general covariant representation of a vector field, Eq. (2.10), or alternatively,

$$
B=\alpha(p, \theta, \varphi) \nabla \varphi+\gamma(p, \theta, \varphi) \nabla \theta+\beta(p, \theta, \varphi) \nabla p
$$

The fact that the electric current trajetories lie in the constant pressure surfaces, $(\nabla \times B) \cdot \nabla p=0$ of Eq. (1.2), implies

$$
(\partial \alpha / \partial \theta)-(\partial \gamma / \partial \varphi)=0
$$

The choice

$$
\begin{aligned}
& \alpha(p, \theta, \varphi)=G(p)+\{G(p)+L(p) I(p)\}(\partial v / \partial \varphi), \\
& \gamma(p, \theta, \varphi)=I(p)+\{G(p)+l(p) I(p)\}(\partial v / \partial \theta),
\end{aligned}
$$

is consistent with Eq. (2.21). We now choose new poloidal and toroidal angles defined by $\theta_{n} \equiv \theta+l(p) v(p, \theta, \varphi)$ and $\varphi_{n} \equiv \varphi+v(p, \theta, \varphi)$, respectively. The transformation from the old angles, $\{\theta, \varphi\}$, to the new angles, $\left\{\theta_{n}, \varphi_{n}\right\}$, permits the general covariant form of the magnetic field in Eq. (2.20) to be expressed in the simplified form of Eq. (2.13). Furthermore, this transformation of the poloidal and toroidal angles does not alter the reduced contravariant form of the magnetic field of Eq. (2.12). Hence, the magnetic field 
corresponding to toroidal plasma equilibria defined in Eq. (1.1) can be simultaneously expressed in the two forms of Eqs. (2.12) and (2.13).

Application of the inner product of the two representations of the magnetic field of Eqs. (2.12) and (2.13) yields ${ }^{37}$

$$
\{\nabla \psi \times \nabla \theta\} \cdot \nabla \varphi=B^{2}(\psi, \theta, \varphi) /\{G(\psi)+L(\psi) I(\psi)\}
$$

The left hand side of Eq. (2.24) is simply the reciprocal of the spatial Jacobian, J, for Boozer coordinates, using Eq. (2.3). This result obviously implies that the spatial Jacobian is inversely proportional to the square of the magnetic field strength,

$$
J(\psi, \theta, \varphi)=\{G(\psi)+2(\psi)](\psi)\} / B^{2}(\psi, \theta, \varphi)
$$

The Jacobian must be non-zero and non-infinite in order to implement the dual relations of Eqs. (2.1) and (2.2), and thus the dual representation of the magnetic field of Eqs. (2.12) and (2.13). Therefore, the magnetic field strength must be non-zero within the toroidal region in order to apply Boozer coordinates.

\section{II-C. Particle Drift Trajectories}

Plasmas of primary interest to fusion research lie in the low collisionality regime. This fact follows since the mean free path of 37 Boozer, A. H. (1981). Phys Fluids 24, 1999. 
the plasma particles is on the order of $10^{4}$ meters, whereas the size of most confinement devices is only a few meters. Hence, the transport of energy and particles within these plasmas is dominated by the trajectories of charged particles within the toroidal magnetic field. For sufficiently strong magnetic field strength, a charged particle gyrates about a magnetic field line in a small orbit compared to the scale of the magnetic field. The motion parallel to the magnetic field lines is relatively unconstrained, so that most of the charged particles circulate within the toroidal magnetic configuration along the field lines thousands of times before being affected by collisions. A small percentage of the particles execute "trapped" orbits which do not transit the torus poloidally.

To a lowest order approximation, the charged particles within a plasma merely follow the magnetic field lines themselves. The center of a particle's circular path in the plane perpendicular to the field lines is called its "gyro-center." To lowest order, a charged particle's gyro-center merely corresponds with the field line about which it is gyrating. To first order in the particle gyro-radius to the scale length of the magnetic field, there exist small drifts of the particles in directions perpendicular to the magnetic field lines. These drifts largely determine the transport of energy and particles out of the confinement region of a low collisionality plasma. The calculation of these particle drifts for a fully three-dimensional configuration is extremely arduous using an arbitrary coordinate system. Additionally, transport across the plasma pressure 
surfaces is dificult to assess using non-flux coordinates.

The implementation of Boozer coordinates ${ }^{38}$ circumvents many of the impediments of calculating plasma transport. The primary application of using these coordinates is that the lowest order expression for the particle drift trajectories ${ }^{39}$ depends on the particular magnetic configuration only through the form of the magnetic field strength expressed in terms of these coordinates. The methods of Hamiltonian mechanics are used in the derivation of the expression for the particle drift trajectories.

Boozer derives the lowest order Hamiltonian governing particle drift trajectories within a toroidal plasma equilibrium,

$$
H\left(\theta, p_{\theta}, \varphi, p_{\varphi}\right)=(1 / 2) m\left\{v_{\|}\right\}^{2}+\mu B+e \Phi,
$$

with $m$ the particle mass, $e$ its charge, $v_{\|}$the particle velocity parallel to the magnetic field, $B(\psi, \theta, \varphi)$ the magnetic field strength, and $\Phi(\psi, \Theta, \varphi)$ the electric potential. The parameter

$$
\mu \approx\left\{m\left\{v_{\perp}\right\}^{2}\right\} /\{2 B\}
$$

of Eq. (2.26) is called the particle's "magnetic moment." This name follows using the value for the magnetic moment, $\mu=I \pi r^{2}$, due to a circular loop of radius $r$ and electric current 1 . The time-averaged electric current created by the gyrating particle is given by ${ }^{38}$ Boozer, A. H. (1981). Phys Fluids 24, 1999. ${ }^{39}$ Boozer, A. H. (1984). Phys. Fluids 27, 2441. 
$I=e \omega /\{2 \pi\}$, with $\omega$ the angular frequency of gyration. Approximating $\omega$ and $r$ by the cycloton frequency $\omega=e B / m$ and the cyclotron radius $r=v_{\perp} / \omega$ for a charged particle within a uniform magnetic field then yields Eq. (2.27).

Kruskal 40 has shown that the magnetic moment is conserved extremely accurately, provided that the particle gyroradius is small compared to the scale of the curvature of the magnetic field lines. This adiabatic invariance of $\mu$ follows since it is merely the "action" $11 \oint p d q$ of Hamiltonian mechanics corresponding to the particle gyration about a field line. Here, the coordinate $q$ gives the angle of rotation about the field line and $P$ is its canonical momentum.

The Boozer coordinate angles $\theta$ and $\varphi$ are the two canonical variables of the drift Hamiltonian of Eq. (2.26). The momenta canonically conjugate to these two angles are

$$
\begin{gathered}
P_{\theta}=\{m I(\psi) / B(\psi, \theta, \varphi)\} v_{\|}+e \psi, \\
P_{\varphi}=\{m G(\psi) / B(\psi, \theta, \varphi)\} v_{\|}-e x(\psi),
\end{gathered}
$$

with $X(\psi)$ the poloidal magnetic flux through the central hole of a constant pressure surface (see Fig. 2). The poloidal flux is related to the rotational transform $l(\psi)$ by the relation $l(\psi)=d X / d \psi$. The guiding-center drift trajectories are obtained by integrating ${ }^{40}$ Kruskal, M. (1957). Rendiconti del Terzo Congresso Internazionale sui Fenomeni D'lonizzazione nei Gas tenuto a Venazia, Societa llaliana di Fisica, Milan, p. 56.

${ }^{41}$ Goldstein, H. (1980). Classical Mechanics, Addison-Wesley Publishing Company, Inc., Philippines, 2nd ed., p. 366. 
Hamilton's equations

$$
\begin{aligned}
\dot{\theta}=\left(\partial H / \partial p_{\theta}\right), & \dot{\varphi}=\left(\partial H / \partial p_{\varphi}\right), \\
\dot{p}_{\theta}=-(\partial H / \partial \theta), & \dot{p}_{\varphi}=-(\partial H / \partial \varphi),
\end{aligned}
$$

with the overdots denoting time derivatives. Differing trajectories within $\left\{\theta, p_{\theta}, \varphi, p_{\varphi}\right\}$ space are obtained by varying the value of the magnetic moment, $\mu$, and the initial values for the canonical coordinates and momenta. The reduction of this Hamiltonian mechanics problem from a system of six variables- $\theta, p_{\theta} \varphi, p_{\varphi}, \mu$, and the gyrophase-to a sytem of four variables follows from the adiabatic invariance of $\mu$ and the corresponding irrelevance of the gyrophase.

A set of $\left\{\theta, P_{\theta}, \varphi_{,} P_{\varphi}\right\}$ at some given time determine the spatial position of a charged particle's guiding center relative to the Boozer coordinates, $\{\psi, \theta, \varphi\}$, of the particular magnetic configuration. The position of a particle relative to the radial coordinate $\psi$ is determined by the values of $\left\{\theta, p_{\theta}, \varphi, p_{\varphi}\right\}$. This result can be obtained by solving for $\psi$ implicitly using Eqs. (2.28) and (2.29). For a vacuum magnetic field, the toroidal electric current, $I(\psi)$, vanishes, so that Eq. (2.28) trivially reduces to $P_{\theta}=e \psi$. Thus, the canonical momentum, $P_{\theta}$, gives the location of the guiding-center relative to the toroidal flux surfaces for vacuum fields.

For most plasmas of interest to fusion research, the electric potential, $\Phi(\psi, \theta, \varphi)$ in Eq. (2.26), is approximately uniform within a 
constant- $\psi$ or pressure surface, thus giving $\phi(\psi)$. This result follows from the high mobility of the electrons in the direction parallel to the magnetic field lines. Hence, the poloidal and toroidal variation of the magnetic field strength expressed in terms of Boozer coordinates largely determines the particle drift trajectories within a given magnetic configuration via Eqs. (2.26) and (2.28)-(2.30).

The form of the magnetic field strength, $B(\psi, \theta, \varphi)$, corresponding to toroidal plasma equilibria cannot be chosen freely, as will be shown in Chapter V. These restrictions in the form of the magnetic field strengh are the primary results of this dissertation. These field strength limitations imply that the drift Hamiltonian of Eq. (2.26) lacks the arbitrary freedom that could be trivially used give desirable drift trajectories. For this reason, the restrictions on the form of the magnetic field strength constrain the freedom of the transport properties corresponding to general toroidal plasma equilibria.

A derivation of the particle drift Hamiltonian of Eq. (2.26) will now be presented. We will assume that the magnetic field is stationary in time and that the plasma pressure function gives perfectly nested toroidal surfaces. This derivation 42 can be generalized to apply for slowly changing magnetic fields with islands structures and stochastic field lines, although these effects are not relevant to the assumptions made throughout this dissertation.

${ }^{42}$ Boozer, A. H. (1984). Phys. Fluids 27, 2441. 
To derive the drift Hamiltonian of Eq. (2.26), we begin with the exact Lagrangian of a particle in a stationary magnetic and electric field, which is given by

$$
L=(1 / 2) m v^{2}+e A \cdot v-e \phi .
$$

The vector $A$ is the vector potential of the magnetic field defined by $B \equiv \nabla \times A$, and the vector $V$ is simply the particle velocity.

The adiabatically conserved magnetic moment, $\mu$ of Eq. (2.27), suggests a possible simpification. The particle kinetic energy perpendicular to the magnetic field,

$$
\mu B \approx(1 / 2) m\left\{v_{\perp}\right\}^{2},
$$

can be treated as a potential energy within the particle Lagrangian. This result is the exact reduction that would be made if the particle gyrophase were entirely ignorable within the Lagrangian. Since the adiabatic conservation of $\mu$ is an extremely accurate approximation 43 for configurations with sufficiently large magnetic field strength, the particle Lagrangian can be approximated in the form first given by Taylor ${ }^{44}$

$$
L=(1 / 2) m\left\{v_{\|}\right\}^{2}+e A \cdot v-\mu B-e \Phi .
$$

${ }^{43}$ Kruskal, M. (1957). Rendiconti del Terzo Congresso Internazionale sui Fenoment D'lonizzazione nei Gas tenuto a Venazia, Societa Italiana di Fisica, Milan, p. 56.

${ }^{44}$ Taylor, J. B. (1964). Phys. Fluids 7, 767. 
The sign of the product $\mu \mathrm{B}$ within Eq. (2.33) may appear erroneous at first, but merely follows from treating $\mu \mathrm{B}$ of $\mathrm{Eq} .(2.32)$ as a potential energy rather than a kinetic energy.

In order to implement Taylor's drift Lagrangian of Eq. (2.33) to derive Boozer's drift Hamiltonian of Eq. (2.26), we must express all variables in terms of Boozer coordinates. A form of the vector potential consistent with the contravariant form of the magnetic field in Eq. (2.12) is

$$
A=\psi \nabla \theta-\chi(\psi) \nabla \varphi
$$

with $X(\psi)$ the poloidal magnetic flux defined in Fig. 2. The contravariant form of the particle velocity is defined by

$$
v=\psi(\partial x / \partial \psi)+\dot{\theta}(\partial x / \partial \theta)+\dot{\varphi}(\partial x / \partial \varphi)
$$

Hence, the inner product $A \cdot V$ becomes

$$
A \cdot v=\psi \dot{\theta}-\chi(\psi) \dot{\varphi}
$$

The covariant form of the magnetic field of Eq. (2.13) and the expression for the particle velocity of Eq. (2.35) yield the component of the particle velocity parallel to the magnetic field,

$$
v_{\|}=\{G(\psi) \dot{\varphi}+I(\psi) \dot{\theta}\} / B(\psi, \theta, \varphi)
$$


The product $\beta_{*} \psi$ was neglected within the equation for $v_{\|}$in Eq. (2.37), because the drift out of a pressure surface, which is determined by $\psi$, is assumed to be much smaller than those given by $\dot{\theta}$ and $\dot{\varphi}$. Using Eqs. (2.36) and (2.37), the expression for the lowestorder drift Lagrangian of Eq. (2.33) becomes

$$
\begin{aligned}
L(\psi, \theta, \varphi, \dot{\theta}, \dot{\varphi})=(1 / 2) m\left\{1 / B^{2}(\psi, \theta, \varphi)\right\}\{G(\psi) \dot{\varphi}+\mathrm{I}(\psi) \dot{\theta}\}^{2} \\
+e\{\psi \dot{\theta}-\chi(\psi) \dot{\varphi}\}-\mu B(\psi, \theta, \varphi)-e \Phi(\psi, \theta, \varphi),
\end{aligned}
$$

in terms of Boozer coordinates.

The Lagrangian of a charged particle is generally a function of six variables: three non-trivial coordinates and their time derivatives. However, the reduced particle Lagrangian of Eq. (2.38) depends explicitly on only five variables, since $\psi$ does not appear. The generalized momenta, $p$, are defined to be the partial derivatives of the Lagrangian with respect to the time derivative of the coordinates, $q$, that is, $p=\partial L / \partial q$. Hence, the vanishing of $\psi$ from the Lagrangian of Eq. (2.38) implies that $\mathrm{P}_{\psi}$ is identically zero. The momenta $P_{\theta}$ and $P_{\varphi}$ agree with that given in Eqs. (2.28) and (2.29).

The Hamiltoian, $H$, is defined in terms of the Lagrangian via

$$
H=\psi \dot{\psi} P_{\psi}+\dot{\theta} P_{\theta}+\dot{\varphi} P_{\varphi}-L
$$

Application of the particle Lagrangian of Eq. (2.38) yields the Boozer 
Hamiltonian of Eq. (2.26). Hamilton's equations are generally given by $\dot{q}=(\partial H / \partial p)$ and $\dot{p}=-(\partial H / \partial q)$, with $q$ a coordinate and $p$ a canonical momentum, as in Eqs. (2.30). Since $p_{\psi}$ vanishes, $\dot{p}_{\psi}=(\partial H / \partial \psi)$ vanishes identically, and $\psi=\left(\partial H / \partial p_{\psi}\right)$ is not well defined. This apparent paradox merely implies that a particle trajectory relative to the $\psi$ surfaces is entirely specified by its values of $\left\{\theta, p_{\theta}, \varphi, p_{\varphi}\right\}$ at some particular time. This result is obvious from the dependence of $\psi$ on the canonical momenta, $p_{\theta}$ and $p_{\varphi}$ given in Eqs. (2.28) and (2.29).

\section{1-D. Isomorphic Equilibria}

The constant pressure surfaces corresponding to axisymmetric tokamak equilibria are simple, nested, axisymmetric tori. The expression for the magnetic field strength corresponding to such equilibria must be independent of the toroidal angle $\varphi$. Thus, the particle drift Hamiltonian of Eq. (2.26) is independent of $\varphi$, so that $P_{\varphi}$ becomes a constant of the motion via Hamilton's equations, Eq. (2.30). This result also applies for the exact particle Hamiltonian corresponding to the exact Lagrangian of Eq. (2.31). This constant of the motion for particle trajectories gives ${ }^{45}$ the well confined transport of energy and particles that are characteristic of axisymmetric tokamak equilibria.

The actual equations governing the transport properties of axisymmetric tokamak equilibria are beyond the scope of this ${ }^{45}$ Boozer, A. H. (1983). Phys. Fluids 26, 496. 
dissertation. However, these equations ${ }^{46}$ are simply expressions for the fluxes of particles, energy, and poloidal flux in terms of the thermodynamic forces which depend upon the profiles of chemical potential, plasma temperature, and toroidal electric current. The matrix elements that relate the fluxes to the thermodynamic forces depend upon the particular collisionality regime of the plasma and the geometry of the confining magnetic field.

Axisymmetric tokamak plasma equilibria exhibit one major drawback, despite their beneficial transport properties. All axisymmetric configurations must possess 47 a toroidal electric current within the plasma, $\mathrm{I}(\psi)$ in Fig. 2 , in order to yield a plasma equilibrium. Hence, some continuous form of current drive is required to maintain any axisymmetric equilibrium. In addition, this toroidal current also provides a source of free energy for plasma instabilities ${ }^{48}$. Non-axisymmetric stellarator configurations do not require continuous current drive to give a plasma equilibrium 49 . However, most stellarator equilibria have extremely poor transport properties due to the lack of an invariant of the canonical momentum type 50 .

Generally, the magnetic field strength within a constant- $\Psi$

${ }^{46}$ Boozer, A. H. (1990). submitted to Phys. Fluids.

${ }^{47}$ Friedberg, J. P. (1987). Ideal Magneto-Hydrodynamics, Plenum Press, New York. p. 107.

${ }^{48}$ Bateman, G. (1978). MHD Instabilities, MIT Press, Cambridge, Massachusetts, and London, England., p. 89.

${ }^{49}$ Friedberg, J. P. (1987). Ideal Magneto-Hydrodynamics, Plenum Press, New York, p. 185.

${ }^{50}$ Beidler, C., et. al. (1990). Fusion Technology: A Journal of the American Nuclear Society 17, 148. 
surface depends on the two angles, $\theta$ and $\varphi$. Suppose that nonaxisymmetric stellarator configurations exist in which the magnetic field strength depends on only the one helical angle $\alpha \equiv \theta-N \varphi$ within the constant- $\Psi$ surfaces, so that it has the form $B(\psi, \theta-N \varphi)$. The corresponding drift Hamiltonian of Eq. (2.26) would also depend only on the helical angle, $\alpha$, within the flux surfaces. The existence of an ignorable coodinate within such a drift Hamiltonian suggests the existence of a canonically conserved momentum analogous to the $P_{\varphi}$ invariant of axisymmetric tokamak equilibria. However, neither of the canonical momenta $P_{\theta}$ and $P_{\varphi}$ of Eqs. (2.28) and (2.29), respectively, are invariant for a magnetic field strength of the form $B(\psi, \theta-N \varphi)$. A change of variable ${ }^{51}$ is obviously required in order to find the desired invariant momentum.

The form of the helical angle $\alpha \equiv \theta-N \varphi$ suggests the replacement $\theta \rightarrow \alpha+N \varphi$ within the two forms of the magnetic field of Eqs. (2.12) and (2.13). Similar forms of the magnetic field are then obtained,

$$
\begin{gathered}
B_{\text {con }}=\nabla \psi \times \nabla \alpha+l_{h}(\psi) \nabla \varphi \times \nabla \psi, \\
B_{\text {cov }}=G_{h}(\psi) \nabla \varphi+I(\psi) \nabla \alpha+\beta_{*}(\psi, \alpha, \varphi) \nabla \psi,
\end{gathered}
$$

with the "helical" rotational transform and poloidal electric current defined by

${ }^{51}$ Boozer, A. H. (1983). Phys. Fluids 26, 496. 


$$
\begin{gathered}
l_{h}(\psi) \equiv l(\psi)-N, \\
G_{h}(\psi) \equiv G(\psi)+N I(\psi),
\end{gathered}
$$

respectively. The new coordinates within these two forms of the magnetic field are $\{\psi, \alpha, \varphi\}$, instead of $\{\psi, \theta, \varphi\}$. These coordinates, $\{\psi, \alpha, \varphi\}$, are guaranteed to be non-trivial since their spatial Jacobian,

$$
J=1 /\{\nabla \psi \cdot\{\nabla \alpha \times \nabla \varphi\}\}=1 /\{\nabla \psi \cdot\{\nabla \theta \times \nabla \varphi\}\}
$$

is identical to that corresponding to the original coordinates, $\{\psi, \theta, \varphi\}$.

The momenta canonically conjugate to the new angular variables, $\{\alpha, \varphi\}$, are given by

$$
\begin{gathered}
P_{\alpha}=\{m \mathrm{I}(\psi) / B(\psi, \theta, \varphi)\} v_{\|}+e \psi, \\
P_{h}=\left\{m G_{h}(\psi) / B(\psi, \theta, \varphi)\right\} v_{\|}-e X_{h}(\psi),
\end{gathered}
$$

with the helical poloidal flux function defined by

$$
X_{h}(\psi) \equiv X(\psi)-N \psi
$$

in analogy with Eqs. (2.28) and (2.29). The new form of the canonical momentum of Eq. (2.46) can be expressed in terms of the old momenta of Eqs. (2.28) and (2.29) via 


$$
P_{h}=P_{\varphi}+N P_{\theta}
$$

This momentum, $p_{h}$, is conserved exactly, within the framework of the guiding-center theory, for all configurations in which the magnetic field strength has the form $B(\psi, \theta-N \varphi)$. For $N=0$, this invariant momentum, $\mathrm{P}_{h}$ of Eq. (2.48), reduces to the $\mathrm{P}_{\varphi}$ invariant corresponding to the $B(\psi, \theta)$ field strength of axisymmetric tokamak equilibria.

Magnetic field strengths corresponding to axisymmetric tokamak equilibria, $\mathrm{B}(\psi, \theta)$, are said to be isomorphic to those of quasi-helically symmetric equilibria, $B(\psi, \theta-N \varphi)$. The equations for isomorphic equilibria examined within this section pertain to field strengths with only one Fourier harmonic, but the results can be generalized52 to apply for field strengths with more than one Fourier harmonic. Since magnetic field strengths with more than one Fourier harmonic do not have an invariant canonical momentum, this dissertation will be restricted to fields with a single Fourier harmonic.

In Chapter VI, we prove that non-axisymmetric configurations which exhibit a magnetic field strength of the form $B(\psi, \theta-N \varphi)$ do not exist. However, we also show that such quasi-helically symmetric equilibria can be well approximated, since the symmetry breaking occurs at third order in a generalized inverse aspect ratio, $\varepsilon \approx 1 / 10$. In addition, the freedom of the magentic field strength 52 Boozer, A. H. (1983). Phys. Fluids 26, 496. 
within one flux surface, which will be developed within Chapter V, permits configurations with exact quasi-helical symmetry on one flux surface. However, the breaking of quasi-helical symmetry near the magnetic axis scales as $\left\{\left(\varepsilon_{a}\right)^{2}-\varepsilon^{2}\right\} \varepsilon$ for a configuration whose flux surface with exact quasi-helical symmetry corresponds to $\varepsilon=\varepsilon_{\mathrm{a}}$.

Nührenberg and Zille 53 have computaionally found threedimensional stellarator equilibria which closely approximate quasihelical symmetry. The gyro-center particle trajectories of such equilibria are very similar to those of axisymmetric tokamak equilibria, in terms of Boozer coordinates. In fact, Monte Carlo particle simulations reveal that these stellarators have either no or a negligible number of super-banana orbits 54 that give the enhanced transport of traditional stellarator configurations.

${ }^{53}$ Nührenberg, J., and Zille, R. (1988). Phys. Lett. A.129, 113.

${ }^{54}$ Beidler, C., et. al. (1990). Fusion Technology: A Journal of the American Nuclear Society 17, 148 


\section{CHAPTER III}

\section{MAGNETIC AXIS}

The magnetic axis is the magnetic field line that corresponds with the central, degenerate plasma pressure surface of a toroidal equilibrium. The only constraint on this magnetic field line is that it must be a smooth, closed curve in three dimensions. The shape and field strength of the magnetic axis largely determine the properties of the neighboring field lines, and thus the magnetic field strength in the vicinity of the axis.

In Chapter IV, we seek solutions to the plasma equilibrium equation, Eq. (1.1), by performing a Taylor expansion in the radial coordinate about a general magnetic axis. To apply such an expansion, a set of unit vectors determined by the magnetic axis itself should be implemented to define the spatial position near the axis. The orthonormal set of Frenet vectors for space curves provides such a convenient vector set.

\section{III-A. Frenet Unit Vectors}

The orthonormal set of Frenet vectors $55\left\{\hat{\kappa}_{0}(l), \hat{\varepsilon}_{0}(l), \hat{b}_{0}(l)\right\}$ (see Fig. 4) define a "locally" Cartesian coordinate system in terms the spatial position, $r_{0}(\ell)$, of any curve. The parameter $\ell$ denotes 55 Mathews, J., and Walker, R. L., (1970). Mathematical Methods of Physics, W. A. Benjamin, Inc., New York, 2nd ed., p. 408. 
the arc length along the curve. The vector $\hat{b}_{0}(l)$ gives the local unit vector that is tangent to the space curve. The unit normal vector $\hat{K}_{0}(l)$ lies in the opposite direction to the local radius of curvature and is perpendicular to $\hat{b}_{0}(l)$. The local unit "binormal" vector $\hat{z}_{0}(l)$ is defined by $\hat{z}_{0}(l) \equiv{\hat{b_{0}}}_{0}(l) \times \hat{\mathbf{k}}_{0}(\ell)$.

The Frenet unit vectors, $\left\{\hat{k}_{0}(l), \hat{\tau}_{0}(l), \hat{b}_{0}(l)\right\}$, are uniquely determined by the spatial position of a curve, $r_{0}(l)$ :

$$
\begin{gathered}
d r_{0} / d l=\hat{b}_{0}(l), \\
d \hat{b}_{0} / d l=k(l) \hat{k}_{0}(l), \\
d \hat{k}_{0} / d l=-k(l) \hat{b}_{0}(l)-\tau(l) \hat{\tau}_{0}(l), \\
d \hat{\tau}_{0} / d l=\tau(l) \hat{k}_{0}(l) .
\end{gathered}
$$

The curvature function $k(l)$ is simply the reciprocal of the local radius of curvature, $R_{c}(l)$, of the curve. The torsion function $\tau(l)$ gives the "twist" of the curve out of a plane.

We now present a derivation of the Frenet vectors, which is found within many mathematics texts 56 . We begin with the spatial position, $r_{0}(l)$, of the curve defined with respect to some coordinate system. We assume that the spatial position is an analytic function of its arc length, $l$, so that all derivatives of $r_{0}(l)$ exist. The differential $d r_{0}(\ell)$ is thus defined, which is clearly tangent to the curve at all points along the curve. The derivative $\mathrm{dr}_{0} / \mathrm{dl}$ yields a vector of unit length that is tangent to the curve at all points. ${ }^{56}$ Mathews, J., and Walker, R. L., (1970). Mathematical Methods of Physics, W. A. Benjamin, Inc., New York, 2nd ed., p. 408. 
Defining the vector $\hat{b}_{0}(l)$ to be this unit tangent to the curve yields Eq. (3.1).

The presumed analyticity of $r_{0}(l)$ implies that the derivative of the unit tangent vector, $\mathrm{db}_{0} / \mathrm{dl}$, is also defined. This vector must be perpendicular to the unit vector $\hat{b}_{0}(l)$, since the derivative of any vector of constant length, but changing direction, is always perpendicular to the original vector. To demonstrate this result, notice that for any vector a of constant length, the equation

$$
0=(d / d t)\left(a^{2}\right)=(d / d t)(a \cdot a)=2 a \cdot(d a / d t)
$$

implies that $(\mathrm{da} / \mathrm{dt})$ must be either exactly zero or perpendicular to the constant vector $a$. The fact that the vector $d \hat{b}_{0} / d \ell$ must be perpendicular to the unit vector $\hat{b}_{0}(l)$ implies that $d \hat{b}_{0} / d l$ can be expressed in the form of Eq. (3.2), with $\hat{b}_{0}(l) \cdot \hat{x}_{0}(l)=0$. The curvature function, $k(l)$ of Eq. (3.2), is the reciprocal of the magnitude of the local radius of curvature, $R_{c}(l)$, of the curve.

The cross product of the two unit vectors $\hat{b}_{0}(\ell)$ and $\hat{k}_{0}(\ell)$ can be used to define a third unit vector

$$
\hat{\tau}_{0}(l) \equiv \hat{b}_{0}(l) \times \hat{K}_{0}(l)
$$

which is mutually perpendicular to the other two. This vector is called the unit "binormal" to curve. Thus, the three orthonormal Frenet vectors, $\left\{\hat{\boldsymbol{k}}_{0}(\ell), \hat{\boldsymbol{\varepsilon}}_{0}(\ell), \hat{b}_{0}(\ell)\right\}$, define a local "Cartesian-like" 
coordinate system that follows the curves and twists of a space curve in three dimensions. For a curve that lies entirely within some plane, the tangent and normal unit vectors, $\hat{b}_{0}(l)$ and $\hat{x}_{0}(l)$, respectively, lie within that plane, whereas the unit binormal, $\hat{\tau}_{0}(\ell)$, is a constant vector perpendicular to the plane.

Differentiating Eq. (3.6) for $\hat{\tau}_{0}(\ell)$ gives

$$
d \hat{z}_{0} / d \ell=\left(d \hat{b}_{0} / d \ell\right) \times \hat{K}_{0}(l)+\hat{b}_{0}(l) \times\left(d \hat{K}_{0} / d \ell\right)=\hat{b}_{0}(l) \times\left(d \hat{K}_{0} / d \ell\right)
$$

with the last step obtained using Eq. (3.2). Hence, $d \hat{\tau}_{0} / d \ell$ is perpendicular to $\hat{b}_{0}(\ell)$, and since $\hat{z}_{0}(l)$ is a unit vector, $d \hat{\tau}_{0} / d \ell$ is also perpendicular to $\hat{\tau}_{0}(\ell)$. Thus, $d \hat{\tau}_{0} / d \ell$ must lie in the direction of $\hat{k}_{0}(l)$, so that it has the general form of Eq. (3.4). The torsion function, $\tau(l)$ of Eq. (3.4), gives the twist of a curve out of a plane. The torsion is exactly zero for a curve that lies entirely within a plane.

The only Frenet equation that has yet to been derived is Eq. (3.3) for $d \hat{K}_{0} / d l$. To derive this equation, differentiate $\hat{K}_{0}(l)=\hat{\tau}_{0}(\ell) \times \hat{b}_{0}(l)$, giving

$$
d \hat{K}_{0} / d l=\left(d \hat{\tau}_{0} / d l\right) \times \hat{b}_{0}(l)+\hat{\tau}_{0}(l) \times\left(d \hat{b}_{0} / d l\right) .
$$

Use of the previously derived Eqs. (3.2) and (3.4) then clearly yields the desired equation for $d \hat{k}_{0} / d l$, Eq. (3.3).

As stated earlier in this section, the magnetic axis of a 
toroidal plasma equilibrium is constrained to be a smooth, closed curve in three dimensions. Hence, the curvature, $k(l)$, and the torsion, $\tau(\ell)$, functions of Eq. (3.1)-(3.4) must be consistent with a smooth, closed curve, in order to give a magnetic axis. Integration of the Frenet equations of Eqs. (3.1)-(3.4) implies that a given $k(l)$ and $\tau(l)$ uniquely determine a space curve to within an irrelevant translation and rotation. The probability that an arbitrary choice of $K(l)$ and $\tau(l)$ yields a smooth, closed curve occupies a set of measure zero. However, sets of $k(l)$ and $\tau(l)$ that give viable magnetic axes can be obtained 57 by implementing a parametric specification of the axis explained within Sec. III-B, or a computational selection of the Fourier harmonics within $k(l)$ and $\tau(\ell)$ developed within Sec. III-C.

\section{III-B. Parametric Specification of the Axis}

One method of finding sets of the curvature, $k(l)$, and the torsion, $\tau(\ell)$, that are consistent with smooth, closed curves is to give ${ }^{58}$ the spatial position, $r_{0}[\ell(\Phi)]$, of a closed curve as a function of some parameter, $\Phi$. An example is given by

$$
r_{0}[\ell(\Phi)]=\{1+\zeta \cos (N \Phi / M)\} \hat{R}(\Phi)+\zeta \sin (N \Phi / M) Z
$$

with $\zeta, N$, and $M$ constants, and $\{\hat{R}(\Phi), \hat{\Phi}(\Phi), \hat{Z}\}$ the standard cylindrical coordinate system. Application of the Frenet equations 57 Garren, D. A., and Boozer, A. H. (1991). Phys. Fluids B: Plasma Physics 3, 2805. ${ }^{58}$ Ibid. 
of Eqs. (3.1)-(3.4) gives the following expressions for the curvature and torsion functions ${ }^{59}$,

$$
\begin{gathered}
\{k(\Phi)\}^{2}=\frac{\left|\left(d r_{0} / d \Phi\right) \times\left(d^{2} r_{0} / d \Phi^{2}\right)\right|^{2}}{\left|\left(d r_{0} / d \Phi\right)\right|^{6}} \\
\tau(\Phi)=\frac{-\left\{\left(d r_{0} / d \Phi\right) \times\left(d^{2} r_{0} / d \Phi^{2}\right)\right\} \cdot\left(d^{3} r_{0} / d \Phi^{3}\right)}{\left|\left(d r_{0} / d \Phi\right) \times\left(d^{2} r_{0} / d \Phi^{2}\right)\right|^{2}}
\end{gathered}
$$

in terms of the axis parameterization, $\Phi$. Expressions for $\left(d r_{0} / d \Phi\right)$, $\left(d^{2} r_{0} / d \phi^{2}\right)$, and $\left(d^{3} r_{0} / d \phi^{3}\right)$ within Eqs. (3.10) and (3.11) are found by using the chain rule of calculus, with the axis length length, $\ell, a$ function of only the parameterization, $\phi$. Substitution of the various derivatives of $r_{0}(\Phi)$ into the right hand side of Eqs. (3.10) and (3.11) readily verifies the equations for the curvature and torsion in terms of $\Phi$. To obtain the curvature and torsion as functions of the axis length, $\ell$, instead of the parameterization, $\Phi$, the differential equation

$$
d \Phi / d \ell=\left\{\left(d r_{0} / d \Phi\right) \cdot\left(d r_{0} / d \Phi\right)\right\}^{-1 / 2}
$$

which holds for any $\Phi$, must be integrated in order to find $\Phi(\ell)$, and thus $K[\phi(\ell)]$ and $\tau[\Phi(\ell)]$.

The $M=1$ family of magnetic axes of Eq. (3.9) corresponds to ${ }^{59}$ Lortz, D., and Nôhrenberg, J. (1979). Z. Naturforsch. 34a, 167. 
conventional $\mathrm{N}$-period, "helical-like" axes. An example with $N=5$, $M=1$, and $C=1 / 15$ is shown in Figs. 1 and 6 . As with most axes of this particular form, the fractional variation of the torsion is larger than that of the curvature.

Various knotted configurations are also possible. Examples are found using Eq. (3.9) with $M=1$. The simplest knot is the $N=3$, $M=2, \zeta=1 / 2$ case shown in Figs. 7 and 8 .

\section{III-C. Computational Optimization of the Axis}

An alternate method for finding sets of the curvature, $k(l)$, and torsion, $r(\ell)$, consistent with closed curves is a computational selection procedure60. As we will prove in Sec. III-D, closed curves can be obtained by varying only two Fourier coefficients within a set of $K(\ell)$ and $\tau(\ell)$. We find closed curves of length $L=2 \pi$ of the form

$$
\begin{aligned}
& k(\ell)=\bar{k}\{1+\delta \cos (2 \pi N \ell / L)\}, \\
& \tau(\ell)=\bar{\tau}\{1-\xi \cos (2 \pi N \ell / L)\},
\end{aligned}
$$

by varying only two of the four parameters $\bar{\kappa}, \bar{\gamma}, \delta$, and $\xi$. For example, suppose that we wish to find a five-period curve of length $L=2 \pi$ with fractional curvature variation $\delta=0.8$ and mean torsion $\bar{\tau} L / 2 \pi=0.25$. The quantity $\bar{\tau} L / 2 \pi$ is of particular interest since it gives the contribution to the lowest order expression for the

${ }^{60}$ Garren, D. A., and Boozer, A. H. (1991). Phys. Fluids B: Plasma Physics 3, 2805. 
FIGURE 6
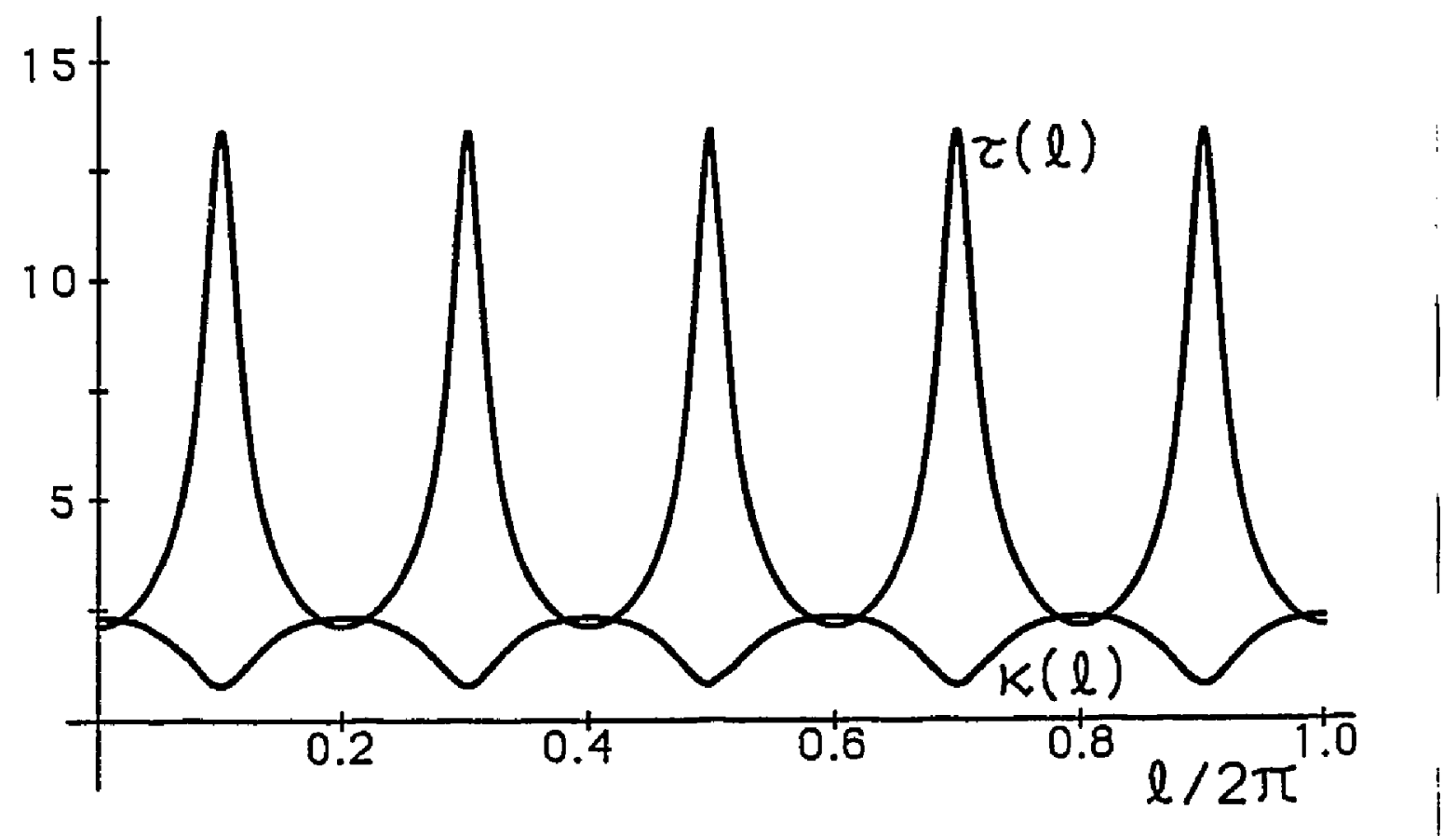

The curvature, $k(l)$, and torsion, $\tau(l)$, functions corresponding to the "helical-like" axis of Fig. 1 with axis length $2 \pi$. 
FIGURE 7

THREE PERIOD "KNOTTED" AXIS

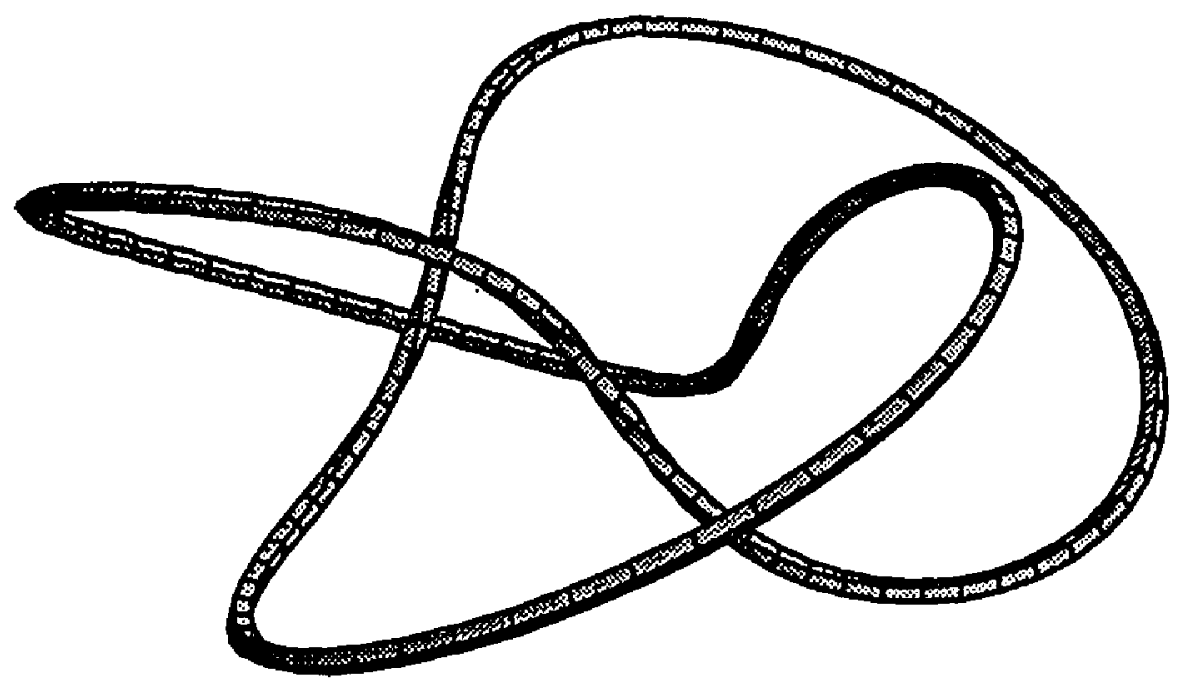

A "knotted" axis given by Eq. (3.9) with $M=2, N=3$, and $\zeta=1 / 2$. 


\section{FIGURE 8}

\section{CURVATURE AND TORSION OF THREE PERIOD KNOTTED AXIS}

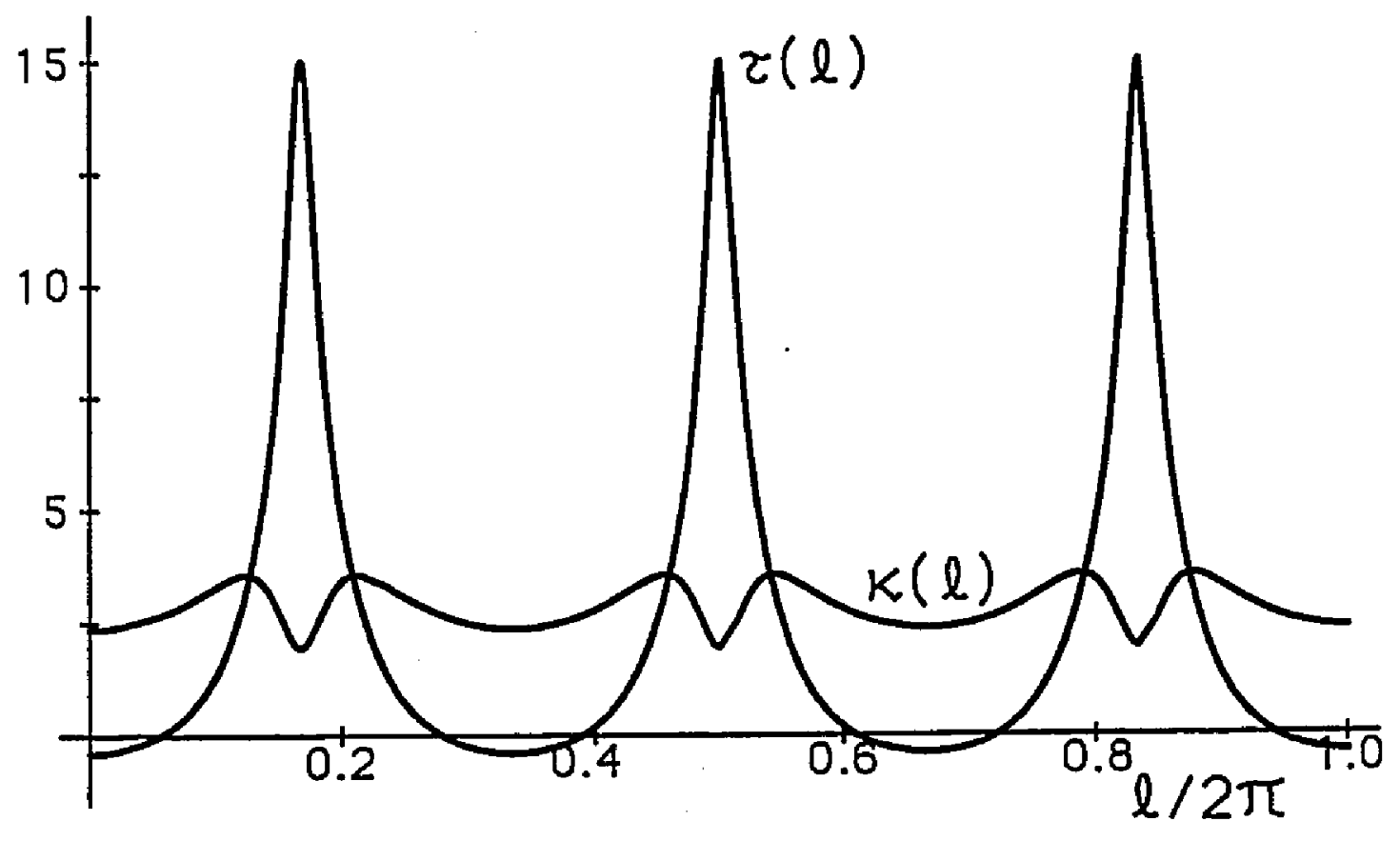

The curvature, $k(\ell)$, and torsion, $\tau(\ell)$, functions corresponding to the "knotted" axis of Fig. 7 with length $L=2 \pi$. 
rotational transform, $2(\psi)$ of Fig. 2 , due to torsion. The parameters $\bar{K}$ and $\xi$ are varied until the integration of the Frenet formulae, Eqs. (3.1)-(3.4), yields a smooth, closed curve. The curve obtained depends on the choice of the initial values of the parameters $\bar{k}$ and $\xi$. One solution is $\bar{K} L / 2 \pi=1.741$ and $\xi=26.36$; a graph of this curve is shown in Fig. 9.

We can optimize the magnetic axis shape under some additional restriction. For example, we may wish to minimize the maximum curvature along the length of an axis with a given mean torsion. Assuming the form of the curve given in Eqs. (3.13) and (3.14) with $\bar{\tau} L / 2 \pi=0.5$, our optimization routine leads to a "knotted" curve with parameters $\bar{K} L / 2 \pi=3.337, \delta=0.531$, and $\zeta=4.779$, which is shown in Fig. 10. A curve optimized to have constant curvature is shown in Fig. 11. The parameters of this "helical-like" curve are given by $\bar{K} L / 2 \pi=4.746, \delta=0.0$, $\bar{\tau} L / 2 \pi=2.0$, and $\zeta=1.070$.

\section{III-D. Proof of Two Parameter Optimizations}

In this section, we demonstrate that only two free parameters are required 61 in order that integration of the Frenet formulae of Eqs. (3.1)-(3.4) produce a smooth, closed curve. This analysis justifies the use of only two variable parameters in the construction of magnetic axes developed within the previous section.

${ }^{61}$ Garren, D. A., and Boozer, A. H. (1991). Phys. Fluids B: Plasma Physics 3, 2805. 
FIGURE 9

\section{COMPUTATIONALLY SELECTED AXIS}

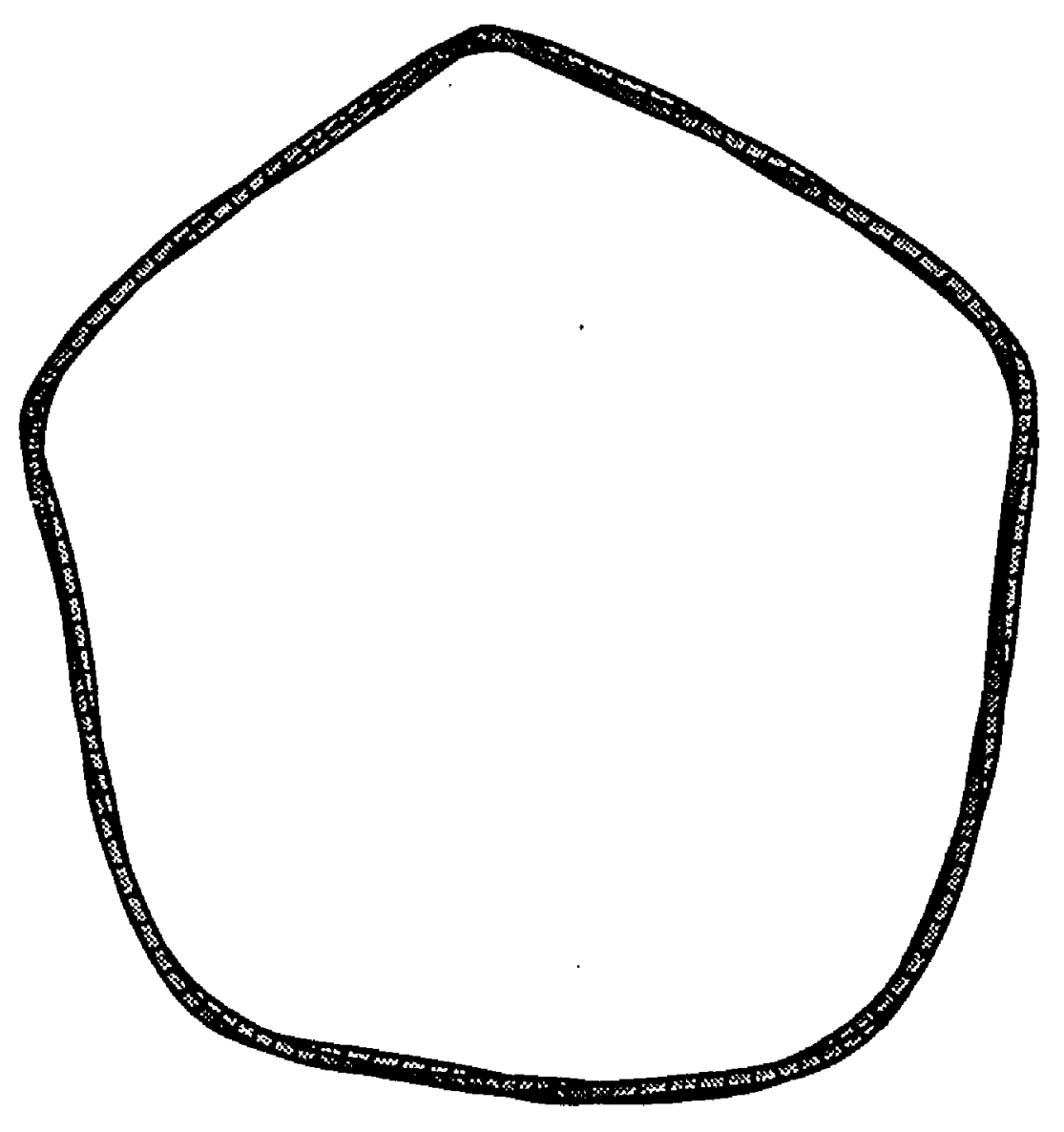

A curve of the form of Eqs. (3.13) and (3.14) with $\bar{k} L / 2 \pi=1.741$, $\delta=0.8, \bar{\tau} L / 2 \pi=0.25$, and $\xi=26.36$ obtained by computationally selecting the values of $\bar{k}$ and $\xi$. 
FIGURE 10

\section{AXIS WITH OPTIMIZED MAXIMUM CURVATURE}

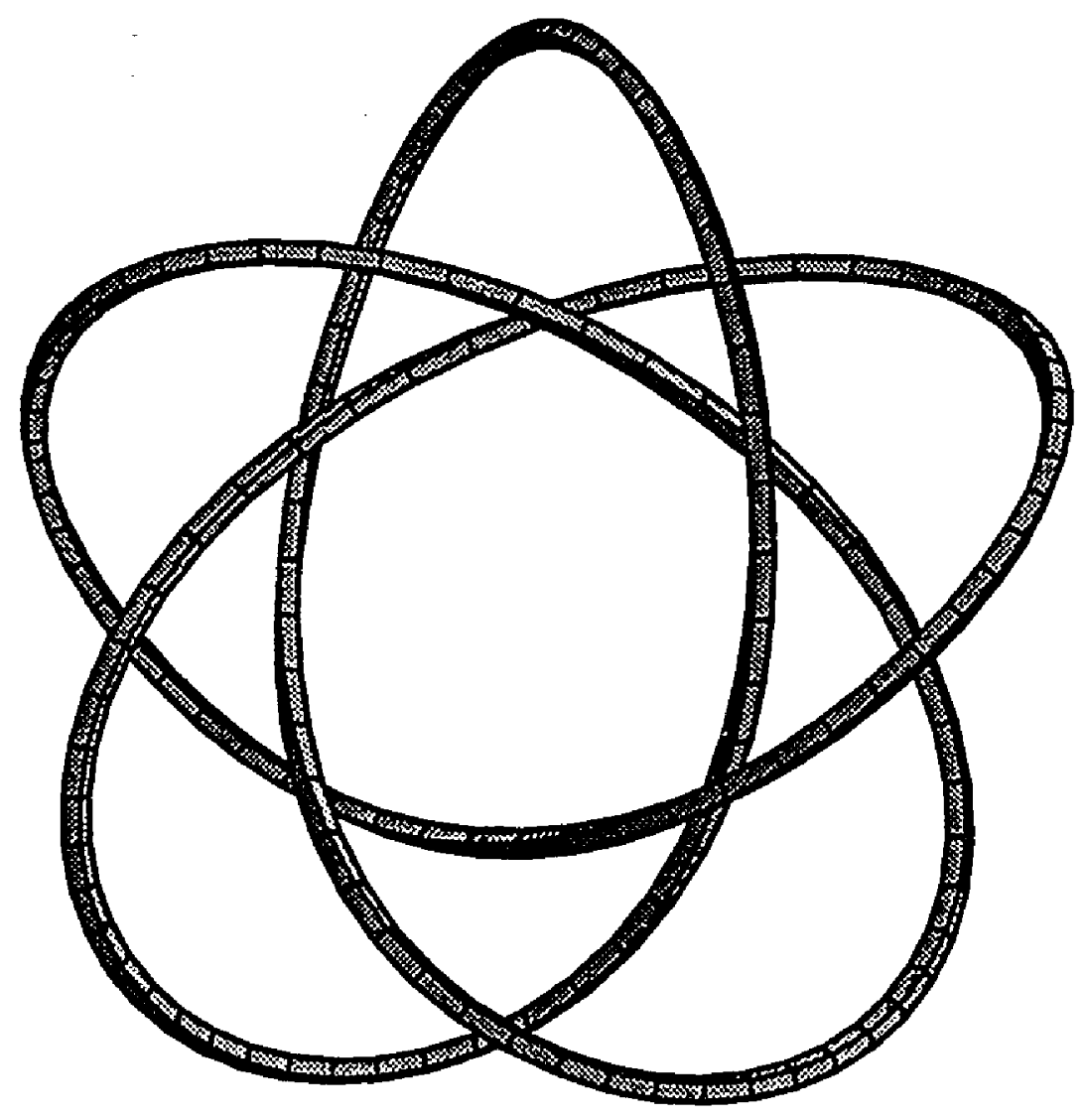

A curve of the form of Eqs. (3.13) and (3.14) with $\bar{K} L / 2 \pi=3.337$, $\delta=0.531, \bar{\tau} L / 2 \pi=0.5$, and $\xi=4.779$ obtained by optimizing $\bar{k}, \delta$, and $\xi$ to find a curve with a given mean torsion $\bar{\tau} L / 2 \pi$ and $a$ minimum value for the maximum curvature along its length. 


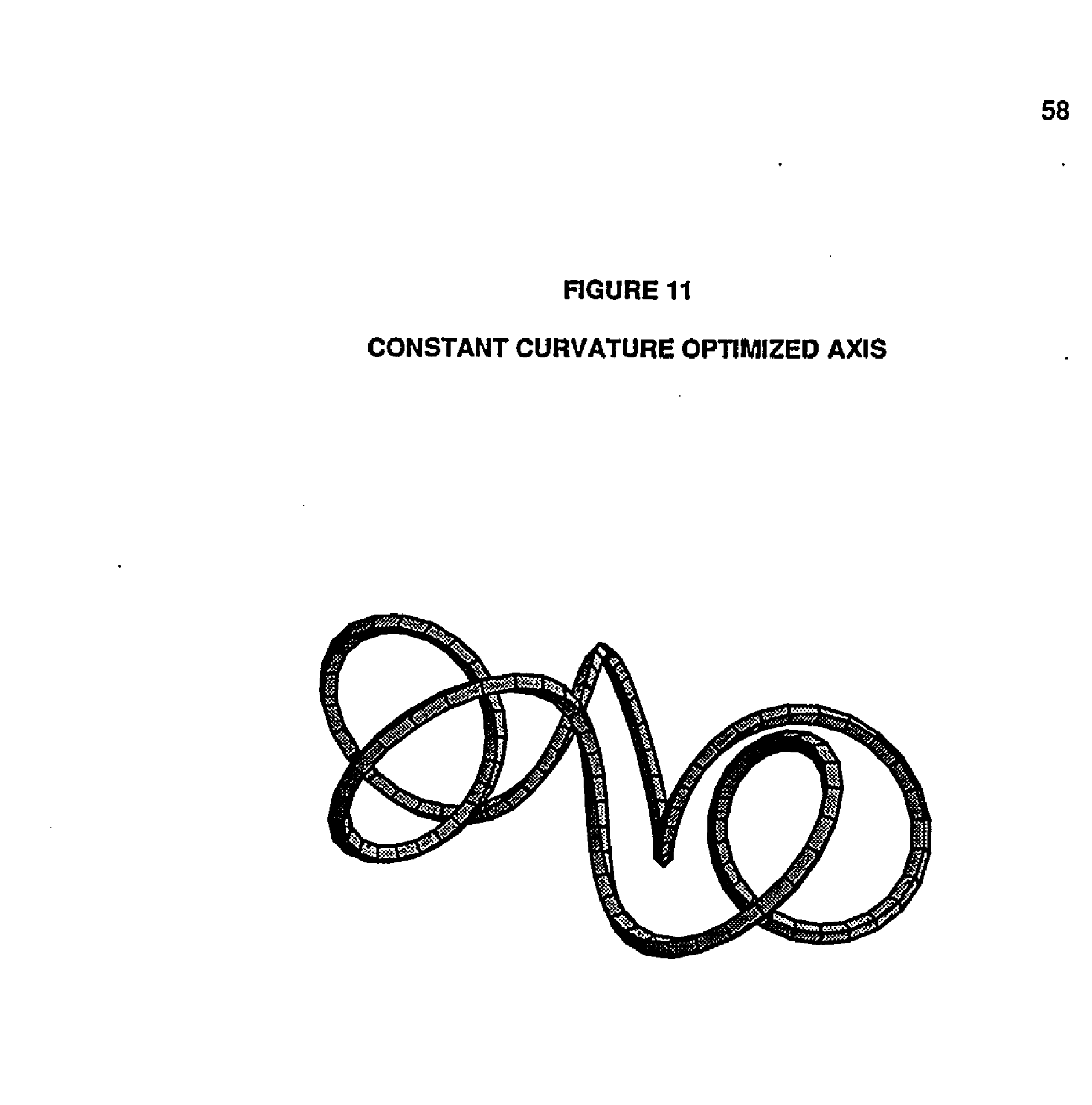

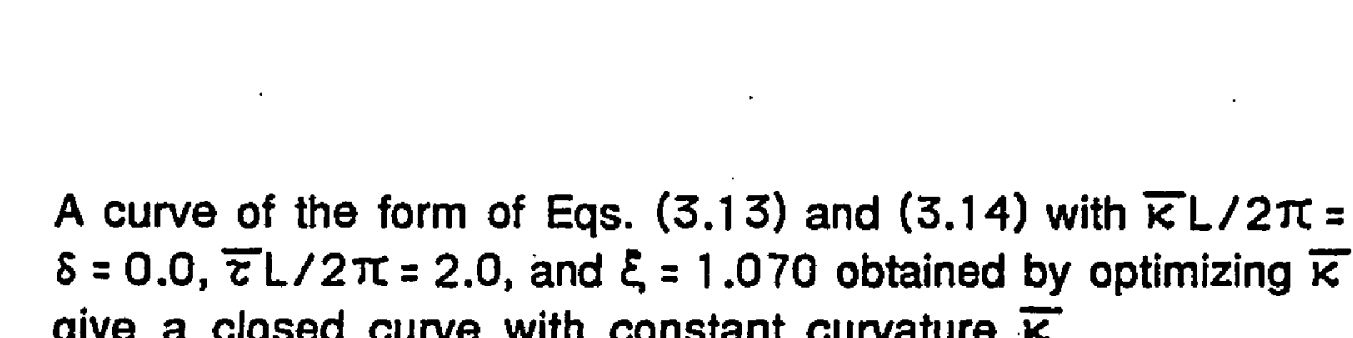


We assume analytic forms of the curvature and torsion functions, as in Eqs. (3.13) and (3.14), with two variable Fourier coefficients within this set for the selection procedure to give a smooth, closed curve. We assume that these functions have some non-trivial periodicity other than that corresponding to the length of the curve itself. That is, both the curvature, $k(\varphi)$, and torsion, $\tau(\varphi)$, must both have period $2 \pi / N$, with $N=1$, if the period of $\varphi$ is $2 \pi$. Then, given an initial set of Frenet vectors defined at a point in space, the Frenet equations of Eqs. (3.1)-(3.4) are integrated over its lowest period, $2 \pi / \mathrm{N}$, giving a new set of Frenet vectors.

The Frenet vectors corresponding to any point in the integration of Eqs. (3.1)-(3.4) always form an orthonormal set 62 . Thus, the set of Frenet unit vectors can be treated as a rigid body in three dimensions. The general transformation of any rigid body is a rotation followed by a translation. Hence, the integration of the Frenet vectors over one period can treated as a general transformation of the rigid set of initial Frenet vectors. That is, the Frenet vectors after integration through one period of the curvature and torsion functions can be obtained by a rotation followed by a translation of the initial set of Frenet vectors. We apply the active view of transformations in which the coordinate system is held fixed once it is chosen. Therefore, all transformations are actually applied to the position and orientation of the Frenet vectors themselves.

62Mathews, J., and Walker, R. L., (1970). Mathematical Methods of Physics, W. A. Benjamin, Inc., New York, 2nd ed., p. 408. 
We now find constraints on the form of general transformations of a set of Frenet vectors, which applied $N$ times, return the vectors to their initial position and orientation. The general tranformation $\mathcal{T}$ of any such rigid body is a rotation $R$ followed by a displacement d, i.e.,

$$
\mathcal{T} x=R+d,
$$

with the transpose of the column vector $x$ given by $(x, y, z)$. The vector $X$ gives the coordinates corresponding to any point in space. We seek forms of the transformation of Eq. (3.15) such that $N$ applications give the original coordinates of all points in space, i.e.,

$$
\tau^{N} x=x
$$

Such a desired result corresponds to integration of the Frenet formulae over $N$ periods giving a curve which joins its initial position smoothly. Use of Eq. (3.15) within Eq. (3.16) thus implies

$$
R^{N} X+\left\{\sum_{j=0}^{N-1} R^{j}\right\} d=x,
$$

must hold for any position vector $x$. This result follows if the equations 


$$
\begin{gathered}
R^{N} X=X, \\
\left\{\sum_{j=0}^{N-1}\right\} d=0
\end{gathered}
$$

both hold. We will now show that only one constraint must be applied to the form of the rotation matrix, $R$, and only one constraint to the form of the displacement vector, $d$, in order to satisfy (3.18) and (3.19) with $N=1$.

The axis about which to perform the rotation, $R$, is entirely arbitrary. However, we will show that the angle of rotation, $\alpha$, about this axis is constrained to be a rational number in order to satisfy Eq. (3.18). To prove this result, choose the z-axis of our Cartesian coordinate system to correspond with the axis of rotation. The choice of the $x$ and $y$ axes is arbitrary. Using the standard rotation matrix corresponding to this coordinate system,

$$
R(\alpha)=\left[\begin{array}{ccc}
\cos (\alpha) & \sin (\alpha) & 0 \\
-\sin (\alpha) & \cos (\alpha) & 0 \\
0 & 0 & 1
\end{array}\right]
$$

the constraint of Eq. (3.18) implies that the rotation angle, $\alpha$, must be $2 \pi M / N$, with $M$ any integer.

We now use $\alpha=2 \pi M / N$ within $R$ in Eq. (3.19) to determine any additional constraints. Notice that multiple application of the rotation matrix gives $R j(\alpha)=R(j \alpha)$, which is obvious using Eq. 
(3.20). Thus, the sum of the rotation matrices within Eq. (3.19) gives a finite sum of the form $\sum \cos (j 2 \pi M / N)$ for its $x x$ and $y y$ components, and one of the form $\pm \sum \sin (j 2 \pi M / N)$ for its $x y$ and $y x$ components. Application of the relations

$$
\begin{gathered}
\cos (\alpha)=\{1 / 2\}\{\exp (i \alpha)+\exp (-i \alpha)\}, \\
\sin (\alpha)=\{1 /\{2 i\}\}\{\exp (i \alpha)-\exp (-i \alpha)\}
\end{gathered}
$$

then gives finite geometric series of the form

$$
\sum_{j=1}^{N} r^{j}=\frac{1-r^{N}}{1-r}
$$

for each of the $x x, x y, y x$, and $y y$ elements of the matrix sum of Eq. (3.19). Since the value for $r$ in each of these series is $\exp ( \pm 2 \pi i M / N)$, these matrix elements all sum to zero, provided $N=1$. The geometric series of Eq. (3.23) is indeterminant for $N=1$. The only non-zero element within the resulting matrix of Eq. (3.19) is the $z z$ element, which trivially reduces to $N$.

The transpose of the displacement vector, $d$, takes the general form $\left(x_{0}, y_{0}, z_{0}\right)$. Application of the sum of matrices within Eq. (3.19) then simply gives

$$
\left(0,0, N z_{0}\right)=(0,0,0)
$$


This equation shows that the displacement parallel to the axis of rotation, which is given by $z_{0}$, is constrained to be zero. The displacement perpendicular to the axis of rotation, which is determined by $x_{0}$ and $y_{0}$, is arbitrary.

We have shown that in order satisfy Eq. (3.16), the rotation about the arbitrary axis is constrained to be a rational angle and the displacement that follows is constrained to be zero parallel to the axis of rotation, provided $N=1$. Thus, there are only two constraints that must be satisfied in finding a general transformation of the set of Frenet vectors which, applied some finite number of times, is equivalent to the identity transformation. Therefore, the two variable parameters within the curvature and torsion functions are sufficient to yield a smooth, closed curve, provided that the curvature and torsion functions both have some non-trivial periodicity. 


\section{CHAPTER IV}

\section{TOROIDAL PLASMA EQUILIBRIA}

In this chapter, a generic method for finding threedimensional, toroidal plasma equilibria is developed. These equilibria are solutions to the plasma equilibrium equation, $\mathrm{j} \times \mathrm{B}=\nabla p$, with $\mathrm{j}=\left(1 / \mu_{0}\right) \nabla \times B$, via Ampere's law, coupled with the divergence-free property of the magnetic field, $\nabla \cdot B=0$. Plasma equilibria are actually constructed by performing a Taylor series expansion about a general magnetic axis. Comprehension of this expansion methodology is vital to the elucidation of the restrictions in the form of the magnetic field strength that are given within Chapter V.

\section{IV-A. Basic Equations of Toroidal Plasma Equilibria}

In Sec. II-B, we presented a derivation of a set of curvilinear coordinates, $\{\psi, \theta, \varphi\}$, (see Fig. 2), developed by Boozer63. The constant surfaces of the radial coordinate, $\psi$, correspond to the nested, toroidal, plasma pressure surfaces. The poloidal angle, $\theta$, defines the "short way" around the torus and the toroidal angle, $\varphi$, defines the "long way" around the torus. The primary advantage of implementing these coordinates is that they are the canonical ${ }^{63}$ Boozer, A. H. (1981). Phys Fluids 24, 1999. 
coordinates of the guiding-center drift Hamiltonian presented within Sec. II-C. Furthermore, widely differing plasma equilibria with related magnetic field strengths in terms of Boozer coordinates can have similar 64 transport properties, as outlined within Sec. II-D.

The use of Boozer coordinates permits the magnetic field of any toroidal plasma equilibrium to be expressed simultaneously in the two simplified representations ${ }^{65}$ of Eqs. (2.12) and (2.13). The only critical assumption used is that the magnetic field can never vanish within the toroidal region. The contravariant form of the magnetic field of Eq. (2.12), which is divergence free, insures that the magnetic field lines lie within surfaces of constant pressure. The covariant form of the field in Eq. $(2.13)$ guarantees that the electric current trajectories lie within these same pressure surfaces. The constraint that the field lines and current trajectories lie within pressure surfaces is given by Eq. (1.2), which trivially follows from the plasma equilibrium equation of Eq. (1.1).

The equality of the two representations of the magnetic field of Eqs. (2.12) and (2.13) implies the constraint 66

$$
\nabla \psi \times \nabla \theta+L(\psi) \nabla \varphi \times \nabla \psi=G(\psi) \nabla \varphi+I(\psi) \nabla \theta+\beta_{*}(\psi, \theta, \varphi) \nabla \psi,
$$

for all scalar-pressure, equilibrium magnetic fields expressed in ${ }^{64}$ Boozer, A. H. (1983). Phys. Fluids 26, 496.

${ }^{65}$ Boozer, A. H. (1981). Phys Fluids 24, 1999.

${ }^{66}$ Garren, D. A., and Boozer, A. H. (1991). Phys. Fluids B: Plasma Physics 3, 2805. 
terms of Boozer coordinates. The constraint of Eq. (4.1) guarantees that the magnetic field is divergence-free, and that both the magnetic field lines and electric current trajectories lie within the toroidal plasma pressure surfaces. Application of the dual representation of the magnetic field via Eq. (4.1) also reduces the vector plasma equilibrium equation of Eq. (1.1), $\mathrm{j} \times \mathrm{B}=\nabla \mathrm{p}$, to a scalar equation, as will now be given.

The plasma equilibrium equation of Eq. (1.1) can be expressed in the form

$$
\left\{\nabla \times B_{\text {cov }}\right\} \times B_{\text {con }}=\mu_{0} \nabla p,
$$

using Ampere's law, $\nabla \times B=\mu_{0} j$, and the two forms of the magnetic field of Eqs. (2.12) and (2.13). This vector equation condenses to the scalar equation

$$
\begin{aligned}
\{(d G / \partial \psi)- & \left.\left(\partial \beta_{*} / \partial \varphi\right)\right\}+2(\psi)\left\{(d l / \partial \psi)-\left(\partial \beta_{*} / \partial \theta\right)\right\} \\
& +\mu_{0}(d p / \partial \psi)\{G(\psi)+2(\psi) I(\psi)\} / B^{2}(\psi, \theta, \varphi)=0,
\end{aligned}
$$

since both the $\nabla \theta$ and $\nabla \varphi$ components of Eq. (4.2) reduce to zero. The scalar force balance equation of Eq. (4.3) provides an additional constraint on equilibria with non-trivial current and pressure profiles. In deriving Eq. (4.3), the spatial Jacobian of the Boozer coordinates given in Eq. (2.25) was implemented.

The scalar force balance equation of Eq. (4.3) can be solved 
analytically if both $\beta_{*}(\psi, \theta, \varphi)$ and $1 / B^{2}(\psi, \theta, \varphi)$ are fully Fourier decomposed in both $\theta$ and $\varphi$. Therefore, $1 / B^{2}$ should be expressed in the form 67

$$
\begin{aligned}
1 / B^{2}(\psi, \theta, \varphi)=\left\{1 /\left\langle B^{2}\right\rangle\right\}\left\{1+\sum^{\prime}\left[\gamma_{n, m}(\psi) \cos (n \varphi-m \theta)\right.\right. \\
\left.\left.+\lambda_{n, m}(\psi) \sin (n \varphi-m \theta)\right]\right\}
\end{aligned}
$$

with the apostrophe on the summation indicating the omission of the $n=0, m=0$ term. The flux function $\left\langle B^{2}\right\rangle$ of Eq. (4.4) is the average of $B^{2}(\psi, \Theta, \varphi)$ within a magnetic surface, i.e.,

$$
\left\langle B^{2}\right\rangle \equiv \frac{(\partial / \partial \psi) \int B^{2} \partial^{3} x}{(\partial / \partial \psi) \int d^{3} x}
$$

To obtain Eq. (4.5), the spatial Jacobian of the Boozer coordinates, Eq. (2.25), and the relation $d^{3} x=J d \psi d \theta d \varphi$ for transforming volume elements were both utilized.

Averaging the scalar equilibrium equation of Eq. (4.3) over $\theta$ and $\varphi$ gives $^{68}$

$$
d G / d \psi+l(\psi) d l / d \psi+\mu_{0}(d p / d \psi)\left\{[G(\psi)+2(\psi) I(\psi)] /\left\langle B^{2}\right\rangle\right\}=0
$$

This procedure then implies that the function $\beta_{*}(\psi, \theta, \varphi)$ has the form

67 Boozer, A. H. (1981). Phys Fluids 24, 1999.

${ }^{68}$ Kruskal, M. D., and Kulsrud, R. M. (1958). Phys. Fluids 1, 265. 


$$
\begin{aligned}
\beta_{*}(\psi, \theta, \varphi)= & \mu_{0}(d p / d \psi)\left\{\{G(\psi)+l(\psi) I(\psi)\} /\left\langle B^{2}\right\rangle\right\} \\
& \times \Sigma^{\prime}\{1 /[n-L(\psi) m]\}\left\{\gamma_{n, m}(\psi) \sin (n \varphi-m \theta)\right. \\
& \left.-\lambda_{n, m}(\psi) \cos (n \varphi-m \theta)\right\},
\end{aligned}
$$

with the functions $\gamma_{n, m}(\psi)$ and $\alpha_{n, m}(\psi)$ determined by the Fourier coefficients of $1 / B^{2}$ in Eq. (4.4). The additional function of $\psi$ that may be added to $\beta_{*}(\psi, \theta, \varphi)$ can be absorbed into the choice of the toroidal angle, $\varphi$, by a transformation of the form $\varphi \rightarrow \varphi+f(\psi)$. The function $1 / B^{2}$ is assumed to be analytic, so that the functions $\gamma_{n, m}(\psi)$ and $\lambda_{n, m}(\psi)$ of Eqs. (4.4) and (4.7) have the form

$$
\delta_{n, m}(\psi)=\psi^{m / 2} h(\psi)
$$

with $h(\psi)$ some analytic function of $\psi$ and $m$ some nonnegative integer. The generic form of analytic functions in terms of Boozer coordinates will be presented within Sec. IV-C.

In summary, the vector differential equation resulting from the equality of the two forms of the magnetic field, Eq. (4.1), and the scalar equilibrium equation of Eq. (4.3) define a scalar-pressure, MHD equilibrium in terms of Boozer coordinates. The constants of integration associated with the differential equation of Eq. (4.1) are sufficient to give a continuous spectrum of MHD equilibria and are equivalent to freedom of the shape of the bounding magnetic flux surface.

The scalar force balance equation of Eq. (4.3) is satisfied if 
the average force balance equation, Eq. (4.6), holds with the function $\beta_{*}(\psi, \theta, \varphi)$ given by Eq. (4.7), which involves the Fourier coefficients of the magnetic field strength. Hence, the equations that define scalar-pressure equilibria can be given by Eqs. (4.1), (4.6), and (4.7). These constraint equations prevent arbitrary specification of the magnetic field strength in terms of Boozer coordinates. Thus, magnetic field strengths that give desirable guiding-center drift trajectories and associated transport may not correspond to actual toroidal plasma equilibria.

An annotation is made for plasma equilibria with more than one field period. All periodic equilibria are mathematically equivalent to those with only one field period. This result is demonstrated by the transformation 69

$$
\begin{aligned}
& N \varphi \rightarrow \varphi, \\
& L \rightarrow N L, \\
& L \rightarrow N \&, \\
& G \rightarrow N G,
\end{aligned}
$$

with $Q, R, 8$, and (5) the per-period quantities of the toroidal angle, magnetic axis length, rotational transform, and poloidal electric current, respectively. The form of the resulting equations in terms of the new variables is identical to that of the old, without the period, N, appearing explicitly.

${ }^{69}$ Garren, D. A., and Boozer, A. H. (1991). Phys. Fluids B: Plasma Physics 3, 2805. 


\section{IV-B. Inverse Method for Defining the Coordinates}

Finding sets of $\{\psi, \theta, \varphi, G(\psi), I(\psi), l(\psi), p(\psi)\}$ which satisfy the equilibrium equations of Eqs. (4.1), (4.6), and (4.7) is quite arduous. We seek solutions by performing an Taylor series expansion about an arbitrary magnetic axis, since the most critical constraints of MHD equilibria are determined by the lowest order terms in the expansion. To implement this expansion technique, the basic equations are expressed in terms of the spatial position $\times(\psi, \theta, \varphi)$ (see Fig. 3), instead of the coordinates, $\{\psi(x), \theta(x), \varphi(x)\}$. This inverse transformation is accomplished through the use of the dual relations 70 of partial differential theory, which were presented in Sec. II-A. Application of the dual relations to Boozer's coordinates, $\{\psi, \theta, \varphi\}$, yields

$$
\partial x / \partial \psi=J \nabla \theta \times \nabla \varphi \text { and }(\partial x / \partial \theta) \times(\partial x / \partial \varphi)=J \nabla \psi \text {, }
$$

plus all even permutations of $\{\psi, \theta, \varphi\}$, with the spatial Jacobian $J$ given by

$$
J=\{(\partial x / \partial \psi) \times(\partial x / \partial \theta)\} \cdot(\partial x / \partial \varphi)=1 /\{\{\nabla \psi \times \nabla \theta\} \cdot \nabla \varphi\}
$$

Using the dual relations of Eqs. (4.13) and (4.14), the equality of the two forms of the magnetic field via Eq. (4.1) becomes the vector differential equation 71

70White, R. B. (1989). Theory of Tokamak Plasmas, North Holland Physics, Amsterdam, p. 6.

${ }^{71}$ Garren, D. A., and Boozer, A. H. (1991). Phys. Fluids B: Plasma Physics 3, 2805. 


$$
\begin{aligned}
(\partial x / \partial \varphi)+L(\psi)(\partial x / \partial \theta)=G & (\psi)(\partial x / \partial \psi) \times(\partial x / \partial \theta) \\
& +I(\psi)(\partial x / \partial \varphi) \times(\partial x / \partial \psi) \\
& +\beta_{*}(\psi, \theta, \varphi)(\partial x / \partial \theta) \times(\partial x / \partial \varphi) .
\end{aligned}
$$

The constraint of Eq. (4.15) is expressed in terms of partial derivatives of the spatial position, $x(\psi, \theta, \varphi)$, whereas the original equation of Eq. (4.1) uses the gradients of the coordinates, $\{\psi(x), \theta(x), \varphi(x)\}$. Toroidal plasma equilibria can be defined using Eqs. (4.15), (4.6), and (4.7), in lieu of Eqs. (4.1), (4.6), and (4.7). We implement Eq. (4.15) throughout the remainder of this dissertation, since this equation applies the inverse method for defining the coordinates, which facilitates the expansion about the axis.

The Jacobian of the Boozer coordinates, given by Eqs. (2.25) and (4.14) permit the magnetic field strength to be expressed in the form

$$
\frac{1}{B^{2}(\psi, \theta, \varphi)}=\frac{(\partial x / \partial \psi) \cdot\{(\partial x / \partial \theta) x(\partial x / \partial \varphi)\}}{G(\psi)+l(\psi)](\psi)}
$$

However, a form that will prove to be more enlightening within Sec. IV-(E-G) is obtained by applying the inner product of the contravariant form of the magnetic field, Eq. (2.12), with itself. This procedure yields 


$$
\frac{1}{B^{2}(\psi, \theta, \varphi)}=\frac{\|(\partial x / \partial \varphi)+L(\psi)(\partial x / \partial \theta)\|^{2}}{\{G(\psi)+L(\psi) !(\psi)\}^{2}}
$$

using the form of $1 / B^{2}$ in Eq. (4.16).

We perform the Taylor expansion about a general magnetic axis using the orthonormal set of Frenet vectors $72,\left\{\hat{k}_{0}(l), \hat{z}_{0}(l), \hat{b}_{0}(l)\right\}$ (see Fig. 4), defined within Sec. III-A. The vector $\vec{b}_{0}(l)$ is simply the local unit vector that is tangent to a space curve, everywhere. The unit normal vector $\hat{K}_{0}(l)$ lies in the opposite direction of the local radius of curvature, and the unit binormal vector $\hat{\zeta}_{0}(\ell)$ is mutually perpendicular to the other two.

These Frenet unit vectors form a local "Cartesian-like" coordinate system with repect to any smooth curve. Therefore, these vectors can be used to express the spatial position near the axis in the form ${ }^{73}$

$$
\begin{aligned}
X(\psi, \theta, \varphi)=r_{0}[\ell(\varphi)]+X(\psi, \theta, \varphi) \hat{k}_{0}[\ell(\varphi)]+ & Y(\psi, \theta, \varphi) \hat{z}_{0}[\ell(\varphi)] \\
& +Z(\psi, \theta, \varphi) \hat{b}_{0}[\ell(\varphi)] .
\end{aligned}
$$

We constrain $X(\psi=0)=Y(\psi=0)=Z(\psi=0)=0$, so that the vector function $r_{0}[\ell(\varphi)]$ corresponds to the magnetic axis, with $\ell(\varphi)$ the distance along the axis. The functions $\{X(\psi, \theta, \varphi), Y(\psi, \theta, \varphi), Z(\psi, \theta, \varphi)\}$ of the spatial position of Eq. (4.18) can be used to express an 72 Mathews, J., and Walker, R. L., (1970). Mathematical Methods of Phys/cs, W. A. Benjamin, Inc., New York, 2nd ed., p. 408.

${ }^{73}$ Garren, D. A., and Boozer, A. H. (1991). Phys. Fluids B: Plasma Physics 3, 2805. 
arbitrary point near the magnetic axis in terms of the coordinates $\{\psi, \theta, \varphi\}$. The form of the spatial position in Eq. (4.18) is completely general, since the corresponding Frenet Jacobian,

$$
J_{F}=1 /\{\{\nabla X \times \nabla Y\} \cdot \nabla Z\},
$$

is neither zero, nor infinite, in the region of interest.

The form of the spatial position given in Eq. (4.18) is not unique. For example, an alternate representation is given by

$$
x(\psi, \theta, \varphi)=Q(\psi, \theta, \varphi) \hat{x}+R(\psi, \theta, \varphi) \hat{y}+S(\psi, \theta, \varphi) \hat{z}
$$

with $\{\hat{x}, \hat{y}, \hat{z}\}$ the Cartesian unit vectors. For a given equilibrium, the form of the functions $\{Q(\psi, \theta, \varphi), R(\psi, \theta, \varphi), S(\psi, \theta, \varphi)\}$ of Eq. (4.20), is changed under a coordinate translation or rotation. However, the form of the functions $\{X(\psi, \theta, \varphi), Y(\psi, \theta, \varphi), Z(\psi, \theta, \varphi)\}$ of the Frenet representation of Eq. (4.18) is independent of the coordinate origin and orientation. Hence, the Frenet representation does not contain any apparent freedom in the configuration that is merely due to the trivial freedoms of translations and rotations of the coordinate system.

We employ the Frenet representation of the spatial position in Eq. (4.18) throughout the remainder of this dissertation. This use of the Frenet representation is essential to the counting of the free functions of $\varphi$ that will be developed within Chapter $V$ on the 
restrictions of the magnetic field strength. Use of a representation other than Eq. (4.18) may give additional free functions of $\varphi$ that represent irrelevant translations or rotations of the coordinate system, rather than any additional freedom in the plasma equilibrium.

\section{IV-C. Analytic Functions of Boozer Coordinates}

We have shown that the task of finding toroidal plasma equilibria can be defined using Eqs. (4.15), (4.6), and (4.7). Generic solutions will be found in the remainder of this chapter by performing a Taylor series expansion about a general magnetic axis. To comprehend the details of this expansion technique, the general form of an analytic function in terms of Boozer coordinates must first be understood.

We will demonstrate that any function that is analytic in some local toroidal region can be cast in the form

$$
f(\psi, \theta, \varphi)=\sum_{m=0}^{\infty} \psi^{m / 2}\left\{a_{m}(\psi, \varphi) \sin (m \theta)+b_{m}(\psi, \varphi) \cos (m \theta)\right\}, \quad \text { (4.21) }
$$

in terms of Boozer coordinates, $\{\psi, \theta, \varphi)$. The poloidal coefficients $a_{m}(\psi, \varphi)$ and $b_{m}(\psi, \varphi)$ are analytic functions of $\psi$ and $\varphi$, so that they are expressed as

$$
a_{m}(\psi, \varphi)=a_{m, 0}(\varphi)+a_{m, 2}(\varphi) \psi+a_{m, 4}(\varphi) \psi^{2}+\cdots,
$$


with $a_{m, 0}(\varphi), a_{m, 2}(\varphi), a_{m, 4}(\varphi)$, etc., periodic in $\varphi$.

To begin the proof of Eqs. (4.21) and (4.22), assume that $\xi(x)$ and $\eta(x)$ define "Cartesian-like" coordinates such that $\nabla \xi$ and $\nabla \eta$ are finite and non-zero within some two-dimensional surface of interest. If the cross product $\nabla \xi \times \nabla \eta$ is nonzero within the surface, then any analytic function of position can be expanded in a power series of the form 74

$$
f(\varepsilon, \eta)=\sum f_{j k} \xi^{j} \eta^{k}, \quad j \geq 0, k \geq 0,
$$

with the $f_{j k}$ constant, about some arbitrary origin in that surface. A choice for $\xi$ and $\eta$ is

$$
\begin{aligned}
& \xi \equiv \psi^{1 / 2} \cos (\theta), \\
& \eta \equiv \psi^{1 / 2} \sin (\theta),
\end{aligned}
$$

so that $\psi^{1 / 2}$ corresponds to the "radial-like" coordinate and $\theta$ to the "polar-like" coordinate.

The square root of the toroidal flux, $\psi^{1 / 2}$, is chosen to correspond to the radial coordinate within Eqs. (4.24) and (4.25), instead of the flux itself, $\psi$. This selection was made since the toroidal flux is roughly $\psi \approx B_{0} \pi r^{2}$, with $r$ the minor radius from the magnetic axis to the flux surface. Thus, the function $f(\xi, \eta)$ can be expressed in the form

${ }^{74}$ Kuo-Petravic, G., and Boozer, A. H. (1987). Comp. Physics73, 107. 


$$
f(\psi, \theta)=\sum f_{j k} \psi^{(j+k) / 2}\{\cos (\theta)\}^{j}\{\sin (\theta)\}^{k}
$$

using Eqs. (4.23)-(4.25). The product $\{\cos (\theta)\}^{j}\{\sin (\theta)\}^{k}$ of Eq. (4.26) can be Fourier expanded as

$$
\{\cos (\theta)\}^{j}\{\sin (\theta)\}^{k}=\sum\left\{a_{m} \sin (m \theta)+b_{m} \cos (m \theta)\right\}
$$

with the range of $m$ given by $0 \leq m \leq j+k$, with $m$ even (odd) if $j+k$ is even (odd). Therefore, $f(\psi, \theta)$ can be expressed in the form

$$
f(\psi, \theta)=\sum_{m=0}^{\infty} \psi^{m / 2}\left\{a_{m}(\psi) \sin (m \theta)+b_{m}(\psi) \cos (m \theta)\right\}
$$

with $a_{m}(\psi)$ and $b_{m}(\psi)$ analytic functions of $\psi$, i.e.,

$$
a_{m}(\psi)=a_{m, 0}+a_{m, 2} \psi+a_{m, 4} \psi^{2}+\cdots,
$$

with $a_{m, 0}, a_{m, 2}, a_{m, 4}$, etc., constants.

Suppose that a third coordinate, $\varphi$, is then defined such that the reciprocal of the spatial Jacobian, $\{\nabla \psi \times \nabla \theta\} \cdot \nabla \varphi$, is finite and non-zero everywhere within the region of interest. We will also assume that the coordinate $\varphi$ is periodic, so that $\{\psi, \theta, \varphi\}$ form a toroidal coordinate system. Then Eqs. (4.28) and (4.29) imply that 
any function which is analytic within a given toroidal region can be cast in the form of Eq. (4.21).

IV-D. Expansion about the Magnetic Axis

The three functions $\{X(\psi, \theta, \varphi), Y(\psi, \theta, \varphi), Z(\psi, \theta, \varphi)\}$ within the spatial position of Eq. (4.18) are analytic in the absence of islands or stochastic regions. Hence, these functions can all be expressed in the analytic form of Eqs. (4.21) and (4.22), or more specifically,

$$
X(\psi, \theta, \varphi)=\varepsilon X_{1}(\theta, \varphi)+\varepsilon^{2} X_{2}(\theta, \varphi)+\varepsilon^{3} X_{3}(\theta, \varphi)+\cdots,
$$

with

$$
\begin{aligned}
& x_{1}(\theta, \varphi)= x_{1,1 s}(\varphi) \sin (\theta)+x_{1,1 c}(\varphi) \cos (\theta), \\
& x_{2}(\theta, \varphi)=x_{2,0}(\varphi)+x_{2,2 s}(\varphi) \sin (2 \theta)+x_{2,2 c}(\varphi) \cos (2 \theta), \\
& x_{3}(\theta, \varphi)=x_{3,1 s}(\varphi) \sin (\theta)+x_{3,1 c}(\varphi) \cos (\theta) \\
&+x_{3,3 s}(\varphi) \sin (3 \theta)+x_{3,3 c}(\varphi) \cos (3 \theta), \\
& x_{4}(\theta, \varphi)=x_{4,0}(\varphi)+x_{4,2 s}(\varphi) \sin (2 \theta)+x_{4,2 c}(\varphi) \cos (2 \theta) \\
&+x_{4,4 s}(\varphi) \sin (4 \theta)+x_{4,4 c}(\varphi) \cos (4 \theta),
\end{aligned}
$$

etc. These expressions for $\{X(\psi, \theta, \varphi), Y(\psi, \theta, \varphi), Z(\psi, \theta, \varphi)\}$ are simply power series expansions about a magnetic axis in terms of a dimensionless parameter, 


$$
\varepsilon \equiv\left\{\psi\left(k_{\max }\right)^{2} / B_{\min }\right\}^{1 / 2},
$$

with $1 / K_{\max }$ the scale length and $B_{\min }$ the scale magnetic field strength. Obviously, the expansion parameter, $\varepsilon$, is proportional to the square root of the toroidal magnetic flux, $\psi^{1 / 2}$.

The only scale length that appears in vacuum magnetic fields is the radius of curvature of the magnetic axis. We choose $K_{\max }$ to be the maximum local curvature of the axis and $B_{\min }$ to be the minimum magnetic field strength of the axis, so that the expansion parameter, $\varepsilon$, is maximized. This maximization of $\varepsilon$ insures that the expansion coefficients are all of order unity or less. The expansion parameter is approximately $\varepsilon \approx r / R_{c}$, with $r$ the minor radius from the axis to the outermost flux surface, and $R_{c}$ the minimum radius of curvature of the axis. Since $R_{c}$ does not necessarily correspond with the global toroidal radius of curvature of the axis, the expansion parameter, $\varepsilon$, is not necessarily the traditional inverse aspect ratio.

The reciprocal of the square of the magnetic field strength, $1 / B^{2}(\psi, \theta, \varphi)$, and the function $\beta_{*}(\psi, \theta, \varphi)$ are each expanded in a form similar to that of $\{X(\psi, \theta, \varphi), Y(\psi, \theta, \varphi), Z(\psi, \theta, \varphi)\}$, with the addition of a zeroth order term that is a function of only $\varphi$, i.e.,

$$
1 / B^{2}(\psi, \theta, \varphi)=\left\{1 /\left\{B_{0}(\varphi)\right\}^{2}\right\}+\varepsilon\left[1 / B^{2}\right]_{1}(\theta, \varphi)+\varepsilon^{2}\left[1 / B^{2}\right]_{2}(\theta, \varphi)+\cdots
$$




$$
\beta_{*}(\psi, \theta, \varphi)=\beta_{*_{0}}(\varphi)+\varepsilon \beta_{*_{1}}(\theta, \varphi)+\varepsilon^{2} \beta_{*_{2}}(\theta, \varphi)+\cdots .
$$

The poloidal expansions of $\left[1 / B^{2}\right]_{1}(\theta, \varphi), \beta_{*_{2}}(\theta, \varphi)$, etc., have the form of Eqs. (4.31)-(4.34). The rotational transform, $l(\psi)$, the poloidal electric current, $G(\psi)$, the toroidal electric current, $I(\psi)$, and the plasma pressure, $p(\psi)$, are simply Taylor expanded in terms of $\psi$,

$$
L(\psi)=l_{0}+\varepsilon^{2} l_{2}+\varepsilon^{4} l_{4}+\cdots
$$

with $\imath_{0}, \imath_{2}, \imath_{4}$, etc., constants.

In the remainder of this dissertation, the explicit dimensionality of all of the functions has been removed. That is, the factors of $\kappa_{\max }, B_{\min }$ and $\mu_{0}$ have been removed from all of the equations in this analysis. Hence, the expansion coefficients, $X_{1}(\theta, \varphi), X_{2}(\theta, \varphi), p_{2}, \beta_{*_{0}}(\varphi)$, etc., are all dimensionless.

\section{IV-E. Vacuum Magnetic Fields with Perfect Surfaces}

Nonzero plasma pressure and electric current provide only a slight modification of the method used to study vacuum magnetic fields. Therefore, we will first perform a detailed investigation 75 of vacuum magnetic fields with perfectly torodial flux surfaces. We will then outline the adjustments necessary for analyzing forcefree magnetic fields and scalar-pressure plasma equilibria in the two following sections.

${ }^{75}$ Garren, D. A., and Boozer, A. H. (1991). Phys. Fluids B: Plasma Physics 3, 2805. 
For vacuum magnetic fields, the plasma force balance equation, $j \times B=\nabla p$ of Eq. (1.1), is trivially satisfied, and the covariant form of the magnetic field of Eq. (2.12) reduces to

$$
B_{\text {cov }}=G_{0} \nabla \varphi \text {. }
$$

For vacuum fields, the toroidal coordinate, $\varphi$, becomes the magnetic potential of Laplace's equation, $\nabla^{2} \Phi=0$. The non-zero constant $G_{0}$ of Eq. (4.39) is the poloidal current in the field coils that generate the toroidal magnetic field (see Fig. 2). The equality of the two forms of the magnetic field, Eq. (4.15), then gives the vector differential equation

$$
G_{0}(\partial x / \partial \psi) \times(\partial x / \partial \theta)=(\partial x / \partial \varphi)+L(\psi)(\partial x / \partial \theta)
$$

Using the Frenet formulae of Eqs. (3.1)-(3.4) and the chain rule of calculus, the three components of this matching constraint are

$$
\begin{aligned}
\hat{k}_{0}: \quad G_{0}\{(\partial Y / \partial \psi)(\partial Z / \partial \psi)- & (\partial Z / \partial \psi)(\partial Y / \partial \theta)\} \\
= & \Xi(\psi, \theta, \varphi)+L(\psi)(\partial X / \partial \theta), \\
\hat{\tau}_{0}: \quad G_{0}\{(\partial Z / \partial \psi)(\partial X / \partial \theta)- & (\partial X / \partial \psi)(\partial Z / \partial \theta)\} \\
& =Y(\psi, \theta, \varphi)+l(\psi)(\partial Y / \partial \theta),
\end{aligned}
$$


$\hat{b_{0}}: \quad G_{0}\{(\partial X / \partial \psi)(\partial Y / \partial \theta)-(\partial Y / \partial \psi)(\partial X / \partial \theta)\}$

$$
=\Lambda(\psi, \theta, \varphi)+L(\psi)(\partial z / \partial \theta) \text {, }
$$

with

$\Xi(\psi, \theta, \varphi) \equiv(\partial X / \partial \varphi)+(d \ell / d \varphi)\{\tau[\ell(\varphi)] Y(\psi, \theta, \varphi)+k[\ell(\varphi)] Z(\psi, \theta, \varphi)\}$,

$$
\gamma(\psi, \theta, \varphi) \equiv(\partial Y / \partial \varphi)-(d \ell / d \varphi) \tau[\ell(\varphi)] X(\psi, \theta, \varphi),
$$

$$
\Lambda(\psi, \theta, \varphi) \equiv(\partial Z / \partial \varphi)+(d \ell / d \varphi)\{1-\kappa[\ell(\varphi)] X(\psi, \theta, \varphi)\}
$$

Similarly, the expression for the magnetic field strength is given by

$$
\begin{aligned}
& 1 / B^{2}(\psi, \theta, \varphi)=\left\{(d \ell / d \varphi)^{2}\right. \\
& +2(d \ell / d \varphi)\{(\partial Z / \partial \varphi)+2(\psi)(\partial Z / \partial \theta) \\
& -(d \ell / d \varphi) k(\ell(\varphi)) \times(\psi, \theta, \varphi)\} \\
& +\{(\partial X / \partial \varphi)+L(\psi)(\partial X / \partial \theta) \\
& +(d \ell / d \varphi)\{z[\ell(\varphi)] Y(\psi, \theta, \varphi)+k(\ell(\varphi)) Z(\psi, \theta, \varphi)\}\}^{2} \\
& +\{(\partial Y / \partial \varphi)+L(\psi)(\partial Y / \partial \theta) \\
& -(d \ell / d \varphi) \tau(\ell(\varphi)) \times(\psi, \theta, \varphi) r^{2} \\
& +\{(\partial Z / \partial \varphi)+2(\psi)(\partial Z / \partial \theta) \\
& \left.-(d \ell / d \varphi) k(\ell(\varphi)) \times(\psi, \theta, \varphi)\}^{2}\right\} /\left\{G_{0}\right\}^{2} \text {, }
\end{aligned}
$$

using Eq. (4.17)

In order that the spatial position, $x(y, \theta, \varphi)$ of Eq. (4.18), and 
the rotational transform, $L(\psi)$, give a vacuum field with perfect flux surfaces, the three components of the matching constraint of Eqs. (4.41)-(4.43) must hold through all orders in $\varepsilon$, or identically, $\psi^{1 / 2}$. We begin by solving these equations at $\varepsilon^{0}$ and $\varepsilon^{1}$ orders, and then elicit the constraints involved in successively higher orders. Application of this procedure will indicate a general methodolgy for constructing vacuum magnetic fields with perfect surfaces through all orders in $\varepsilon$.

IV-E-1. Vacuum Magnetic Fields through Zeroth Order Application of the three matching constraints of Eqs. (4.41)(4.43) at $\varepsilon^{0}$ order gives

$\hat{k}_{0}$ at $\varepsilon^{0}: \quad Y_{1}(\theta, \varphi)\left(\partial Z_{1} / \partial \theta\right)-Z_{1}(\theta, \varphi)\left(\partial Y_{1} / \partial \theta\right)=0$,

$\hat{\tau}_{0}$ at $\varepsilon^{0}: \quad Z_{1}(\theta, \varphi)\left(\partial X_{1} / \partial \theta\right)-X_{1}(\theta, \varphi)\left(\partial Z_{1} / \partial \theta\right)=0$,

$\hat{b_{0}}$ at $\varepsilon^{0}: \quad\left\{G_{0} / 2\right\}\left\{X_{1}(\theta, \varphi)\left(\partial Y_{1} / \partial \theta\right)-Y_{1}(\theta, \varphi)\left(\partial X_{1} / \partial \theta\right)\right\}=(d \ell / d \varphi)$.

The $\varepsilon^{0}$ order term of the magnetic field strength is

$$
1 /\left\{B_{0}(\varphi)\right\}^{2}=\left\{1 / G_{0}\right\}^{2}(d \ell / d \varphi)^{2},
$$

using Eq. (4.47). Integration of Eq. (4.51) through the entire length $L$ of the magnetic axis yields 


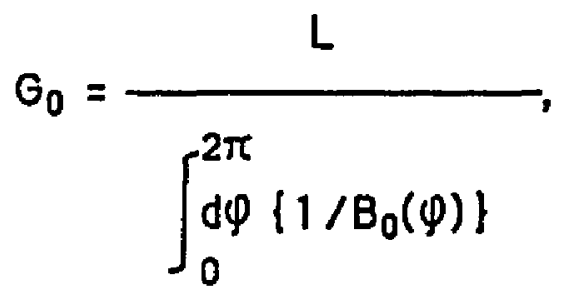

for the total poloidal electric current in the coils.

We can solve for the function $Z_{1}(\theta, \varphi)$, using Eqs. (4.48) and (4.49). The product of Eq. (4.48) and $X_{1}(\theta, \varphi)$ added to the product of Eq. (4.49) and $Y_{1}(\theta, \varphi)$ gives

$$
Z_{1}(\theta, \varphi)(d \ell / d \varphi)=0
$$

The function $z_{1}(\theta, \varphi)$ can be non-zero only for values of $\varphi$ in which $(d \ell / d \varphi)$ vanishes. Eq. (4.51) implies that the function $(d \ell / d \varphi)$ vanishes only for values of $\varphi$ in which the axis field strength, $B_{0}(\varphi)$, is infinite. Since singular magnetic field strengths are clearly not possible, the function $(d \ell / d \varphi)$ must be non-zero for all $\varphi$. The nonvanishing of the function $(d \ell / d \varphi)$ within Eq. (4.53) then implies that the function $Z_{1}(\theta, \varphi)$ is constrained to be zero for all values of $\theta$ and $\varphi$. Consequently, the vacuum $\hat{k}_{0}$ and $\hat{\tau}_{0}$ constraints at $\varepsilon^{0}$ order, Eqs. (4.48) and (4.49), hold trivially.

The only non-trivial vacuum constraint at $\varepsilon^{0}$ order, the $\hat{b}_{0}$ constraint of Eq. (4.50), becomes 


$$
\tilde{b}_{0} \text { at } \varepsilon^{0}: \quad\left\{G_{0} / 2\right\}\left\{X_{1,1 c}(\varphi) Y_{1,1 s}(\varphi)-Y_{1,1 c}(\varphi) X_{1,1 s}(\varphi)\right\}=(d \ell / d \varphi) \text {, }
$$

using the poloidal expansions for $X_{1}(\theta, \varphi)$ and $Y_{1}(\theta, \varphi)$ given in Eq. (4.31). The function $(d \ell / d \varphi)$ is a free function of $\varphi$, provided that it never vanishes. Therefore, Eq. (4.54) is one constraint involving the first order functions $X_{1,1 s}(\varphi), X_{1,1 c}(\varphi), Y_{1,1 s}(\varphi)$, and $Y_{1,1 c}(\varphi)$ of Eq. (4.31). Hence, this equation will be reserved for the first order set of constraints developed within the next subsection.

The previous analysis has shown that the zeroth order set of equations are trivial. This result follows since the magnetic axis alone characterizes toroidal plasma equilibria through zeroth order in the expansion. The analysis developed within Chapter III can be used to generate magnetic axes of vacuum magnetic fields with perfect flux surfaces.

\section{IV-E-2. Vacuum Magnetic Fields through First Order}

We now find the equations for vacuum equilibria through first order in the expansion parameter, $\varepsilon$. Application of the matching constraints of Eqs. (4.41)-(4.43) at $\varepsilon^{1}$ order produces

$$
\begin{aligned}
\hat{k}_{0} \text { at } \varepsilon^{1}: \quad\left\{G_{0} / 2\right\}\left\{Y_{1}(\theta, \varphi)\left(\partial Z_{2} / \partial \theta\right)-2 Z_{2}(\theta, \varphi)\left(\partial Y_{1} / \partial \theta\right)\right\} \\
=\left(\partial X_{1} / \partial \varphi\right)+l_{0}\left(\partial X_{1} / \partial \theta\right)+(d \ell / \partial \varphi) \tau(\varphi) Y_{1}(\theta, \varphi),
\end{aligned}
$$


$\hat{z}_{0}$ at $\varepsilon^{1}: \quad\left\{G_{0} / 2\right\}\left\{2 Z_{2}(\theta, \varphi)\left(\partial X_{1} / \partial \theta\right)-X_{1}(\theta, \varphi)\left(\partial Z_{2} / \partial \theta\right)\right\}$

$$
=\left(\partial Y_{1} / \partial \varphi\right)+l_{0}\left(\partial Y_{1} / \partial \theta\right)-(d \ell / \partial \varphi) \tau(\varphi) X_{1}(\theta, \varphi) \text {. }
$$

$\hat{b}_{0}$ at $\varepsilon^{1}: \quad\left\{G_{0} / 2\right\}\left\{X_{1}(\theta, \varphi)\left(\partial Y_{2} / \partial \theta\right)-2 Y_{2}(\theta, \varphi)\left(\partial X_{1} / \partial \theta\right)\right.$

$\left.+2 X_{2}(\theta, \varphi)\left(\partial Y_{1} / \partial \theta\right)-Y_{1}(\theta, \varphi)\left(\partial X_{2} / \partial \theta\right)\right\}$

$$
=-(d \ell / d \varphi) k(\varphi) X_{1}(\theta, \varphi)
$$

We substitute the poloidal expansions of the functions $X_{2}(\theta, \varphi)$, $Y_{2}(\theta, \varphi)$, and $Z_{2}(\theta, \varphi)$ of Eq. (4.32) into the three components of Eqs. (4.55)-(4.57). The resulting coefficients of $\sin (\theta)$ and $\cos (\theta)$ are then set equal to zero, so that Eqs. (4.55)-(4.57) hold for all values of the poloidal angle, $\theta$. This procedure yields the six equations

$$
\begin{aligned}
\hat{K}_{0} \text { at } \varepsilon^{1}, \sin (\theta): & G_{0}\left\{Y_{1,1 c}(\varphi)\left\{Z_{2,0}(\varphi)-Z_{2,2 c}(\varphi)\right\}-Y_{1,1 s}(\varphi) Z_{2,2 s}(\varphi)\right\} \\
& =\left(d X_{1,1 s} / d \varphi\right)-l_{0} X_{1,1 c}(\varphi)+(d \ell / d \varphi) r(\varphi) Y_{1,1 s}(\varphi) .
\end{aligned}
$$

$\hat{K}_{0}$ at $\varepsilon^{1}, \cos (\theta): G_{0}\left\{-Y_{1,1 s}(\varphi)\left\{Z_{2,0}(\varphi)+Z_{2,2 c}(\varphi)\right\}+Y_{1,1 c}(\varphi) Z_{2,2 s}(\varphi)\right\}$

$$
=\left(d X_{1,1 c} / d \varphi\right)+l_{0} X_{1,1 s}(\varphi)+(d \ell / d \varphi) \tau(\varphi) Y_{1,1 c}(\varphi) \text {. }
$$

$\hat{z}_{0}$ at $\varepsilon^{1}, \sin (\theta): \quad G_{0}\left\{-X_{1,1 c}(\varphi)\left\{Z_{2,0}(\varphi)-Z_{2,2 c}(\varphi)\right\}+X_{1,1 s}(\varphi) Z_{2,2 s}(\varphi)\right\}$

$$
=\left(d Y_{1,1 s} / d \varphi\right)-l_{0} Y_{1,1 c}(\varphi)-(d \ell / d \varphi) \tau(\varphi) X_{1,1 s}(\varphi) \text {, }
$$

$\hat{z}_{0}$ at $\varepsilon^{1}, \cos (\theta): \quad G_{0}\left\{X_{1,1 s}(\varphi)\left\{Z_{2,0}(\varphi)+Z_{2,2 c}(\varphi)\right\}-X_{1,1 c}(\varphi) Z_{2,2 s}(\varphi)\right\}$

$$
=\left(d Y_{1,1 c} / d \varphi\right)+l_{0} Y_{1,1 s}(\varphi)-(d \ell / d \varphi) z(\varphi) X_{1,1 c}(\varphi) \text {. }
$$




$$
\begin{aligned}
& \text { b. at } \varepsilon^{\dagger}, \sin (\theta): \quad G_{0}\left\{X_{1,1 c}(\varphi)\left\{Y_{2,0}(\varphi)-Y_{2,2 c}(\varphi)\right\}-X_{1,1 s}(\varphi) Y_{2,2 s}(\varphi)\right. \\
& \left.-Y_{1,1 c}(\varphi)\left\{X_{2,0}(\varphi)-X_{2,2 c}(\varphi)\right\}+Y_{1,1 s}(\varphi) X_{2,2 s}(\varphi)\right\} \\
& =-(d \ell / d \varphi) k(\varphi) X_{1,1 s}(\varphi) \text {. } \\
& \hat{b}_{0} \text { at } \varepsilon^{1}, \cos (\theta): \quad G_{0}\left\{-X_{1.1 s}(\varphi)\left\{Y_{2,0}(\varphi)+Y_{2,2 c}(\varphi)\right\}+X_{1,1 c}(\varphi) Y_{2,2 s}(\varphi)\right. \\
& \left.+Y_{1,1 s}(\varphi)\left\{X_{2,0}(\varphi)+X_{2,2 c}(\varphi)\right\}-Y_{1,1 c}(\varphi) Z_{2,2 s}(\varphi)\right\} \\
& =-(d \ell / d \varphi) k(\varphi) X_{1,1 c}(\varphi) \text {. }
\end{aligned}
$$

There is obviously sufficient freedom in the six functions of $\varphi$ within $X_{2}(\theta, \varphi)$ and $Y_{2}(\theta, \varphi)$, i.e., $X_{2,0}(\varphi), X_{2,2 s}(\varphi)$, etc., to satisfy the two vacuum $\hat{b}_{0}$ constraints at $\varepsilon^{1}$ order, Eqs. (4.62) and (4.63). However, there is not sufficient freedom in the three functions of $\varphi$ within $Z_{2}(\theta, \varphi)$ to solve the four $\hat{\kappa}_{0}$ and $\hat{\tau}_{0}$ constraints at $\varepsilon^{\mathfrak{l}}$ order, Eqs. (4.58)-(4.61). The magnetic axis curvature and torsion functions, $k(\varphi)$ and $\tau(\varphi)$, respectively, cannot generally be used to satisfy these constraints, since our expansion technique is valid for any smooth magnetic axis. Therefore, we must utilize the freedom in $X_{1}(\theta, \varphi)$ and $Y_{1}(\theta, \varphi)$, in addition to that within $Z_{2}(\theta, \varphi)$, to satisfy the $\hat{x}_{0}$ and $\hat{\tau}_{0}$ constraints at $\varepsilon^{1}$ order, Eq. (4.55) and (4.56). However, the functions $X_{1}(\theta, \varphi)$ and $Y_{1}(\theta, \varphi)$ must also satisfy the $\hat{b}_{0}$ constraint at $\varepsilon^{0}$ order, Eq. (4.50), as was shown in Sec IV-E-1. Summarizing, the functions $X_{1}(\theta, \varphi), Y_{1}(\theta, \varphi)$, and $Z_{2}(\theta, \varphi)$ must be used to satisfy the $\hat{b}_{0}$ constraint at $\varepsilon^{0}$ order, Eq. (4.54), and the $\hat{k}_{0}$ and $\hat{\varepsilon}_{0}$ constraints at $E^{1}$ order, Eqs. (4.58)-(4.61). We will refer to these constraints as the first order set of vacuum field constraints. 
The equations of the $\hat{\kappa}_{j}$ and $\hat{\varepsilon}_{0}$ constraints at $\varepsilon^{1}$ order, Eqs. (4.58)-(4.61), can be expressed in the form

$$
\begin{aligned}
& l_{0} V_{1}(\varphi)=X_{1,1 c}(\varphi)\left(d X_{1,1 s} / d \varphi\right)-X_{1,1 s}(\varphi)\left(d X_{1,1 c} / d \varphi\right) \\
&+ Y_{1,1 c}(\varphi)\left(d Y_{1,1 s} / d \varphi\right)-Y_{1,1 s}(\varphi)\left(d Y_{1,1 c} / d \varphi\right) \\
&+\left\{4 / G_{0}\right\}(d \ell / d \varphi)^{2} \tau(\varphi), \\
& Z_{2,0}(\varphi)=-\{1 /\{\theta(d \ell / d \varphi)\}\}\left(d V_{1} / d \varphi\right), \\
& Z_{2,2 s}(\varphi)=-\{1 /\{8(d \ell / d \varphi)\}\}\left\{\left(d V_{2} / d \varphi\right)-2 l_{0} V_{3}(\varphi)\right\}, \\
& Z_{2,2 c}(\varphi)=-\{1 /\{8(d \ell / d \varphi)\}\}\left\{\left(d V_{3} / d \varphi\right)+2 l_{0} V_{2}(\varphi)\right\},
\end{aligned}
$$

with

$$
\begin{gathered}
V_{1}(\varphi) \equiv\left\{X_{1,1 s}(\varphi)\right\}^{2}+\left\{X_{1,1 c}(\varphi)\right\}^{2}+\left\{Y_{1,1 s}(\varphi)\right\}^{2}+\left\{Y_{1,1 c}(\varphi)\right\}^{2} \\
V_{2}(\varphi) \equiv 2\left\{X_{1,1 s}(\varphi) X_{1,1 c}(\varphi)+Y_{1,1 s}(\varphi) Y_{1,1 c}(\varphi)\right\} \\
V_{3}(\varphi) \equiv\left\{X_{1,1 c}(\varphi)\right\}^{2}-\left\{X_{1,1 s}(\varphi)\right\}^{2}+\left\{Y_{1,1 c}(\varphi)\right\}^{2}-\left\{Y_{1,1 s}(\varphi)\right\}^{2}
\end{gathered}
$$

The second order configuration function $Z_{2}(\theta, \varphi)$ is completely determined by the first order functions $X_{1}(\theta, \varphi)$ and $Y_{1}(\theta, \varphi)$ via Eqs. (4.65)-(4.67). Hence, if the configuration is desired through only first order in $\varepsilon$, then Eqs. (4.65)-(4.67) can be safely ignored. Therefore, the only equations relevant to the configuration through 
$\varepsilon^{1}$ order are the $\hat{b}_{0}$ matching constraint at $\varepsilon^{0}$ order, Eq. (4.54), and the non-linear, first-order differential equation of Eq. (4.64).

The magnetic field strength through $\varepsilon^{1}$ order is given by

$$
B(\psi, \theta, \varphi)=B_{0}(\varphi)\left\{1+\varepsilon K(\varphi) X_{1}(\theta, \varphi)\right\}+\cdots,
$$

using Eq. (4.47). Thus, if the first order set of constraints holds, then all of the functions appearing in $1 / \mathrm{B}^{2}$ through first order are specified, so that the magnetic field strength is uniquely determined through first order. The result is not immediately obvious if some other form of the magnetic field strength than that of Eqs. (4.17) and $(4.47)$ is used.

The expression for the magnetic field strength in Eq. (4.71) gives the constraint that the first order variation of the field strength must not completely vanish, provided that the field strength itself never vanishes. This result implies that the magnetic field strength can be made uniform within a flux surface for toroidal plasma equilibria only in limits $^{76}$ in which the axis field strength vanishes or the flux surfaces become open. Such "isodynamic" or "omnigenous" equilibria were first proposed by Palumbo 77 .

We will now actually construct ${ }^{78}$ sample vacuum magnetic fields with perfect flux surfaces through first order in the ${ }^{76}$ Bernardin, M. P., Moses, R. W., and Tetaronis, J. A. (1986). Phys. Fluids 29, 2605.

77Palumbo, D. (1968). II Nuovo Cimento X53B, 507.

${ }^{78}$ Garren, D. A., and Boozer, A. H. (1991). Phys. Fluids B: Plasma Physics 3, 2805. 
expansion. The magnetic axis is presumed to be given by the results of Sec. III-B. We wish to retain the maximal freedom in the specification of the shape of the magnetic flux surfaces or the form of the magnetic field strength.

IV-E-2-a. Specification of Magnetic Flux Surface Shape

In order to determine the shape of the first order magnetic flux surfaces, it is convenient to express $X_{1}(\theta, \varphi)$ and $Y_{1}(\theta, \varphi)$ in the form 79

$$
\begin{aligned}
& X_{1}(\theta, \varphi)=\Gamma(\varphi)\{\cos (\theta-\gamma(\varphi))+\xi(\varphi) \cos (\theta-\gamma(\varphi)-\Delta(\varphi))\}, \\
& Y_{1}(\theta, \varphi)=\Gamma(\varphi)\{\sin (\theta-\gamma(\varphi))-\xi(\varphi) \sin (\theta-\gamma(\varphi)-\Delta(\varphi))\} .
\end{aligned}
$$

The shape of the constant $\psi$ surfaces to lowest order can be ascertained by the evaluation of $\{r(\theta, \varphi)\}^{2} \equiv\left\{X_{1}(\theta, \varphi)\right\}^{2}+\left\{Y_{1}(\theta, \varphi)\right\}^{2}$, giving

$$
\{r(\theta, \varphi)\}^{2}=\{\Gamma(\varphi)\}^{2}\left\{1+[\xi(\varphi)]^{2}+2 \xi(\varphi) \cos (2 \theta-2 \gamma(\varphi)-\Delta(\varphi))\right\}
$$

The function $r(\theta, \varphi)$ is proportional the distance from the magnetic axis to a particular point on a given magnetic flux surface. The form of $r(\theta, \varphi)$ in Eq. (4.74) indicates that the flux surfaces are ellipses through lowest order. The function $\Gamma(\varphi)\left\{1+\{\xi(\varphi)\}^{2}\right\}^{1 / 2}$ is the ${ }^{79}$ Garren, D. A., and Boozer, A. H. (1991). Phys. Fluids B: Plasma Physics 3, 2805. 
average minor radius of the ellipse. The function $\xi(\varphi)$ is a measure of the ellipticity, and the function $\Delta(\varphi)$ is a measure of the phase of rotation of the ellipse. The periodic function $\gamma(\varphi)$ is set by the constraint that the rotational transform on axis be constant, as will be shown later in this subsection.

Examination of Eq. (4.74) reveals that $\xi(\varphi)=0$ gives perfectly circular flux surfaces through first order, and $\xi(\varphi) \rightarrow 1$ yields flux surfaces that pinch off to infinitely thin ribbons. Application of the axis field strength of $\mathrm{Eq} .(4.51)$ and the $\hat{\mathrm{b}}_{0}$ constraint at $\varepsilon^{0}$ order of Eq. (4.54) produces

$$
\{\Gamma(\varphi)\}^{2}=2 /\left\{B_{0}(\varphi)\left\{1-[\xi(\varphi)]^{2}\right\}\right\}
$$

Hence, the corresponding average minor radius, $\Gamma(\varphi)\left\{1+[\xi(\varphi)]^{2}\right\}^{1 / 2}$, becomes singular in this limit, assuming non-zero field strength. That is, the flux surfaces become ribbons that are both infinitely thin and infinitely tall as the ellipticity, $\xi(\varphi)$, approaches unity.

The first-order, non-linear differential equation of Eq. (4.64) yields the following relation for the rotational transform on $\operatorname{axis}^{80}$,

$$
l_{0}=d \gamma / d \varphi+v(\varphi)
$$

with

${ }^{80}$ Garren, D. A., and Boozer, A. H. (1991). Phys. Fluids B: Plasma Physics 3, 2805. 


$$
v(\varphi) \equiv\left\{\left\{1-\{\xi(\varphi)\}^{2}\right\}(d \ell / d \varphi) \tau(\varphi)+\{\xi(\varphi)\}^{2}(d \Delta / d \varphi)\right\} /\left\{1+\{\xi(\varphi)\}^{2}\right\}
$$

Therefore, the rotational transform on axis is given by

$$
l_{0}=\{1 /(2 \pi)\} \int_{0}^{2 \pi} d \varphi v(\varphi),
$$

so that the periodic function $\gamma(\varphi)$ of Eq. $(4.76)$ serves to insure that $l_{0}$ is a constant. The rotational transform near the axis for vacuum fields is produced by 81 the torsion of the magnetic axis and the ellipticity, as is evident from Eqs. (4.76) and (4.77). The ellipticity tends to reduce the contribution due to the the torsion, but it tends to increase the contribution due to the change in the orientation of the ellipse as $\varphi$ varies, i.e., the contribution due to $d \Delta / d \varphi=0$.

IV-E-2-b. Specification of Magnetic Field Stength

Since particle drift trjectories are determined 82 by only the magnetic field strength in terms of Boozer coordinates, a more valuable procedure is to find equilibria corresponding to desirable forms of the field strength. The general form of the magnetic field strength in Boozer coordinates is

$$
B(\psi, \theta, \varphi)=B_{0}(\varphi)\{1+\varepsilon \delta(\varphi) \cos (\theta-\alpha(\varphi))\},
$$

${ }^{81}$ Spitzer Jr., L. (1958). Phys. Fluids 1, 253.

82Boozer, A. H. (1984). Phys. Fluids 27; 2441. 
through first order in $\varepsilon$. We wish to retain the maximal freedom in the $\varphi$-functions, $B_{0}(\varphi), \delta(\varphi)$, and $\alpha(\varphi)$. The field strength of Eq. (4.79) can be expressed in the form 83

$$
B(\psi, \theta, \varphi)=B_{0}(\varphi)\left\{1+\varepsilon\left(2 / B_{0}(\varphi)\right\}^{1 / 2} K(\varphi) \eta(\varphi) \cos (\theta-\alpha(\varphi))\right\},
$$

with the lowest order flux surfaces given by

$$
\begin{gathered}
X_{1}(\theta, \varphi)=\left\{2 / B_{0}(\varphi)\right\}^{1 / 2} \eta(\varphi) \cos (\theta-\alpha(\varphi)), \\
Y_{1}(\theta, \varphi)=\left\{2 / B_{0}(\varphi)\right\}^{1 / 2}\{1 / \eta(\varphi)\}\{\sin (\theta-\alpha(\varphi))+\sigma(\varphi) \cos (\theta-\alpha(\varphi))\} .
\end{gathered}
$$

These forms for $X_{1}(\theta, \varphi)$ and $Y_{1}(\theta, \varphi)$ are completely general, but only three of the four $\varphi$-functions $B_{0}(\varphi), \eta(\varphi), \sigma(\varphi)$, and $\alpha(\varphi)$ are unconstrained. It is convenient to choose the $\varphi$-functions $B_{0}(\varphi)$, $\eta(\varphi)$, and $\alpha(\varphi)$ to be arbitrary, so that the magnetic field strength through first order is freely specified. The $\varphi$-function $\sigma(\varphi)$ is then constrained to satisfy the first-order, non-linear differential equation

$$
\begin{aligned}
d \sigma / d \varphi=\left\{2 G_{0} / B_{0}(\varphi)\right\}\{\eta(\varphi)\}^{2} \tau(\varphi) \\
-\left\{2_{0}-(d \alpha / d \varphi)\right\}\left\{1+\{\eta(\varphi)\}^{4}+\{\sigma(\varphi)\}^{2}\right\}
\end{aligned}
$$

with the periodicity condition $\sigma(\varphi=0)=\sigma(\varphi=2 \pi)$. Solutions to Eq. ${ }^{83}$ Garren, D. A., and Boozer, A. H. (1991). Phys. Fluids B: Plasma Physics 3, 2805. 
(8.43) are found by integrating it forward in $\varphi$, minimizing the . quantity $\sigma(\varphi=0)-\sigma(\varphi=2 \pi)$ by varying either the initial point of integration, $\sigma(\varphi=0)$, or the value of the axis transform, $L_{0}$. The remainder of the variables that enter are free, except for the constraints that $K[\ell(\varphi)]$ and $\tau[\ell(\varphi)]$ are consistent with a closed curve and that $B_{0}(\varphi)$ and $\eta(\varphi)$ never vanish or become infinite. The solution obtained for $\sigma(\varphi)$ need not be unique, since Eq. (4.83) is non-linear.

The previous functions of $\varphi$ that conveniently described the magnetic flux surfaces can be represented in terms of those that characterize the magnetic field strength via the relations 84

$$
\begin{gathered}
\{\Gamma(\varphi)\}^{2}=\frac{\left\{\left\{\{\eta(\varphi)\}^{2}+1\right\}^{2}+\{\sigma(\varphi)\}^{2}\right\}}{2 B_{0}(\varphi)\{\eta(\varphi)\}^{2}}, \\
\{\xi(\varphi)\}^{2}=\frac{\left\{\{\eta(\varphi)\}^{2}-1\right\}^{2}+\{\sigma(\varphi)\}^{2}}{\left\{\{\eta(\varphi)\}^{2}+1\right\}^{2}+\{\sigma(\varphi)\}^{2}}, \\
\gamma(\varphi)=\alpha(\varphi)-\tan ^{-1}\left[\frac{\sigma(\varphi)}{\{\eta(\varphi)\}^{2}+1}\right], \\
\Delta(\varphi)=\tan ^{-1}\left[\frac{\sigma(\varphi)}{\{\eta(\varphi)\}^{2}-1}\right]+\tan ^{-1}\left[\frac{\sigma(\varphi)}{\{\eta(\varphi)\}^{2}+1}\right] .
\end{gathered}
$$

Approximately circular flux surfaces are obtained if $\eta(\varphi)$ is near ${ }^{84}$ Garren, D. A., and Boozer, A. H. (1991). Phys. Fluids B: Plasma Physics 3, 2805. 
unity and $\sigma(\varphi)$ is near zero. The flux surfaces pinch off to ribbons that are both infinitely thin and infintely tall as $|\eta(\varphi)|$ approaches zero or infinity or as $|\sigma(\varphi)|$ approaches infinity.

The magnetic field strength of a general vacuum configuration with perfect surfaces has the form of Eq. (4.79) with

$$
\delta(\varphi)=\left\{2 / B_{0}(\varphi)\right\}^{1 / 2} k(\varphi) \eta(\varphi)
$$

using Eq. (4.80), which implies that there exists a large degree of freedom in the shape of the flux surfaces. Configurations with widely varying magnetic axes can have the same form of the magnetic field strength through first order. For example, the flux surfaces of the five-period helical configuration shown in Fig. 12 and the three-period knotted configuration of Fig. 13 both possess a field strength of the form of Eq. (4.79) with $B_{0}(\varphi)=1, \delta(\varphi)=1.25$, and $\alpha(\varphi)=N \varphi$. The magnetic axes of Figs. 12 and 13 are given by Figs. 5 and 7 , respectively. The flux surfaces of Figs. 12 and 13 are examples of configurations which are quasi-helically symmetric through first order, since their field strengths through first order are functions of only one helical angle, $\alpha \equiv \theta-N \varphi$, within the flux surfaces. We examine quasi-helical symmetry in greater detail within Chapter VI.

IV-E-3. Vacuum Magnetic Fields through Higher Order The vacuum constraint equations at second and higher orders 


\section{FGURE 12}

\section{FIVE PERIOD HELICAL CONFIGURATION}

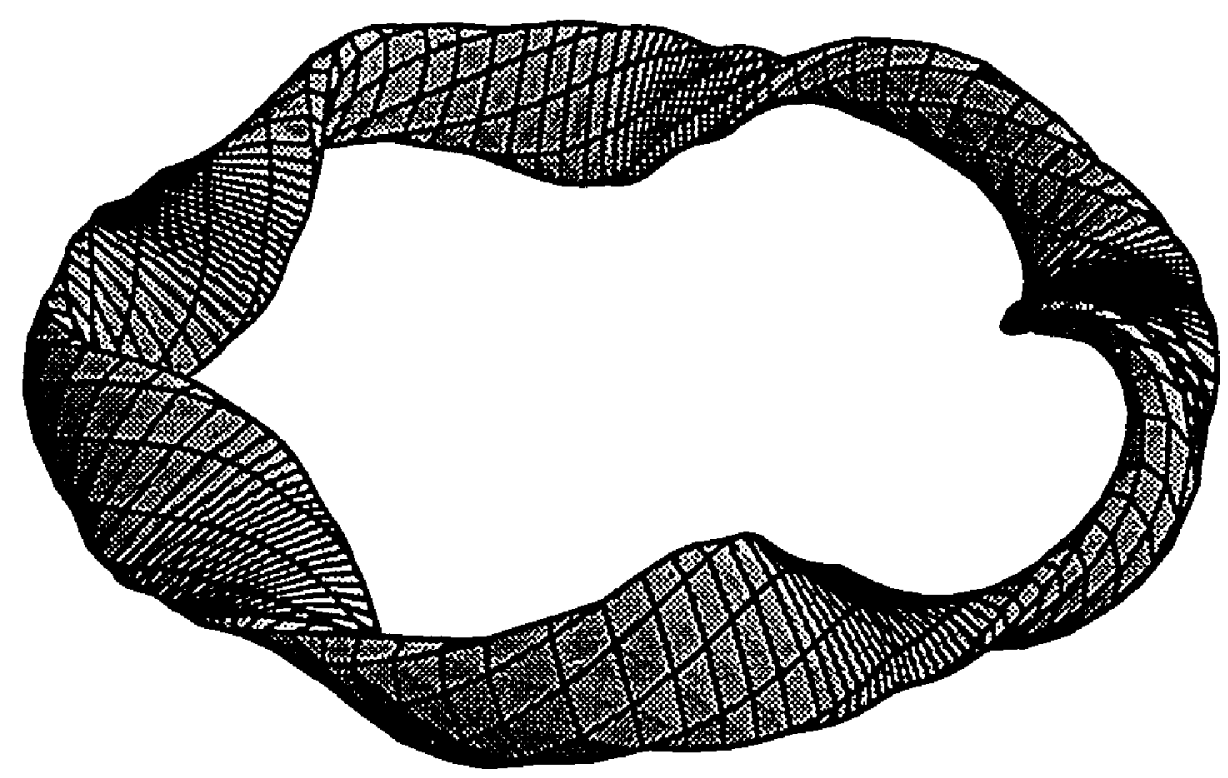

A flux or $\psi$ surface of a conventional five-period helical configuration with a "quasi-helically symmetric" magnetic field strength through first order given by Eq. (4.79), with $B_{0}(\varphi)=1$, $\delta(\varphi)=1.25$, and $\alpha(\varphi)=N \varphi$. The magnetic axis of this configuration corresponds to that of Figs. 1 and 6 . The toroidal lines show the true constant $\theta$ surfaces through lowest order for Figs. 12, 13, 14, 16 , and 18, but the poloidal lines were chosen by the adaptive step size Runge-Kutta routine used, and hence do not represent true constant $\varphi$ surfaces. 
FIGURE 13

\section{THREE PERIOD KNOTTED CONFIGURATION}

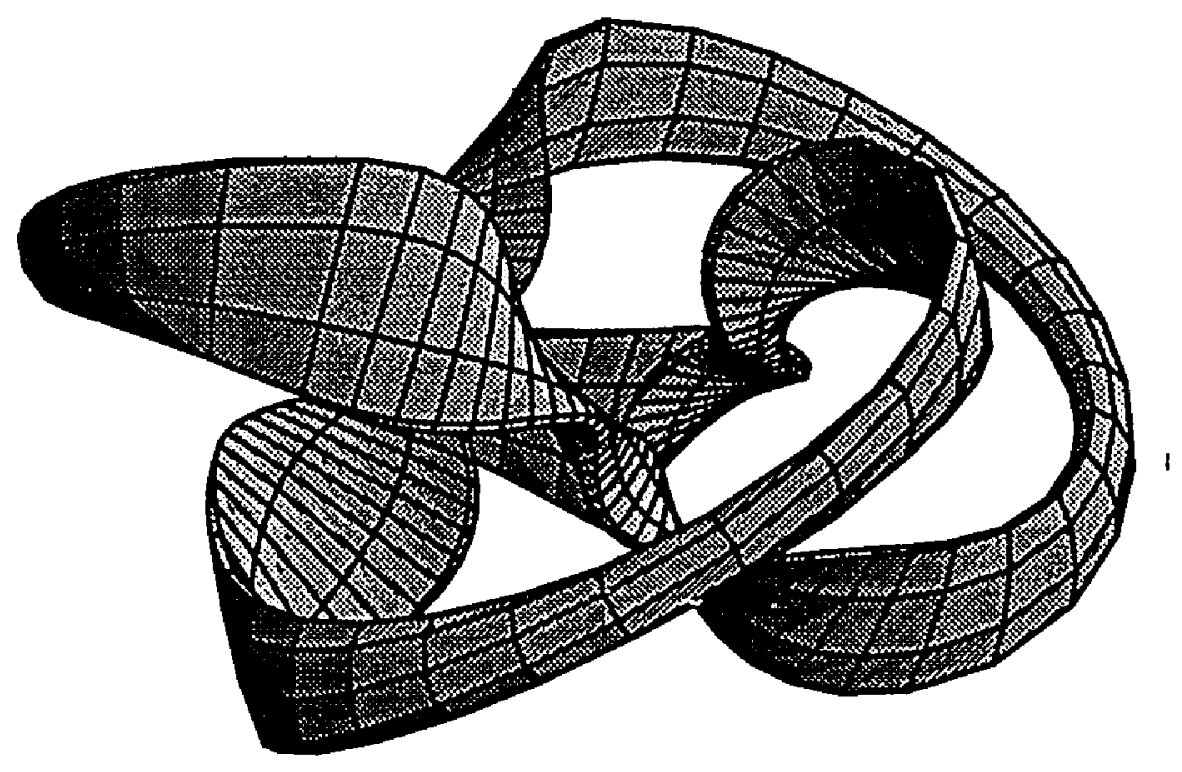

A flux surface of a three-period knotted configuration with the same magnetic field strength in Boozer coordinates through first order as that corresponding to the five-period helical configuration of Fig. 12. The magnetic axis of this configuration corresponds that of Figs. 7 and 8. 
follows the construction of the first order set of constraints. We will present the second order set of vacuum constraints and then give the generic scheme 85 for eliciting the higher order constraint equations.

In Sec. IV-E-2, we found that the two $\hat{b}_{0}$ constraints at $\varepsilon^{1}$ order, Eqs. (4.62) and (4.63), could be satisfied by the six functions $\varphi$ within $X_{2}(\theta, \varphi)$ and $Y_{2}(\theta, \varphi)$. These constraints were not associated with the first order set of constraints, since they did not require lower order functions of $\varphi$ to be free, as did the $\hat{k}_{0}$ and $\hat{z}_{0}$ constraints at $\varepsilon^{1}$ order, (4.58)-(4.61).

We now examine the three components of matching the two forms of the magnetic field, Eqs. $(4.41)-(4.43)$, at $\varepsilon^{2}$ order. The poloidal expansions of the functions $X_{3}(\theta, \varphi), Y_{3}(\theta, \varphi)$, and $Z_{3}(\theta, \varphi)$ shown in Eq. (4.33) imply that each component yields three independent constraints, i.e., one each for matching the $\varphi$-dependent coefficients of the $\sin (2 \theta), \cos (2 \theta)$, and constant terms. The highest order functions that enter both the $\hat{\kappa}_{0}$ and $\hat{z}_{0}$ constraints of $\varepsilon^{2}$ order are $X_{2}(\theta, \varphi), Y_{2}(\theta, \varphi)$, and $Z_{3}(\theta, \varphi)$. The highest order functions that enter into the $\hat{b}_{0}$ constraint are $X_{3}(\theta, \varphi), Y_{3}(\theta, \varphi)$, and $Z_{2}(\theta, \varphi)$. The eight functions of $\varphi$ within the poloidal expansions of $X_{3}(\theta, \varphi)$ and $Y_{3}(\theta, \varphi)$ are sufficient to satisfy the three $\varphi$-constraints of the $\hat{b}_{0}$ matching constraint at $\varepsilon^{2}$ order. However, the four functions of $\varphi$ within $Z_{3}(\theta, \varphi)$, i.e., $Z_{3,1 s}(\varphi), Z_{3,1 c}(\varphi), Z_{3,3 s}(\varphi)$, and $Z_{3.3_{C}}(\varphi)$ of Eq. $(4.43)$ are not sufficient to satisfy the six ${ }^{85}$ Garren, D. A., and Boozer, A. H. (1991). Phys. Fluids B: Plasma Physics 3, 2805. 
independent $\varphi$-constraints of the $\hat{k}_{0}$ and $\hat{\varepsilon}_{0}$ constraints at $\varepsilon^{2}$ order.

Following the pattern set by first order, the delimma of satisfying these second order equations is ameliorated by combining the $\hat{\kappa}_{0}$ and $\hat{\varepsilon}_{0}$ constraint equations at $\varepsilon^{2}$ order and the $\hat{b}_{0}$ constraint equations at $\varepsilon^{1}$ order into the second order set of vacuum constraint equations. The functions of $\varphi$ within $X_{2}(\theta, \varphi), Y_{2}(\theta, \varphi)$, and $Z_{3}(\theta, \varphi)$ are sufficient to satisfy this set of constraint equations.

We can represent the six independent $\varphi$-constraints of the $\hat{\mathbf{K}}_{0}$ and $\hat{\tau}_{0}$ constraints at $\varepsilon^{2}$ order in a less elusive fashion. This processs follows in an analogous fashion to that of obtaining the simplified forms of Eqs. (4.64)-(4.67) from the four independent $\varphi$ constraints of the $\hat{k}_{0}$ and $\hat{\tau}_{0}$ constraints at $\varepsilon^{1}$ order, Eqs. (4.58)(4.61). Four of the six resulting equations determine the function $Z_{3}(\theta, \varphi)$, i.e., via the $\varphi$-functions $Z_{3,1 s}(\varphi), z_{3,1 c}(\varphi), Z_{3,3 s}(\varphi)$, and $Z_{3,3 c}(\varphi)$. The remaining equations are two non-linear, first-order differential equations in terms of second and lower order configuration quantities, i. $\theta ., X_{2}(\theta, \varphi), Y_{2}(\theta, \varphi)$, etc.

We will now give a general methodology for satisfying the vacuum constraint equations, Eqs. (4.41)-(4.43), through arbitrary order in the expansion parameter, $\varepsilon$. The functions $X_{j}(\theta, \varphi), Y_{j}(\theta, \varphi)$, and $Z_{j+1}(\theta, \varphi)$ must be used to satisfy the $\hat{b}_{0}$ matching constraint at $\varepsilon^{j-1}$ order and the $\hat{\kappa}_{0}$ and $\hat{\tau}_{0}$ matching constraints at $\varepsilon^{j}$ order. More specifically, we use the $j+1$ free functions of $\varphi$ in each of the functions $X_{j}(\theta, \varphi)$ and $Y_{j}(\theta, \varphi)$, and the $j+2$ free functions of $\varphi$ within $Z_{j+1}(\theta, \varphi)$ to satisfy the $j$ independent $\varphi$-constraints in the $\vec{b}_{0}$ 
matching constraint at $\varepsilon^{j-1}$ order and the $j+1$ independent $\varphi$ constraints in each of the $\hat{k}_{0}$ and $\hat{z}_{0}$ matching constraints at $\varepsilon^{j}$ order. We denote these particular constraints as the jth set of matching constraints. That is, the $3 j+2$ independent equations of the $j$ th order set of matching constraints must be satisfied by the $3 j+4$ functions of $\varphi$ that first enter into these equations. Obviously, each new set of constraints introduces two new free functions of $\varphi$ that may be used to give some freedom in specifying the magnetic configuration. As will be shown in Chapter $V$, the freedom of two functions of $\varphi$ for each order in the expansion is sufficient to permit nearly arbitrary freedom in the magnetic field strength on one particular flux surface.

This methodology for satisfying the constraint equations of Eqs. (4.41)-(4.43) follows from the vanishing of the function $Z_{1}(\theta, \varphi)$ for general toroidal magnetic fields with perfect flux surfaces. The peculiar procedure for satisfying the constraint equations implies that the functions $X(\psi, \theta, \varphi)$ and $Y(\psi, \theta, \varphi)$, given through some order $\varepsilon^{j}$, constrain the form of $Z(\psi, \theta, \varphi)$ through the next higher order, $\varepsilon^{j+1}$. That is, the function $Z(\psi, \theta, \varphi)$, which gives the dominant contribution to the shape of the magnetic potential or $\varphi$ surfaces, is subdominant to the functions $X(\Psi, \theta, \varphi)$ and $Y(\psi, \theta, \varphi)$, which give the dominant contribution to the shape of the flux or $\psi$ surfaces. This result is merely a direct consequence of the divergence-free property of general magnetic fields and requiring nested flux surfaces. 
We now examine the complications involved in constructing vacuum magnetic fields with perfect surfaces through some order. Assume that we wish to find a magnetic configuration through some order, say $\varepsilon^{j}$, in the spatial position, $x(\psi, \theta, \varphi)$. We apply the results of first and second order to infer a generic scheme for finding $X(\psi, \theta, \varphi)$ through arbitrary order. Sec. IV-E-2 revealed that one first-order, non-linear differential equation, in addition to the more trivial $\hat{b}_{0}$ constraint at $\varepsilon^{0}$ order, must be satisfied to give the first order flux surfaces. For flux surfaces through second order, two first-order, non-linear differential equations and the more trivial $\hat{b_{0}}$ constraints at $\varepsilon^{1}$ order must hold. Extending this procedure through arbitrary order implies that $j$ first-order differential equations must hold in specifying the configuration through $\varepsilon^{j}$ order, via $x(\psi, \theta, \varphi)$, assuming that the configuration is given through one lower order, $\varepsilon^{j-1}$. This result follows from the fact that the $j+2$ functions of $\varphi$ within $Z_{j+1}(\theta, \varphi)$ can be used to satisfy all but jof the $2\{j+1\} \hat{k}_{0}$ and $\hat{\tau}_{0} \varphi$-constraints at $\varepsilon^{j}$ order.

\section{IV-F. Force-Free Magnetic Fields}

We now extend the results of the previous section regarding magnetic fields with perfectly nested toroidal surfaces to apply for magnetic configurations with force-free electric currents. The plasma force balance equation of Eq. (1.1) becomes

$$
j \times B=0,
$$


for such configurations, since no forces are exerted. Hence, no plasma pressure gradient can exist within force-free magnetic fields. Eq. (4.89) obviously implies that the magnetic field and the electric current must lie in the same direction, so that the current can be expressed in the form

$$
j(x)=\alpha(x) B(x),
$$

with $\alpha(x)$ some scalar function of position. Taking the divergence of Eq. (4.90) then yields

$$
B \cdot \nabla \alpha=0,
$$

So that the magnetic field lines and electric current trajectories lie within surfaces of constant $\alpha(x)$. Thus, function $\alpha(x)$ gives nested toroidal magnetic surfaces, provided that $\nabla \alpha$ is well-defined everywhere except on the magnetic axis.

The covariant representation of the magnetic field of Eq. (2.13) reduces to

$$
B_{c o v}=G(\psi) \nabla \varphi+I(\psi) \nabla \theta,
$$

for force-free configurations. This form follows since the function $\beta_{*}(\psi, \theta, \varphi)$ of Eq. (2.13) is proportional ${ }^{86}$ to the plasma pressure gradient, as demonstrated within Sec. IV-A. The partial differential ${ }^{86}$ Boozer, A. H. (1981). Phys Fluids 24, 1999. 
equations resulting from the equality of the two forms of the magnetic field, Eq. (4.15), becomes ${ }^{87}$

$$
\begin{aligned}
(\partial x / \partial \varphi)+l(\psi)(\partial x / \partial \theta)=G(\psi) & (\partial x / \partial \psi) \times(\partial x / \partial \theta) \\
& +I(\psi)(\partial x / \partial \varphi) \times(\partial x / \partial \psi)
\end{aligned}
$$

The three components of this matching constraint are given by

$$
\begin{aligned}
& \hat{k}_{0}: \quad G(\psi)\{(\partial Y / \partial \psi)(\partial Z / \partial \psi)-(\partial Z / \partial \psi)(\partial Y / \partial \theta)\} \\
&+I(\psi)\{(\partial Z / \partial \psi) \Upsilon(\psi, \theta, \varphi)-(\partial Y / \partial \psi) \Lambda(\psi, \theta, \varphi)\}=\Xi(\psi, \theta, \varphi)+l(\psi)(\partial X / \partial \theta), \\
& \hat{\tau}_{0}: \quad G(\psi)\{(\partial Z / \partial \psi)(\partial X / \partial \theta)-(\partial X / \partial \psi)(\partial Z / \partial \theta)\} \\
&+I(\psi)\{(\partial X / \partial \psi) \Lambda(\psi, \theta, \varphi)-(\partial Z / \partial \psi) \Xi(\psi, \theta, \varphi)\} \\
&= \Upsilon(\psi, \theta, \varphi)+l(\psi)(\partial Y / \partial \theta),
\end{aligned}
$$

$\hat{b}_{0}: \quad G(\psi)\{(\partial X / \partial \psi)(\partial Y / \partial \theta)-(\partial Y / \partial \psi)(\partial X / \partial \theta)\}$

$+I(\psi)\{(\partial Y / \partial \psi) \Xi(\psi, \theta, \varphi)-(\partial X / \partial \psi) \Upsilon(\psi, \theta, \varphi)\}$

$$
=\Lambda(\psi, \theta, \varphi)+l(\psi)(\partial Z / \partial \theta),
$$

with $\Xi(\psi, \theta, \varphi), \gamma(\psi, \theta, \varphi)$, and $\Lambda(\psi, \theta, \varphi)$ defined in Eqs. (4.44)-(4.46). The reciprocal of the field strength, $1 / B^{2}$, for force-free magnetic fields can be obtained by using Eq. (4.17), which gives 88

${ }^{87}$ Garren, D. A., and Boozer, A. H. (1991). Phys. Fluids B: Plasma Physics 3, 2805. ${ }^{88}$ Ibid. 


$$
\begin{aligned}
& 1 / B^{2}(\psi, \theta, \varphi)=\left\{(d \ell / d \varphi)^{2}\right. \\
& +2(d \ell / d \varphi)\{(\partial Z / \partial \varphi)+l(\psi)(\partial Z / \partial \theta) \\
& -(d \ell / d \varphi)<(\ell(\varphi)) \times(\psi, \theta, \varphi)\} \\
& +\{(\partial X / \partial \varphi)+2(\psi)(\partial X / \partial \theta) \\
& +(d \ell / d \varphi)\{\tau[\ell(\varphi)] Y(\psi, \theta, \varphi)+k(\ell(\varphi)) Z(\psi, \theta, \varphi)\}\}^{2} \\
& +\{(\partial Y / \partial \varphi)+2(\psi)(\partial Y / \partial \theta) \\
& -(d \ell / d \varphi) r(\ell(\varphi)) \times(\psi, \theta, \varphi)\}^{2} \\
& +\{(\partial Z / \partial \varphi)+l(\psi)(\partial Z / \partial \theta) \\
& \left.-(d \ell / d \varphi) K(\ell(\varphi)) \times(\psi, \theta, \varphi)\}^{2}\right\} /\{G(\psi)+L(\psi) I(\psi)\}^{2} .
\end{aligned}
$$

This equation can be otained by making the replacement

$$
\left(G_{0}\right)^{2} \rightarrow\{G(\psi)+\imath(\psi) l(\psi)\}^{2}
$$

within the form of $1 / B^{2}$ for the vacuum case, Eq. (4.47).

For force-free magnetic fields, the average force balance equation of Eq. (4.6) is no longer trivially satisfied. This equation becomes $^{89}$

$$
d G / d \psi+2(\psi)(d] / d \psi)=0
$$

using the fact that $\mathrm{dp} / \mathrm{d} \psi=0$ for force-free magnetic fields. Since a pressure gradient can not exist for force-free fields, the only admissible pressure profile is one of the form $p(\psi)=p_{0}$, with $p_{0}$ a ${ }^{89}$ Garren, D. A., and Boozer, A. H. (1991). Phys. Fluids B: Plasma Physics 3, 2805. 
constant.

The functions $G(\psi)$ and $I(\psi)$ of Eq. (4.99) can be Taylor expanded in the form of $2(\psi)$ given in Eq. (4.38). Finiteness of the toroidal electric current density on axis implies that the constant term within $I(\psi)$, i.e., $I_{0}$, must be zero. The average force balance equation of Eq. (4.99) indicates that one of the three flux functions $d G / d \psi, d l / d \psi$, and $L(\psi)$ is constrained by the other two, assuming all three functions are non-zero for all $\psi$. These three flux functions are further restricted if any of them have zeroes within the toroidal region.

IV-F-1. Force-Free Magnetic Fields through Zeroth Order

Application of the force-free constraint equations of Eqs. (4.94)-(4.96) at $\varepsilon^{0}$ order reveals that these equations are identical to those for vacuum fields. The only additional constraint is

$$
G_{2}=-l_{0} I_{2}
$$

with the constant $G_{2}$ the lowest order, poloidal electric current within the plasma. Eq. (4.100) is merely the average force balance equation of Eq. (4.99) at zeroth order in $\varepsilon$. Hence, the shape and field strength of the magnetic axis are unaffected by force-free effects at lowest order. Therefore, all of the results within Sec. IV-E-1 regarding magnetic axes of vacuum fields also hold for force-free magnetic fields. 
IV-F-2 Force-Free Magnetic Fields through First Order Modification of the vacuum field equations due to the effects of a non-zero, force-free electric current first occurs at $\varepsilon^{1}$ order. The first order set of matching constraints for force-free equilibria is given by

$$
\hat{b_{0}} \text { at } \varepsilon^{0}: \quad\left\{G_{0} / 2\right\}\left\{X_{1}(\theta, \varphi)\left(\partial Y_{1} / \partial \theta\right)-Y_{1}(\theta, \varphi)\left(\partial X_{1} / \partial \theta\right)\right\}=(d \ell / \partial \varphi) \text {, }
$$

$\vec{K}_{0}$ at $\varepsilon^{1}: \quad\left\{G_{0} / 2\right\}\left\{Y_{1}(\theta, \varphi)\left(\partial Z_{2} / \partial \theta\right)-2 Z_{2}(\theta, \varphi)\left(\partial Y_{1} / \partial \theta\right)\right\}$

$$
=\left(\partial X_{1} / \partial \varphi\right)+l_{0}\left(\partial X_{1} / \partial \theta\right)+(d \ell / \partial \varphi)\left[\tau(\varphi)+(1 / 2) l_{2}\right\} Y_{1}(\theta, \varphi) \text {, }
$$

$$
\begin{aligned}
\hat{\tau}_{0} \text { at } \varepsilon^{1}: \quad & \left\{G_{0} / 2\right\}\left\{2 Z_{2}(\theta, \varphi)\left(\partial X_{1} / \partial \theta\right)-X_{1}(\theta, \varphi)\left(\partial Z_{2} / \partial \theta\right)\right\} \\
= & \left(\partial Y_{1} / \partial \varphi\right)+l_{0}\left(\partial Y_{1} / \partial \theta\right)-(\partial \ell / \partial \varphi)\left\{\tau(\varphi)+(1 / 2) I_{2}\right\} X_{1}(\theta, \varphi) .
\end{aligned}
$$

These equations reveal that the replacement ${ }^{90}$

$$
\tau(\varphi) \rightarrow\left\{\tau(\varphi)+(1 / 2) I_{2}\right\}
$$

within the first order vacuum constraints of Eqs. (4.50), (4.55), (4.56) gives the first order set of force-free constraint equations. The resulting equations for $z_{2,0}(\varphi), z_{2,2 s}(\varphi)$, and $z_{2,2 c}(\varphi)$ are the same as that of the vacuum case, Eqs. (4.65)-(4.67), so that the function $Z_{2}(\theta, \varphi)$ is unaffected by force-free effects. However, the force-free form of Eq. (4.64) becomes

90Garren, D. A., and Boozer, A. H. (1991). Phys. Fluids B: Plasma Physics 3, 2805. 


$$
\begin{aligned}
l_{0} V_{1}(\varphi)=X_{1.1 c}(\varphi) & \left(d X_{1,1 s} / d \varphi\right)-X_{1.1 s}(\varphi)\left(d X_{1.1 c} / d \varphi\right) \\
+Y_{1.1 c}(\varphi)\left(d Y_{1.1 s} / d \varphi\right)-Y_{1.1 s}(\varphi)\left(d Y_{1.1 c} / d \varphi\right) & +\left\{4 / G_{0}\right\}(d \ell / d \varphi)^{2}\left\{\tau(\varphi)+(1 / 2) I_{2}\right\}
\end{aligned}
$$

The magnetic field strength for force-free magnetic fields through first order is given by Eq. (4.71), just as for the vacuum case. Hence, force-free electric currents do not modify the magnetic field strength through first order.

IV-F-2-a. Specification of Magnetic Flux Surfaces

The rotational transform on axis, $l_{0}$, for force-free magnetic fields contains one additional contribution, which is that due to the toroidal electric current density on axis, $I_{2}=(d l / d \psi)_{0}$. The actual equation for the rotational transform on axis can be obtained by applying the replacement $\tau(\varphi) \rightarrow\left\{\tau(\varphi)+(1 / 2) I_{2}\right\}$ within Eqs. (4.76) and (4.77), giving

$$
l_{0}=d \gamma / d \varphi+v(\varphi)
$$

with

$$
\begin{aligned}
v(\varphi) \equiv\{[1 & \left.\left.-\{\xi(\varphi)\}^{2}\right](d \ell / d \varphi)\left[\tau(\varphi)+(1 / 2) I_{2}\right]+\{\xi(\varphi)\}^{2}(d \Delta / d \varphi)\right\} \\
& \times\left\{1 /\left\{1+\{\xi(\varphi)\}^{2}\right\}\right\} .
\end{aligned}
$$

Together with the contributions due to torsion of the axis and the ellipticity of the flux surfaces, these effects give the means to 
produce 91 a rotational transform near the magnetic axis. This result will also be shown to apply for general, scalar-pressure equilibria within Sec. IV-G.

IV-F-2-b. Specification of Magnetic Field Strength

In constructing force-free magnetic fields with a desired form of the magnetic field strength through first order, the replacement $\tau(\varphi) \rightarrow\left\{\tau(\varphi)+(1 / 2) I_{2}\right\}$ should be applied to Eq. (4.83), yielding

$$
\begin{aligned}
d \sigma / d \varphi=\left\{2 G_{0} / B_{0}(\varphi)\right\} & \{(\varphi)\}^{2}\left\{\tau(\varphi)+(1 / 2) I_{2}\right\} \\
& -\left\{l_{0}-(d \alpha / d \varphi)\right\}\left\{1+\{\eta(\varphi)\}^{4}+\{\sigma(\varphi)\}^{2}\right\},
\end{aligned}
$$

The actual procedure for constructing force-free magnetic fields is given within Sec. IV-E-2-b, implementing Eq. (4.108), instead of Eq. (4.83).

IV-F-3 Force-Free Magnetic Fields through Higher Order

Close inspection of the constraint equations of Eqs. (4.94)(4.96) for force-free magnetic fields reveals that the leading order behavior of $X(\psi, \theta, \varphi), Y(\psi, \theta, \varphi)$, and $Z(\psi, \theta, \varphi)$ is unmodified ${ }^{92}$ from its behavior for the vacuum case. Terms resulting from force-free currents, i.e., those due to $G_{2}, G_{4}, \ldots$, and $I_{2}, I_{4}, \ldots$, never give the highest order contribution within the constraint equations of Eqs. (4.94)-(4.96) at any order. Hence, plasma current effects are

91 Spitzer Jr., L. (1958). Phys. Fluids 1, 253.

${ }^{92}$ Garren, D. A., and Boozer, A. H. (1991). Phys. Fluids B: Plasma Physics 3, 2805. 
subdominant to the vacuum field in the construction of the magnetic configuration. Therefore, the methodology used in the matching constraints for vacuum fields is not significantly modified by the inclusion of force-free currents.

The previous analysis demonstrates that the techniques for finding force-free magnetic fields is similar to that used in constructing vacuum magnetic fields with perfect surfaces. The average force balance equation of Eq. (4.99), which is a constraint on the flux functions $d G / d \psi, d I / d \psi$, and $\imath(\psi)$, is the only additional restriction on the system.

\section{IV-G. Scalar-Pressure Plasma Equilibria}

We now wish to determine whether this methodology for constructing force-free magnetic fields also applies for magnetic configurations with a non-zero pressure gradient. That is, we wish to extend the formalism to general, scalar-pressure, MHD equilibria.

The three components of the constraint resulting from the equality of the two forms of the magnetic field, Eq. (4.15), are given by 93

$$
\begin{aligned}
\hat{K}_{0}: \quad G(\psi) & \{(\partial Y / \partial \psi)(\partial Z / \partial \psi)-(\partial Z / \partial \psi)(\partial Y / \partial \theta)\} \\
+ & I(\psi)\{(\partial Z / \partial \psi) \Upsilon(\psi, \theta, \varphi)-(\partial Y / \partial \psi) \Lambda(\psi, \theta, \varphi)\} \\
& +\beta_{*}(\psi, \theta, \varphi)\{(\partial Y / \partial \theta) \Lambda(\psi, \theta, \varphi)-(\partial Z / \partial \theta) \Upsilon(\psi, \theta, \varphi)\} \\
& =\Xi(\psi, \theta, \varphi)+L(\psi)(\partial X / \partial \theta),
\end{aligned}
$$

${ }^{93}$ Garren, D. A., and Boozer, A. H. (1991). Phys. Fluids B: Plasma Physics 3, 2805. 
$\hat{\tau}_{0}: \quad G(\psi)\{(\partial z / \partial \psi)(\partial X / \partial \theta)-(\partial X / \partial \psi)(\partial z / \partial \theta)\}$

$+I(\psi)\{(\partial X / \partial \psi) \Lambda(\psi, \theta, \varphi)-(\partial Z / \partial \psi) \equiv(\psi, \theta, \varphi)\}$

$+\beta_{*}(\psi, \theta, \varphi)\{(\partial z / \partial \theta) \equiv(\psi, \theta, \varphi)-(\partial X / \partial \theta) \Lambda(\psi, \theta, \varphi)\}$

$=\gamma(\psi, \theta, \varphi)+2(\psi)(\partial Y / \partial \theta)$,

$\hat{b}_{0}: \quad G(\psi)\{(\partial X / \partial \psi)(\partial Y / \partial \theta)-(\partial Y / \partial \psi)(\partial X / \partial \theta)\}$

$+[(\psi)\{(\partial Y / \partial \psi) \equiv(\psi, \theta, \varphi)-(\partial X / \partial \psi) \Upsilon(\psi, \theta, \varphi)\}$

$+\beta_{*}(\psi, \theta, \varphi)\{(\partial X / \partial \theta) Y(\psi, \theta, \varphi)-(\partial Y / \partial \theta) \equiv(\psi, \theta, \varphi)\}$

$=\Lambda(\psi, \theta, \varphi)+i(\psi)(\partial z / \partial \theta)$.

with $\Xi(\psi, \theta, \varphi), \Upsilon(\psi, \theta, \varphi)$, and $\Lambda(\psi, \theta, \varphi)$ defined in Eqs. (4.44)-(4.46).

The expression for the reciprocal of the magnetic field strength, $1 / B^{2}$, is exactly the same as that for force-free magnetic fields, Eq. (4.97).

The only function that enters into the constraint equations of Eqs. (4.109)-(4.111) for toroidal, scalar-pressure equilibria that is absent within the force-free equations is $\beta_{*}(\psi, \theta, \varphi)$. This additional function is determined by the Fourier coefficients of the magnetic field strength via Eq. (4.7). The function $\beta_{*}(\psi, \theta, \varphi)$ will be shown to neither augment, nor remove, any freedom in the functions of $\varphi$ that exist within the force-free analysis.

IV-G-1. Scalar-Pressure Equilibria through Zeroth Order The $\varepsilon^{0}$ order equations are unmodified by the presence of a 
pressure gradient, just as for the force-free current case. Therefore, the magnetic axis analysis for vacuum fields given within Sec. IV-E-1 also applies to toroidal, scalar-pressure equilibria. The only modification is that the average force balance equation, Eq. (4.6), at lowest order, has the form

$$
G_{2}+l_{0} I_{2}+P_{2} G_{0} /\left\langle\left\{B_{0}(\varphi)\right\}^{2}\right\rangle=0
$$

for scalar-pressure equilibria. This equation constrains the lowest order poloidal current density within the plasma, $G_{2}$, given the toroidal current density, $I_{2}$, and plasma pressure gradient, $P_{2}$, to lowest order.

IV-G-2. Scalar-Pressure Equilibria through First Order

Finite pressure gradient modifications of the constraint equations first occur at $\varepsilon^{1}$ order. The first order set of constraint equations for scalar-pressure equilibria is given by

$$
\begin{aligned}
\hat{b_{0}} \text { at } \varepsilon^{0}: & \left\{G_{0} / 2\right\}\left\{X_{1}(\theta, \varphi)\left(\partial Y_{1} / \partial \theta\right)-Y_{1}(\theta, \varphi)\left(\partial X_{1} / \partial \theta\right)\right\}=(\partial \ell / \partial \varphi), \\
\hat{K}_{0} \text { at } \varepsilon^{1}: & \left\{G_{0} / 2\right\}\left\{Y_{1}(\theta, \varphi)\left(\partial Z_{2} / \partial \theta\right)-2 Z_{2}(\theta, \varphi)\left(\partial Y_{1} / \partial \theta\right)\right\} \\
= & \left(\partial X_{1} / \partial \varphi\right)+L_{0}\left(\partial X_{1} / \partial \theta\right)+(\partial \ell / \partial \varphi)\left\{\tau(\varphi)+(1 / 2) I_{2}\right\} Y_{1}(\theta, \varphi) \\
& -(\partial \ell / \partial \varphi) \beta_{H_{0}}(\varphi)\left(\partial Y_{1} / \partial \theta\right),
\end{aligned}
$$




$$
\begin{aligned}
\hat{\tau}_{0} \text { at } \varepsilon^{1}: & \left\{G_{0} / 2\right\}\left\{2 Z_{2}(\theta, \varphi)\left(\partial X_{1} / \partial \theta\right)-X_{1}(\theta, \varphi)\left(\partial Z_{2} / \partial \theta\right)\right\} \\
= & \left(\partial Y_{1} / \partial \varphi\right)+l_{0}\left(\partial Y_{1} / \partial \theta\right)-(d \ell / \partial \varphi)\left\{\tau(\varphi)+(1 / 2) I_{2}\right\} X_{1}(\theta, \varphi) \\
& +(d \ell / \partial \varphi) \beta_{*_{0}}(\varphi)\left(\partial X_{1} / \partial \theta\right) .
\end{aligned}
$$

Examination of these matching constraints reveals that the equation for the rotational transform on axis of Eq. (4.105) and the functions $Z_{2,2 s}(\varphi)$ and $Z_{2,2 c}(\varphi)$ of Eqs. (4.66) and (4.67) are not affected by a non-zero plasma pressure gradient. The function $\beta_{*_{0}}(\varphi)$, which is proportional to the lowest order pressure gradient, only modfies the second order function $Z_{2,0}(\varphi)$ via

$$
Z_{2.0}(\varphi)=-\{1 /\{8(d \ell / d \varphi)\}\}\left(d V_{1} / d \varphi\right)+\left(1 / G_{0}\right)(d \ell / d \varphi) B_{\#_{0}}(\varphi)
$$

with $V_{1}(\varphi)$ defined in Eq. (4.68).

The expression for the magnetic field strength through $\varepsilon^{1}$ order of scalar-pressure equilibria is identical to that for vacuum fields, Eq. (4.71). Therefore, the first order variation in the magnetic field strength of general scalar-pressure equilibria is determined by the curvature of the magnetic axis, so that all other effects enter at higher order. This result is consistent with the $1 / R$ dependence of the magnetic field strength of a tokamak with toroidal radius $R$.

The previous analysis has demonstrated 94 that a non-zero plasma pressure gradient only modifies the second order function ${ }^{94}$ Garren, D. A., and Boozer, A. H. (1991). Phys. Fluids B: Plasma Physics 3, 2805. 
$Z_{2}(\theta, \varphi)$, and thus does not affect the magnetic field strength or the shape of the flux surfaces through first order. Hence, the force-free results regarding the specification of the magnetic flux surface shape and the magnetic field strength through first order, which were given within Sec. IV-F-2, also apply for scalar-pressure equilibria.

Suppose that the magnetic field strength on axis is Fourier decomposed in the form of Eq. (4.4),

$$
1 /\left\{B_{0}(\varphi)\right\}^{2}=\left\{1 /\left\langle\left\{B_{0}(\varphi)\right\}^{2}\right\rangle\right\}\left\{1+\sum_{n=1}^{\infty}\left\{\gamma_{n} \cos (n \varphi)+\lambda_{n} \sin (n \varphi)\right\}\right\},
$$

with $\gamma_{n}$ and $\lambda_{n}$ constants and $\left\langle\left\{B_{0}(\varphi)\right\}^{2}\right\rangle$ the average of $\left\{B_{0}(\varphi)\right\}^{2}$ within a flux surface, as defined by Eq. (4.5). The function $\beta_{*_{0}}(\varphi)$ then has the form

$$
\beta_{*_{0}}(\varphi)=\left\{p_{2} G_{0} /\left\langle\left\{B_{0}(\varphi)\right\}^{2}\right\rangle\right\} \sum_{n=1}^{\infty}\{1 / n\}\left\{\gamma_{n} \sin (n \varphi)-\lambda_{n} \cos (n \varphi)\right\},
$$

using Eq. (4.7). A given magnetic axis, lowest order plasma pressure gradient, $P_{2}$, and axis magnetic field strength, $B_{0}(\varphi)$, uniquely determine the function $B_{*_{0}}(\varphi)$ via Eq. (4.118). Since $\beta_{*_{0}}(\varphi)$ is completely determined by functions that enter at zeroth order, a non-zero plasma pressure gradient neither augments, nor 
removes95, any freedom within the first order constraint equations. We now extend these results to higher order.

\section{IV-E-3 Scalar-Pressure Equilibria through Higher Order}

Inspection of the matching constraints of Eqs. (4.109)-(4.111)

shows that the leading order behavior of $X(\psi, \theta, \varphi), Y(\psi, \theta, \varphi)$, and $Z(\psi, \theta, \varphi)$ is unchanged ${ }^{96}$ from that of the vacuum and force-free cases. Terms due to a non-zero pressure gradient and electric current, i.e., those involving any of the factors $p_{2}, p_{4}, \ldots, G_{2}, G_{4}, \ldots$, and $I_{2}, l_{4}, \ldots$, never give the highest order contribution to the matching constraints of Eqs. (4.109)-(4.111) at any order. The vacuum magnetic field, which is generated by electric currents outside of the plasma, always gives the leading order terms.

The only new function of position that appears within the scalar-pressure constraint equations of Eq. (4.109)-(4.111) is $\beta_{*}(\psi, \theta, \varphi)$. This function is completely determined by the form of the magnetic field strength and the flux functions, $G(\psi), l(\psi), l(\psi)$, and $p(\psi)$, via $\mathrm{Eq} .(4.7)$. Hence, the function $\beta_{*}(\psi, \theta, \varphi)$ does not introduce any true freedom into the system of equations. Furthermore, we will now demonstrate that a non-zero $\beta_{*}(\psi, \theta, \varphi)$ does not affect the basic methodology 97 of satisfying the matching constraints that was used for the vacuum and force-free field cases.

Sec. IV-E-3 revealed that the $j$ th order set of matching

95Garren, D. A., and Boozer, A. H. (1991). Phys. Fluids B: Plasma Physics 3, 2805. 96/bid.

97 Ibid. 
constraints is given by the $\hat{\boldsymbol{K}}_{0}$ and $\hat{\boldsymbol{\varepsilon}}_{0}$ matching constraints at $\varepsilon^{\mathrm{j}}$ order and the $\hat{b}_{0}$ matching constraint at $\varepsilon^{j-1}$ order. The highest order non-flux functions that appear in both the $\hat{\boldsymbol{k}}_{0}$ and $\hat{\boldsymbol{\tau}}_{0}$ matching constraints at $\varepsilon^{j}$ order are given by $X_{j}(\theta, \varphi), Y_{j}(\theta, \varphi), z_{j+1}(\theta, \varphi)$, and $\beta_{* j-1}(\theta, \varphi)$. For the $\hat{b}_{0}$ matching constraint at $\varepsilon^{j-1}$ order, the highest order functions are $X_{j}(\theta, \varphi), Y_{j}(\theta, \varphi), Z_{j-1}(\theta, \varphi)$, and $\beta_{* j}$

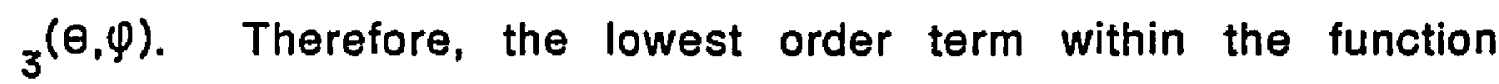
$\beta_{*}(\psi, \theta, \varphi)$ that enters into the $\mathrm{j}$ th order set of matching constraints is $\beta_{*_{j-1}}(\theta, \varphi)$. Thus, the function $\beta_{*_{j}}(\theta, \varphi)$ first enters into the $(j+1) \underline{\text { st }}$ order set of matching constraints.

We now have all the necessary equations for constructing toroidal, scalar-pressure MHD equilibria order by order in the expansion. As noted earlier, the function $\beta_{*_{j}}(\theta, \varphi)$ first enters into the $(j+1)$ st set of matching constraints. The form of $\beta_{*}(\psi, \theta, \varphi)$ in Eq. (4.7) implies that $\beta_{* j}(\theta, \varphi)$ is determined by the form of the reciprocal of the field strength, $1 / B^{2}$, through $\varepsilon^{j}$ order. The leading order function that enters $1 / B^{2}$ through $\varepsilon^{j}$ order is the $X_{j}(\theta, \varphi)$ factor within the term $-2 \varepsilon^{j}(d \ell / d \varphi)^{2} k(\varphi) X_{j}(\theta, \varphi)$ of Eq. (4.97). The function $X_{j}(\theta, \varphi)$ is associated with the $j$ th order set of matching constraints. All other variables that enter $1 / B^{2}$ through $\varepsilon^{j}$ order determined by lower order sets of matching constraints. Thus, the function $\beta_{* j}(\theta, \varphi)$ is also determined by the $j \frac{j \text { th }}{j}$ and lower order sets of matching constraints. However, $\beta_{* j}(\theta, \varphi)$ first enters the $(j+1)$ st 
set of matching constraints. Therefore, for each order that a term in the expansion of $\beta_{*}(\psi, \theta, \varphi)$ first enters into the constraint equations, it is completely determined by one lower order.

The only additional constraint that must hold is the average force balance equation, Eq (4.6). This relation involves the flux functions $d p / d \psi, G(\psi), l(\psi), L(\psi)$, and $\left\langle B^{2}\right\rangle$, but does not affect the basic methodology of satisfying the equality of the two forms of the magnetic field. Therefore, the techniques used to satisfy the constraints for vacuum and force-free magentic fields is not significantly modified by including a plasma pressure gradient within the toroidal region.

\section{IV-H. Physical Interpretation of Ordering}

The techniques outlined within this dissertation can be used to investigate the spectrum of possible three-dimensional plasma equilibria. Such equilibria are typically characterized by certain parameters, such as magnetic field strength, rotational transform, plasma current, and others. There exist sufficient approximations of these parameters near the magnetic axis of any equilibrium. We will qualify 98 the particular order in the expansion in which each of the relevant parameters first enters. We find that the parameters of primary interest to the fusion program have suitable approximations through third order in the expansion.

${ }^{98}$ Garren, D. A., and Boozer, A. H. (1991). Phys. Fluids B: Plasma Physics 3, 2805. 


\section{IV-H-1. Zeroth Order}

Zeroth order in the expansion is characterized entirely by the magnetic axis itself. The shape of this magnetic field line is arbitrary, provided that it is a smooth, closed curve. In addition, the magnetic field strength of the axis itself is arbitrary. However, this axis field strength must be non-zero in order to apply the methods within this dissertation.

\section{IV-H-2. First Order}

Through first order in the expansion, the cross sections of the magnetic flux surfaces are ellipses. The magnitude of the ellipticity, $\xi(\varphi)$, can vary with the toroidal angle, $\varphi$. In addition, the elliptical flux surfaces can also rotate with $\varphi$ and generate a contribution to the rotational transform on axis, $\iota_{0}$, as was demonstrated within Sec. IV-E-2.

The magnetic field strength at first order is governed by the magnitude of the curvature of the axis, regardless of the plasma current and pressure profiles, as was shown in Sec. IV-G-2. As the magnetic axis curves and twists through space, the magnetic field strength is stronger on the inboard side of the local curvature, and it is weaker on the outboard side.

The form of the rotational transform on axis, $l_{0}$, is apparent at this order. The toroidal electric current on axis, $I_{2}$, the torsion of the axis, $\tau_{0}(\varphi)$, and the ellipticity, $\xi(\varphi)$, can all contribute to the transform on axis, as was demonstrated in Sec. IV-G-2. 
Obviously, the magnetic flux surfaces can admit a non-zero plasma pressure gradient, $P_{2}$, and poloidal electric current, $G_{2}$, near the axis. The only constraint on these flux functions is that they satisfy the lowest order, average force balance equation of Eq. (4.112).

Another characteristic of toroidal configurations is the shear of the magnetic field lines. The field line shear is defined to be the change in the pitch 99 of neighboring magnetic field lines. Desirable plasma equilibria should have large local shear ${ }^{100}$ in regions of bad curvature in order to mitigate certain pressure-driven plasma instabilities. The interrelation of the freedom in the local shear coupled with that of the magnetic field strength would be a formidable, but useful, topic for future investigation. The lowest order magnetic flux surfaces give the dominant contribution to the local shear, as will be demonstrated within the following two paragraphs.

The local shear $s(\psi, \theta, \varphi)$ in terms of Boozer coordinates is defined by 101

$$
\begin{aligned}
S(\psi, \theta, \varphi) & \equiv|\nabla \psi|^{-4}\{\{B \times \nabla \psi\} \cdot\{\nabla \times\{B \times \nabla \psi\}\}\} \\
& =(1 / J)(d \imath / d \psi)+B \cdot \nabla\left\{\left\{G(\psi) g_{\psi \theta}-I(\psi) g_{\varphi \psi}\right\} /\left\{J|\nabla \psi|^{2}\right\}\right\},
\end{aligned}
$$

${ }^{99}$ Friedberg, J. P. (1987). Ideal Magneto-Hydrodynamics, Plenum Press, New York, p. 72.

${ }^{100}$ Nührenberg, J., and Zille, R. (1987). Theory of Fusion Plasmas, Proceedings of the workshop held at Villa Cipressi-Varenna, Italy, Aug. 24-28, Societa Italiana di Fisica, Bologna, Italy, p. 3.

101 ibid. 
with $J(\psi, \theta, \varphi)$ the spatial Jacobian of Eq. (4.14) and $g_{\psi \theta}$ and $g_{\varphi \psi}$ the magnetic field metrics defined by

$$
\begin{aligned}
& g_{\psi \theta} \equiv(\partial x / \partial \psi) \cdot(\partial x / \partial \theta), \\
& g_{\varphi \psi} \equiv(\partial x / \partial \varphi) \cdot(\partial x / \partial \psi) .
\end{aligned}
$$

Using the expansion functions defined within this disseration, the lowest order expression for the magnetic field line shear becomes

$$
\begin{aligned}
S(\psi, \theta, \varphi) & =\left\{G_{0} /\left\{4(d \ell / \partial \varphi)^{2} \varepsilon^{2}\right\}\right\} \\
& \times\left\{(\partial / \partial \varphi)+l_{0}(\partial / \partial \theta)\right\}\left[\frac{(\partial / \partial \theta)\left\{\left(X_{1}(\theta, \varphi)\right\}^{2}+\left\{Y_{1}(\theta, \varphi)\right\}^{2}\right\}}{\left(\partial X_{1} / \partial \theta\right)^{2}+\left(\partial Y_{1} / \partial \theta\right)^{2}}\right] .
\end{aligned}
$$

The lowest order expression for the shear obviously depends on the details of the configuration. The lowest order non-trivial flux surfaces, which are determined by the functions $X_{1}(\theta, \varphi)$ and $Y_{1}(\theta, \varphi)$, gives the dominant contribution to the local shear.

The lowest order contribution to the local shear of Eq. (4.119) diverges as the plasma radius approaches zero, as exhibited by the $1 / \varepsilon^{2}$ dependence of Eq. (4.122). This peculiar result is consistent with the fact that the global shear, i.e., the $(d L / d \psi)$ term of Eq. (4.119), is a $\varepsilon^{0}$ order term. The global shear is independent of the toroidal and poloidal angles, $\theta$ and $\varphi$, respectively, unlike the local shear. The global shear, which is given by $l_{2} / l_{0}$ within our analysis, 
enters the equilibrium equations at third order in $\varepsilon$, whereas the lowest order flux surfaces which determine the local shear enter at first order in $\varepsilon$. Thus, the local shear enters the equilibrium equations two orders lower than the global shear, which is consistent with the fact that the lowest order local shear of Eqs. (4.119) and (4.122) is two orders lower than the global shear.

\section{IV-H-3. Second Order}

The second order flux surfaces can exhibit triangular contributions to the flux surface cross section. Additionally, the center of the flux surface cross section can be shifted with respect to the magnetic axis. This offset of the flux surfaces with respect to the axis is called the Shafronov shift ${ }^{102}$. The actual flux surface shape can be evaluated using the function

$$
\{r(\psi, \theta, \varphi)\}^{2} \equiv\{X(\psi, \theta, \varphi)\}^{2}+\{Y(\psi, \theta, \varphi)\}^{2}
$$

with $X(\psi, \theta, \varphi)$ and $Y(\psi, \theta, \varphi)$ given through second order in $\varepsilon$. The function $r(\psi, \theta, \varphi)$ gives the distance from the magnetic axis to a point determined by a set of coordinates, $\{\psi, \theta, \varphi\}$. Flux surface cross sections are determined by varying over the angles $\theta$ and $\varphi$, while holding the toroidal flux, $\psi$, fixed.

The parameters that determine the shape of the flux surfaces through second order are interrelated with the average magnetic ${ }^{102}$ Shafranov, V. D. (1966). In Reviews of Plasma Physics, edited by M. A. Leontovich, Consultants Bureau, New York, Vol. II. 
well, which first enters the form of the field strength at second order. The average magnetic well is defined to be the derivative of the average magnetic field strength within a flux surface, with respect the flux coordinate, $\psi$, as described within the introduction. The average magnetic well, in addition to the field line shear, can serve to stabilize ${ }^{103}$ a plasma against certain pressure-driven instabilities.

Many other effects give contributions to the magnetic field strength at second order. The toroidal and poloidal plasma currents near the axis, $I_{2}$ and $G_{2}$, respectively, the plasma pressure gradient near the axis, $p_{2}$, the torsion of the axis, $\tau(l)$, the curvature of the axis, $k(l)$, the rotatioal transform on axis, $2_{0}$, and products of these terms all contribute to the magnetic field strength at second order. This result is obtained by examination of the general formula for the magnetic field strength of Eq. (4.97). Due to our inability to make any insightful simplifications, we did not explicitly give the field strength through second order within this dissertation.

\section{IV-H-4. Third Order}

At third order in the expansion, the flux surface shape can exhibit a contribution due to bean-shapedness, in addition to an enhancement of the ellipticity. The bean-shapedness arises from $M=4$ poloidal harmonics within the magnetic field strengh, just as triangularity arises from poloidal harmonics with $M=3$. The shape of ${ }^{103}$ Friedberg, J. P. (1987). Ideal Magneto-Hydrodynamics, Plenum Press, New York, p. 73. 
the flux surfaces is discerned via $\{r(\psi, \theta, \varphi)\}^{2}$ of Eq. (4.123), using $X(\psi, \theta, \varphi)$ and $Y(\psi, \theta, \varphi)$ evaluated through third order. The magnetic field strength of Eq. (4.97) through third order contains contributions due to the global shear, $l_{2} / l_{0}$, and higher order plasma current and pressure quantities, such as $G_{4}, l_{4}$, and $P_{4}$.

A toroidal plasma equilibrium can possess a net toroidal electric current that is driven by a non-zero pressure gradient ${ }^{104}$. Since the pressure gradient must vanish at the magnetic axis for analytic magnetic fields, this electric current must also vanish on axis. The lowest order value of this so-called "bootstrap current" is given by $I_{4}$ within our analysis.

The dependence of the bootstrap current on the geometric properties of the magnetic configuration can be extracted using the rotational transform, $L(\psi)$, and the Fourier harmonics within the magnetic field strength, $B(\psi, \theta, \varphi)$, as outlined by Boozer and Gardner ${ }^{105}$. The details of their results are beyond the scope of this dissertation and will thus not be given. However, the relevant point is that approximations permit the computation of a self-consistent value for the bootstrap current near the magnetic axis of any toroidal plasma equilibrium.

${ }^{104}$ Nishkawa, K., and Wakatani, M. (1990). Plasma Physics: Basic Theory with Fusion Applications, Springer-Verlag Berlin Heidelberg, Germany, p. 266.

105Boozer, A. H., and Gardner, H. (1991). Phys Fluids B: Plasma Physics 2, 2408. 


\section{CHAPTER V}

\section{RESTRICTIONS ON THE MAGNETIC FIELD STRENGTH}

We are able to elicit several generic restrictions ${ }^{106}$ on the form of the magnetic field strength corresponding to general toroidal plasma equilibria. For equilibria in which the magnetic field strength never vanishes within the toroidal region, (1) the first order curvature term in the magnetic field strength cannot be made to completely vanish, and (2) the magnetic field strength is not arbitrary through third order in the expansion parameter, $\varepsilon$. However, we also clarify the specific freedoms in the form of the magnetic field stength. These freedoms are that: (1) the magnetic field strength is arbitrary through second order in $\varepsilon$, provided that the first order curvature term does not entirely vanish, and (2) the magnetic field strength on one particular magnetic flux surface is fully arbitrary, again with the provision that the first order curvature term is non-zero.

\section{A. Non-Vanishing of the First Order Curvature Term}

The magnetic field strength through $\varepsilon^{1}$ order for toroidal, scalar-pressure equilibria is given by Eq. (4.71). The first order term in this expression is $\varepsilon k(\varphi) X_{1}(\theta, \varphi)$. The function $k(\varphi)$ is the ${ }^{106}$ Garren, D. A., and Boozer, A. H. (1991). Phys. Fluids B: Plasma Physics 3, 2805. 
reciprocal of the magnitude of the local radius of curvature of the magnetic axis. Clearly, $k(\varphi)$ cannot be identically zero, since the magnetic axis is required to bend for some values of $\varphi$ for it to be a closed curve. Hence, the function $X_{1}(\theta, \varphi)$ must be exactly zero in order that the first order curvature term in the field strength vanish identically.

The product $\varepsilon X_{1}(\theta, \varphi)$ is the first order term in the power series expansion of $X(\psi, \theta, \varphi)$ (see Fig. 5), defined in Eqs. (4.30) and (4.31). This lowest order expression for $X(\psi, \theta, \varphi)$ corresponds to $x(\psi, \theta, \varphi)$ of Eq. (1.4). The form of the function $X_{1}(\theta, \varphi)$ given in Eq. (4.81) is exactly zero if and only if the function $\eta(\varphi)$ completely vanishes, assuming nọn-zero axis field strength, $B_{0}(\varphi)$. However, the lowest-order flux surfaces collapse to ribbons that are both infinitely thin and and infinitely tall in the singular limit that $\eta(\varphi) \rightarrow 0$, with $B_{0}(\varphi) \neq 0$, as revealed in Sec. IV-E-2. Therefore, the first order variation in the magnetic field strength cannot be made to perfectly vanish ${ }^{107}$ for all values of the toroidal angle, $\varphi$. This result applies to any toroidal plasma equilibrium with non-trivial magnetic flux surfaces and non-vanishing magnetic field strength.

\section{B. Non-Arbitrariness through Third Order}

The magnetic field strength of toroidal plasma equilibria has the general form of Eq. (1.3). We will demonstrate that the $\varphi$ periodic functions $B_{0}(\varphi), \rho_{1}(\varphi), \lambda_{1}(\varphi), W_{0}(\varphi), F_{2}(\varphi), \delta_{2}(\varphi), C_{1}(\varphi)$, $\gamma_{1}(\varphi), c_{3}(\varphi)$, and $\gamma_{3}(\varphi)$ of Eqs. (1.3)-(1.6) cannot all be chosen ${ }^{107}$ Garren, D. A., and Boozer, A. H. (1991). Phys. Fluids B: Plasma Physics 3, 2805. 
freely. That is, the magnetic field strength of Eq. (1.3) is not arbitrary through third order in the expansion ${ }^{108}$, even if the first order curvature term, $k(\varphi) \times(\psi, \theta, \varphi)$, is guaranteed to be non-zero, in accord with the previous subsection. We now present the explicit procedure for extracting this result.

The magnetic field strength corresponding to a general toroidal plasma equilibrium can be expressed as

$$
\begin{aligned}
B(\psi, \theta, \varphi)=B_{0}(\varphi)\{1 & +\varepsilon\left\{b_{1.1 s}(\varphi) \sin (\theta)+b_{1.1 c}(\varphi) \cos (\theta)\right\} \\
+ & \varepsilon^{2}\left\{b_{2.0}(\varphi)+b_{2.2 s}(\varphi) \sin (2 \theta)+b_{2.2 c}(\varphi) \cos (2 \theta)\right\} \\
+ & \varepsilon^{3}\left\{b_{3.1 s}(\varphi) \sin (\theta)+b_{3.1 c}(\varphi) \cos (\theta)\right. \\
& \left.+b_{3.3 s}(\varphi) \sin (3 \theta)+b_{3.3 c}(\varphi) \cos (3 \theta)\right\} \\
& +\cdots\}
\end{aligned}
$$

using the form of analytic expansions presented within Sec. IV-C. Suppose that we would like to construct a toroidal plasma equilibrium with a particular form of the magnetic field strength through some order, say $\varepsilon^{j}$. That is, we seek a magnetic configuration corresponding to some arbitrary choice of the $\varphi$ periodic functions within the magnetic field strength of Eq. (5.1) through $\varepsilon^{j}$ order. The free selection of each of these functions of $\varphi$ is equivalent to imposing one independent constraint on the functions of $\varphi$ which determine the actual magnetic configuration. These constraints arising from the choice of the magnetic field strength must be applied in addition to those which guarantee the ${ }^{108}$ Garren, D. A., and Boozer, A. H. (1991). Phys. Fluids B: Plasma Physics 3, 2805. 
existence of equilibria through arbitrarily high order, which were developed within Chapter IV.

In Secs. IV-(E-G)-2, we showed that the first order set of matching constraints uniquely determine the magnetic field strength through $\varepsilon^{1}$ order. Similarly, the first through the $j \frac{\text { th }}{\text { sets of }}$ matching constraints determine the field strength through $\varepsilon^{j}$ order. This result follows since the highest order function that enters into the magnetic field strength of Eq. (4.97) through $\varepsilon^{j}$ order is the $X_{j}(\theta, \varphi)$ function of the $2(d \ell / d \varphi)^{2} k(\varphi) X_{j}(\theta, \varphi)$ term. The $X_{j}(\theta, \varphi)$ function is determined by the jth set of matching constraints. All other functions that enter the magnetic field strength through $\varepsilon^{j}$ order are determined by lower order sets of matching constraints. Hence, the jth and lower order sets of matching constraints uniquely determine the magnetic field strength through $\varepsilon^{j}$ order. This result is not as transparent if some form of the magnetic field strength other than that of Eq. (4.17) is implemented.

Assume that a magnetic configuration has been constructed through $\varepsilon^{\mathrm{j}-1}$ order. Correspondingly, the magnetic field strength is also determined through $\varepsilon^{j-1}$ order. Eq. (5.1) clearly shows that an arbitrary choice of the $\varepsilon^{J}$ order term of the magnetic field strength involves $j+1$ functions of $\varphi$. Thus, $j+1$ independent constraints must be satisfied by the functions of $\varphi$ that first enter the configuration at $\varepsilon^{j}$ order. Using the methodolgy of constructing toroidal plasma equilibria given within Secs. IV-(E-G), the $3 j+2$ independent $\varphi$ equations of the jth order set of matching constraints must also 
hold. Hence, a grand total of $4 j+3$ independent $\varphi$-constraints must be satisfied in constructing torodial equilibria with arbitrary forms of the magnetic field strength at $\varepsilon^{j}$ order, assuming these equilibria already exist through $\varepsilon^{j-1}$ order. The functions that are available to fulfill these constraints are the $3 j+4$ functions of $\varphi$ within the poloidal expansions of $X(\psi, \theta, \varphi), Y(\psi, \theta, \varphi)$, and $Z(\psi, \theta, \varphi)$, given within Eqs. (4.31)-(4.34). Obviously, the leading order of the new constraints that enter at a particular order, which scales as $4 j$, will eventually overtake the $3 j$ scaling of the new functions of $\varphi$ that enter. Therefore, the generic freedom in the expansion of the magnetic field strength will be broken at some particular order in the expansion. We prove that this loss of freedom occurs at third order.

The functions of $\varphi$ available to satisfy the various constraint equations are not all contained within the functions $X(\psi, \theta, \varphi)$, $Y(\psi, \theta, \varphi)$, and $Z(\psi, \theta, \varphi)$. The arbitrariness of the magnetic axis implies that the curvature, $x(\varphi)$, and torsion, $\tau(\varphi)$, functions introduce additional freedom into the system of equations. The only constraint on these two functions is that they must be consistent with a smooth, closed curve. Only two Fourier harmonics within a set of $K(l)$ and $\tau(l)$ need be varied in computational optimizations to obtain a smooth, closed curve, as was shown in Sec. III-C.

We show the breakdown in the freedom of the magnetic field strength by an eventual loss of free functions of $\varphi$ as the expansion is taken to higher order. Thus, we will assume, incorrectly in a 
strict sense, that $K(\varphi)$ and $\tau(\varphi)$ are entirely free functions of $\varphi$. This presumed freedom of the curvature and torsion functions can be used to help satisfy the $\varphi$-constraints in constructing toroidal equilibria with desired magnetic field strengths. In addition, this assumed freedom of $K(\varphi)$ and $\tau(\varphi)$ does not affect the 3 j scaling of the functions of $\varphi$ available to satisfy the $\varphi$-constraints at $\varepsilon^{j}$ order. We now examine the specific constraints and functions of $\varphi$ involved at each order in the expansion.

\section{V-B-1. Zeroth Order}

Only one constraint is implemented in the specification of the magnetic field strength at $\varepsilon^{0}$ order. This sole constraint arises from the freedom of the magnetic field strength on axis, $B_{0}(\varphi)$ of Eq. (5.1). The $\varphi$ dependent configuration functions available to satisfy this constraint are $\ell(\varphi), k[\ell(\varphi)]$, and $\tau[\ell(\varphi)]$, which completely determine the magnetic axis. Eq. (4.51) shows that the axis length, $l(\varphi)$, and the poloidal coil current, $G_{0}$, uniquely give the magnetic field strength of the axis, $B_{0}(\varphi)$. Thus, the functions $k(\varphi)$ and $\tau(\varphi)$ remain entirely arbitrary in the choice of the magnetic field strength of the axis. The presumed freedom of these two functions can be used to assist in the constraints of arbitrarily selecting the first order term of the magnetic field strength.

\section{V-B-2. First Order}

Two new constraints arise in the specification of the magnetic 
field strength at first order in $\varepsilon$, which result from the freedom of the functions $b_{1,1 s}(\varphi)$ and $b_{1,1 c}(\varphi)$ of Eq. (5.1). The $\varphi$ dependent configuration functions within the magnetic field strength of Eq. (4.97) are required to yield this desired form. In addition, these configuration functions must also satisfy the first order set of matching constraints that were defined within Secs. IV-(E-G). Thus, the entire set of constraints is given by: a) the two constraints that arise form the arbitrary specification of the functions $b_{1,1 s}(\varphi)$ and $b_{1,1 c}(\varphi)$ of the field strength in Eq. $\left.(5.1), b\right)$ the two $\hat{k}_{0}$ matching constraints at $\varepsilon^{1}$ order, c) the two $\hat{\tau}_{0}$ matching constraints at $\varepsilon^{1}$ order, and $d)$ the one $\hat{b}_{0}$ matching constraint at $\varepsilon^{0}$ order. The nine functions of $\varphi$ that are available to satisfy these seven constraints are specifically given by $X_{1,1 s}(\varphi), X_{1,1 c}(\varphi), Y_{1,1 s}(\varphi), Y_{1,1 c}(\varphi)$, $Z_{2,0}(\varphi), Z_{2,2 s}(\varphi)$, and $Z_{2.2 c}(\varphi)$ of Eqs. (4.31) and (4.32), and the residual zeroth order functions $k(\varphi)$ and $\tau(\varphi)$. Therefore, a maximum of two functions of $\varphi$ remain free after the magnetic field strength has been chosen through first order. The freedom of these two functions can be used within the analysis at second order.

\section{V-B-3. Second Order}

In choosing the second order term of the magnetic field strength, we must satisfy a) the three constraints that arise from the specification of the functions $b_{2,0}(\varphi), b_{2,2 s}(\varphi)$, and $b_{2,2 c}(\varphi)$ of the field strength of Eq. (5.1). Furthermore, we must also fulfill the second order matching constraints of Secs. IV-(E-G), which are 
explicitly given by: b) the three $\hat{K}_{0}$ matching constraints at $\varepsilon^{2}$ order, c) the three $\hat{z}_{0}$ matching constraints at $\varepsilon^{2}$ order, and d) the two $\hat{b}_{0}$ matching constraints at $\varepsilon^{1}$ order. The functions of $\varphi$ available to satisfy these constraints are $X_{2,0}(\varphi), X_{2,2 s}(\varphi), X_{2,2 c}(\varphi), Y_{2,0}(\varphi)$, $Y_{2,2 s}(\varphi), Y_{2,2 c}(\varphi), Z_{3,1 s}(\varphi), Z_{3,1 c}(\varphi), Z_{3,3 s}(\varphi)$, and $Z_{3,3 c}(\varphi)$ of Eqs. (4.32) and (4.33), in addition to the two functions of $\varphi$ that remain free after specification of the field strength through first order. This result yields a grand total of twelve free functions of $\varphi$ that are available for eleven independent constraints. Thus, after construction of the desired magnetic field strength through second order, a maximum of one free function of $\varphi$ is accessible to the constraints of third order.

\section{V-B-4. Third Order}

The constraints that enter in specifying the third order term of the magnetic field strength are: a) the four constraints arising from the choice of the functions $b_{3.1 \mathrm{~s}}(\varphi), b_{3.1 \mathrm{c}}(\varphi), b_{3.3 \mathrm{~s}}(\varphi)$, and $b_{3.3 c}(\varphi)$ of Eq. $\left.(5.1), b\right)$ the four $\hat{k}_{0}$ matching constraints at $\varepsilon^{3}$ order, b) the four $\hat{\varepsilon}_{0}$ matching constraints at $\varepsilon^{3}$ order, and $c$ ) the three $\hat{b}_{0}$ matching constraints at $\varepsilon^{2}$ order. These fifteen independent constraints must be satisfied by the thirteen functions $X_{3.1 \mathrm{~s}}(\varphi)$, $X_{3.1 c}(\varphi), X_{3,3 s}(\varphi), X_{3,3 c}(\varphi), Y_{3,1 s}(\varphi), Y_{3.1 c}(\varphi), Y_{3,3 s}(\varphi), Y_{3,3 c}(\varphi)$, $z_{4,0}(\varphi), z_{4,2 s}(\varphi), z_{4,2 c}(\varphi), Z_{4,4 s}(\varphi)$, and $z_{4,4 c}(\varphi)$, plus a maximum of one additional function of $\varphi$ not constrained through second order. Thus, a maximum of fourteen functions of $\varphi$ are accessible to the 
fifteen independent constraints, yielding an overdetermined system of equations. Therefore, we do not have generalized freedom in the specification of the magnetic field strength through third order in the expansion.

\section{IV-C. Field Strength Freedom Through Second Order}

In the previous section, we proved that the magnetic field strength corresponding to toroidal plasma equilibria is not arbitrary through third order. In this section, we show that there exists sufficient freedom to permit arbitrary forms of the magnetic field strength through second order ${ }^{109}$. The only provision is the nonvanishing of the zeroth and first order terms of the magnetic field strength. More specifically, torodial plasma equilibria can be constructed with desired forms for the functions $B_{0}(\varphi), \rho_{1}(\varphi), \lambda_{1}(\varphi)$, $W_{0}(\varphi), F_{2}(\varphi)$, and $\delta_{2}(\varphi)$ of the magnetic field strength of Eqs. (1.3)(1.5), provided that neither $B_{0}(\varphi)$, nor $\rho_{1}(\varphi)$, vanish for any value of $\varphi$.

The demonstration of the freedom in the magnetic field strength through second order follows in a manner similar to that of the restriction in the field strength through third order. However, this result regarding the field strength freedom is different from that of the previous section in one major respect. To prove the restriction in the magnetic field strength through third order, we assumed the maximal freedom of the magnetic axis. That is, the curvature, $K(\varphi)$, and torsion, $\tau(\varphi)$, functions were taken to be ${ }^{109}$ Garren, D. A., and Boozer, A. H. (1991). Phys. Fluids B: Plasma Physics 3, 2805. 
completely free functions of $\varphi$. This presumption followed from the analysis of Sec. III-D, which proved that only two Fourier harmonics within a set of $x(l)$ and $\tau(l)$ need be varied in optimizations to obtain a smooth, closed magnetic axis.

To show the freedom in the magnetic field strength through second order, we must assume the minimal possible freedom of the magnetic axis functions, $k(l)$ and $\tau(l)$. Clearly, we can presume that one these two functions is completely arbitrary, since only two Fourier harmonics within the set of $k(l)$ and $\tau(\ell)$ are required to serve as variable parameters in obtaining smooth, closed axes. The detailed analysis of the previous section for satisfying the matching constraints and the desired form of the magnetic field strength can then be refashioned to give the result of this section, using only one free function of $\varphi$ within $k[\ell(\varphi)]$ and $z[\ell(\varphi)]$, instead of two. The uniqueness of the Frenet representation of the spatial position for a given toroidal equilibrium implies that the $\varphi$ dependent configuration functions within this analysis contain no trivial freedoms resulting from only trivial translations and rotations of the coordinate system, as shown in Sec. IV-B. Furthermore, since non-zero plasma current and pressure do not affect the basic methodology of satisfying the matching constraints, as revealed within Secs. IV-(F-G), this result of the freedom in the magnetic field strength through second order holds for general toroidal plasma equilibria.

Detailed analysis also shows that only one of the two poloidal 
harmonics in the magnetic field strength at third order is free ${ }^{110}$, given arbitary forms of the field strength through second order. That is, either the $M=1$ poloidal harmonic functions $C_{1}(\varphi)$ and $\gamma_{1}(\varphi)$ of Eq. (1.6) or the $M=3$ poloidal harmonic functions $C_{3}(\varphi)$ and $\gamma_{3}(\varphi)$ are arbitrary, in addition to the lower order functions $B_{0}(\varphi), \rho_{1}(\varphi)$, $\lambda_{1}(\varphi), W_{0}(\varphi), F_{2}(\varphi)$, and $\delta_{2}(\varphi)$ within the field strength of Eqs. (1.3)(1.5). This result follows since two new functions of $\varphi$ remain free after the matching constraints at each particular order have been applied, as demonstrated in Sec. IV-(E-G)-3. In a related fashion, one poloidal harmonic within the magnetic field strength at any order can be chosen at whim. However, the other poloidal harmonics within the field strength at this particular order would not be arbitrary.

\section{V-D. Freedom on One Particular Flux Surface}

We have shown that the magnetic field strength corresponding to a toroidal plasma equilibrium cannot be chosen freely throughout the three-dimensional volume in the vicinity of the magnetic axis. This result is merely a reiteration of the non-arbitrariness of the magnetic field strength through third order, which was proven in Sec. V-B. However, the magnetic field strength is not prohibited from being specified freely on a two-dimensional flux surface. In fact, we find that the magnetic field strength on one particular magnetic flux surface is completely free ${ }^{111}$, provided that the first 110 Garren, D. A., and Boozer, A. H. (1991). Phys. Fluids B: Plasma Physics 3, 2805. 111 ibid. 
order curvature term does not entirely vanish.

To comprehend the freedom of the magnetic field strength on one flux surface, consider the general form of the field strength within any flux surface,

$$
B(\psi, \theta, \varphi)=B_{0}(\varphi)+\sum_{m=1}^{\infty} \varepsilon^{m}\left\{b_{m, s}(\varphi) \sin (m \theta)+b_{m, c}(\varphi) \cos (m \theta)\right\} .
$$

This equation is consistent with the form of analytic functions derived within IV-C. The particular magnetic flux surface depends on the value of $\varepsilon$, which now takes the role of a parameter rather than a variable. Recall from Secs. IV-(E-G)-3 that each order in the general equilibrium constraints introduces two functions of $\varphi$ that can be used to give some freedom in the specification of the configuration. Arbitrariness of these two functions of $\varphi$ at $j$ th order permits one to choose the functions $b_{j, s}(\varphi)$ and $b_{j, c}(\varphi)$ of Eq. (5.2) to give a desired form for the $\varepsilon^{j}$ order term of the magnetic field strength. Since this selection applies for all orders in $\varepsilon$, the magnetic field strength on one flux surface can be chosen entirely freely. The only restriction is that the zeroth and first order terms must not completely vanish, in accord with Sec. V-A. This result is fully general for any toroidal, scalar-pressure plasma equilibrium. Hence, the confinement properties can be optimized more freely near one particular magnetic flux surface than throughout a threedimensional volume. 


\section{CHAPTER V1 \\ QUASI-HELICALLY SYMMETRIC EQUILIBRIA}

The expression for the magnetic field strength in terms of Boozer coordinates largely determines 112 the confinement and stability properties of a toroidal plasma equilibrium, as demonstrated within Chapter II. Furthermore, widely differing configurations with similar forms for the magnetic field strength in terms of Boozer coordinates were shown to have related plasma transport properties ${ }^{113}$. However, toroidal equilibria corresponding to desirable forms of the magnetic field strength may not always exist. Specifically, the Taylor-Fourier series expansion of the magnetic field strength has restrictions 114 that arise from the toroidicity of the configuration and the divergence-free property of the magnetic field, as proven within the previous chapter.

In this chapter, we will implement the generic restrictions and freedoms of the magnetic field strength to investigate particularly significant types of toroidal plasma equilibria. The most desirable class of toroidal plasma equilibria exhibits constant magnetic field strength within the plasma pressure surfaces, as first proposed by Palumbo115. However, such toroidal "isodynamic" 112 Boozer, A. H. (1984). Phys. Fluids 27, 2441. 113 Boozer, A. H. (1983). Phys. Fluids 26, 496.

${ }^{114}$ Garren, D. A., and Boozer, A. H. (1991). Phys. Fluids B: Plasma Physics 3, 2805. 
equilibria can exist only in limits in which magnetic field strength on axis vanishes or the magnetic flux surfaces become open, as proven by Bernardin, Moses, and Tataronis ${ }^{116}$. Thus, these investigators conclude that toroidal isodynamic equilibria are not practical for applications of constructing a fusion reactor. In the first section of this chapter, we will demonstrate the first order symmetry breaking of toroidal isodynamic equilibria within the framework of our analysis.

The next most desirable class of toroidal plasma equilibria is characterized by a magnetic field strength which depends on only one angular coordinate within the constant pressure surfaces. Such toroidal equilibria are said to be "quasi-helically symmetric"117. In the second section of this chapter, we show that quasi-helical symmetry is always broken at third order ${ }^{118}$, i.e., $(\Delta B) / B \approx \varepsilon^{3}$. Nevertheless, good approximations of quasi-helical symmetry exist for practical values of $\varepsilon \approx 1 / 10$, since the symmetry breaking occurs at third order, rather than some lower order. The remaining sections of this chapter are devoted to developing generic properties and sample configurations of toroidal plasma equilibria which highly approximate quasi-helical symmetry.

115 Palumbo, D. (1968). I/ Nuovo Cimento X53B, 507.

116 Bernardin, M. P., Moses, R. W., and Tetaronis, J. A. (1986). Phys. Fluids 29, 2605.

117 Nührenberg, J., and Zille, R. (1988). Phys. Lett. A. 129, 113.

${ }^{118}$ Garren, D. A., and Boozer, A. H. (1991). Phys. Fluids B: Plasma Physics 3, 2822. 


\section{VI-A. Breaking of Toroidal Isodynamic Equilibria}

The magnetic field strength of isodynamic plasma equilibria is defined to be uniform within surfaces of constant pressure. Hence, the magnetic field strength has the generic form $B(\psi)$ in terms of Boozer coordinates. As first noted by Palumbot19, such equilibria are highly desirable, since their guiding-center particle trajectories remain within the pressure surfaces for all time: These favorable particle trajectories arise from the conservation of the canonical momenta $P_{\theta}$ and $P_{\varphi}$ using Eq. (2.30), which follows from the absence of the coordinates $\theta$ and $\varphi$ from the magnetic field strength of the drift Hamiltonian in Eq. (2.26). Because the guiding-center trajectories do not cross the pressure surfaces, plasma transport across the pressure surfaces due to guiding-center drift motion does not exist. However, transport due to classical diffusion remains, although it is weaker than guiding-center transport by the ratio of the particle gyro-radius to the radius of curvature of the magnetic field lines. This ratio is approximately $1 / 500$ for plasmas of fusion interest.

Bernardin, Moses, and Tataronis 120 have performed a detailed investigation of isodynamic equilibria. They have proven that toroidal isodynamic equilibria exist only in two limits: 1) the magnetic flux surfaces become open, and 2) the magnetic field strength of the magnetic axis vanishes. We will now use the 119 Palumbo, D. (1968). II Nuovo Clmento X53B, 507.

${ }^{120}$ Bernardin, M. P., Moses, R. W., and Tetaronis, J. A. (1986). Phys. Fluids 29, 2605. 
formalism developed within this dissertation to demonstrate these two limits through first order in an expansion about a magnetic axis.

VI-A-1. First Limit: Collapsing Magnetic Flux Surfaces

The magnetic field strength corresponding to toroidal MHD equilibria through first order is given by Eq. (4.71). This form of the field strength applies regardless of the plasma current and pressure profiles, as shown within Secs. IV-(F-G). The expression for the magnetic field strength in terms of Boozer coordinates, Eq. (4.71), must have the form $B(\psi)$ in order to be consistent with torodial isodynamic equilibria.

The magnetic field strength on axis, $B_{0}(\varphi)$ of Eq. (4.71), can obviously be made to be independent of $\varphi$. Thus, toroidal isodynamic equilibria trivially exist through $\varepsilon^{0}$ order. However, obstacles arise in constructing toroidal isodynamic equilibria through first order.

The first order term in the magnetic field strength of Eq. (4.71) is given by $B_{0} K(\varphi) X_{1}(\theta, \varphi)$, with $B_{0}$ constant Assuming nonvanishing axis field strength, the product $k(\varphi) X_{1}(\theta, \varphi)$ is required to be independent of the angles $\theta$ and $\varphi$ in order to give toroidal isodynamic eqilibria. The function $X_{1}(\theta, \varphi)$ always exhibits nontrivial $\theta$ dependence, presuming the function $\eta(\varphi)$ of Eq. (4.81) is non-zero. In the singular limit in which the function $\eta(\varphi)$ does vanish, the lowest order flux surfaces collapse to ribbons that are both infinitely thin and infinitely tall, as developed within Sec. I $\forall$ E-2-b. Since the axis curvature, $k(\varphi)$, cannot completely vanish for toroidal configurations, the first order term of the magnetic field 
strength is compelled to have non-trivial $\theta$ dependence, assuming the magnetic field strength of the axis is non-zero.

One obvious limit in which toroidal isodynamic equilibria exist corresponds to the vanishing of the function $\eta(\varphi)$ of Eq. (4.81). For this case, the magnetic flux surfaces collapse to ribbons that are both infinitely thin and infinitely tall near the magnetic axis. That is, the flux surfaces are flattened along the local curvature vector of the magnetic axis, $\hat{k}_{0}(l)$ of Fig. 5. This limit of toroidal isodynamic equibria corresponds to the magnetic flux surfaces becoming open, as first demonstrated by Bernardin, Moses, and Tataronis $^{121}$.

\section{VI-A-2. Second Limit: Vanishing Axis Field Strength}

Palumbo122 analytically constructed a particular class of axisymmetric isodynamic equilibria. These toroidal plasma equilibria are characterized by zero magnetic field strength on the magnetic axis. Thus, the local plasma beta, which is defined to be the ratio of the plasma energy density to that of the magnetic field, i.e., $\beta \equiv 2 \mu_{0} p / B^{2}$, is infinite on the magnetic axis. As expected, he found that this class of toroidal isodynamic equilibria was highly unstable to pressure-driven perturbations.

The near-axis results of Palumbo equilibria can be obtained through observation of Eq. (4.80) for the magnetic field strength of

${ }^{121}$ Bernardin, M. P., Moses, R. W., and Tetaronis, J. A. (1986). Phys. Fluids 29, 2605.

122 Palumbo, D. (1968). II Nuovo Cimento X53B, 507. 
general MHD equilibria through first order. The vanishing of the axis field strength, $B_{0}(\varphi)$ in Eq. (4.80), clearly eliminates the zeroth and first order terms within the magnetic field strength. This result can be achieved using non-trivial values for the curvature, $k(\varphi)$, and ellipticity along the curvature, $\eta(\varphi)$, of Eq. (4.80). Since the explicit $\theta$ and $\varphi$ dependence has been entirely vanquished from the field strength through first order, toroidal isodynamic equilibria exist at least through first order, in this limit.

The vanishing of the magnetic field strength on axis implies that zero net poloidal electric current flows through the closed curve comprising the magnetic axis. Thus, the poloidal diamagnetic current within the plasma must totally offset the poloidal current in the field coils, in order that the axis field strength completely vanish. An extremely large volume-averaged, plasma beta $\langle\beta\rangle \approx 1$ is required to make the magnetic field strength of the axis approach zero. Hence, this class of toroidal isodynamic equilibria is expected to be exceedingly unstable to pressure-driven perturbations.

These two limits for attaining toroidal isodynamic equilibria, i.e., the flattening of the magnetic flux surfaces and the vanishing of the axis field strength, are independent. One can obviously construct low beta equilibria in which the flux surfaces are highly flattened along the curvature vector. One can also construct high beta equilibria with near-circular magnetic flux surfaces. 


\section{VI-B: Breaking of Quasi-Helical Symmetry}

In the previous section, terms of first order in $\varepsilon$ were shown to preclude the existence of exact toroidal isodynamic equilibria. Because the symmetry breaking occurs at first order, rather than some higher order, good approximations of toroidal isodynamic equilibria do not exist. Therefore, we attempt to find toroidal equilibria with the second most desirable plasma transport properties, which are those exhibiting quasi-helically symmetry.

The magnetic field strength corresponding to quasi-helically symmetric equilibria has the general form $B(\psi, \alpha)$, with $\alpha \equiv \theta-N \varphi$ a helical coordinate and $\mathrm{N}$ an integer, in terms of Boozer coordinates. This form of the magnetic field strength obviously exists for configurations which are perfectly axisymmetric, i.e. $\varphi$ independent, giving the trivial $\mathrm{N}=0$ case. Non-axisymmetic toroidal equilibria in which the magnetic field strength depends on only one angular coordinate within the flux surfaces were called "quasi-helically symmetric," by Nührenberg and Zille ${ }^{123}$. They computaionally found large aspect ratio stellarator equilibria which closely approximate quasi-helical symmetry.

The particle drift trajectories of quasi-helically symmetric stellarator equilibria would be similar 124 to those of axisymmetric tokamak equilibria. For example, the tips of a trapped banana orbit for both quasi-helically symmetric stellarators and axisymmetric tokamaks remain within one magnetic flux surface. That is, quasi${ }^{123}$ Nührenberg, J., and Zille, R. (1988). Phys. Lett. A. 129, 113. ${ }^{124}$ Boozer, A. H. (1983). Phys. Fluids 26, 496. 
helically symmetric stellarators would have a negligible number of super-banana125 orbits that lead to the enhanced transport of conventional stellarators.

The favorable drift trajectories of quasi-helically symmetric equilibria arise from the existence of a canonically conserved momentum, $\mathrm{P}_{h}$ of Eq. (2.48), that is analogous to the $\mathrm{P}_{\varphi}$ invariant of axisymmetric tokamak equilibria. The invariance of $\mathrm{P}_{h}$ was proven in Sec. II-D.

The magnetic field strength corresponding to quasi-helically symmetric plasma equilibria must satisfy the constraint

$$
\|B(\psi, \theta, \varphi)\|=B(\psi, \alpha) .
$$

with $\alpha \equiv \theta-N \varphi$ the helical coordinate and $N$ any integer. Such a constraint of forcing a general function of three variables to be a function of only two is obviously independent of the general equilibrium constraints developed within Sec. IV-(E-G). In addition, the quasi-helical constraint of Eq. (6.1) does not introduce any free functions of position into the system of equations.

We attempt to find quasi-helically symmetric equilibria through all orders in the expansion about a magnetic axis. The magnetic field strength of a quasi-helically symmetric stellarator has the general form

${ }^{125}$ Beidler, C., et. al. (1990). Fusion Technology: A Journal of the American Nuclear Society 17, 148. 


$$
\begin{aligned}
B(\psi, \alpha)=B_{0}\{1 & +\varepsilon\left\{h_{1,1 s} \sin (\alpha)+h_{1,1 c} \cos (\alpha)\right\} \\
& +\varepsilon^{2}\left\{h_{2,0}+h_{2,2 s} \sin (2 \alpha)+h_{2,2 c} \cos (2 \alpha)\right\} \\
& +\varepsilon^{3}\left\{h_{3,1 s} \sin (\alpha)+h_{3,1 c} \cos (\alpha)\right. \\
& \left.\quad+h_{3,3 s} \sin (3 \alpha)+h_{3,3 c} \cos (3 \alpha)\right\} \\
& +\cdots\}
\end{aligned}
$$

with the Fourier coefficients $B_{0}, h_{1,1 s}, h_{1,1 c}, h_{2,0}, h_{2,2 s}$, etc., all constant. We apply the results of Sec. V-B regarding the generic restriction in the expansion of the magnetic field strength through third order. This analysis implies that one or more of the Fourier coefficients $B_{0}, h_{1,1 s}, h_{1,1 c}, h_{2,0}, h_{2,2 s}, h_{2,2 c}, h_{3,1 s}, h_{3,1 c}, h_{3,3 s}$, and $h_{3.3 c}$ of Eq. (6.2) must exhibit non-trivial $\varphi$ dependence. Hence, quasi-helically symmetric plasma equilibria do not exist ${ }^{126}$ through through third order in $\varepsilon$.

We also deduce that quasi-helical symmetry is broken by terms of second order in $\varepsilon$ for toroidal plasma equilibria constrained to have circular magnetic axes ${ }^{127}$. This result is obtained in a manner similar to that for the third order symmetry breaking of toroidal equilibria with unconstrained magnetic axes. The only modification of the analysis for the circular axis case is that the axis curvature, $k(\varphi)$, must be constant, and the axis torsion, $\tau(\varphi)$, must be zero. Thus, these two magnetic axis functions are no longer available to assist in satisfying the various $\varphi$-constraints involved in constructing quasi-helically symmetric equilibria.

${ }^{126}$ Garren, D. A., and Boozer, A. H. (1991). Phys. Fluids B: Plasma Physics 3, 2822. 127 Ibid. 


\section{VI-C. Properties of Approximate Quasi-Helical Symmetry}

Good approximations to quasi-helical symmetry can be constructed, since the symmetry breaking scales as third order in the expansion parameter, $\varepsilon$. We obtain the following characteristics of plasma equilibria that accurately approximate quasi-helical symmetry ${ }^{128}$ :

1. Non-Zero Curvature of the Magnetic Axis

The curvature of the magnetic axis, $k(\varphi)$, must never vanish for plasma equilibria which are quasi-helically symmetric through first order in $\varepsilon$. A demonstration of this result will be derived within the SeC. VI-D.

2. Non-Zero Torsion of the Magnetic Axis

The torsion of the magnetic axis, $\tau(\varphi)$, must be non-zero, since quasi-helical symmetry is broken at second order for circular-axis configurations, but only at third order for configurations with nonzero torsion. In addition, circular-axis equilibria which approximate quasi-helical symmetry and have zero net toroidal current are constrained to have zero rotational transform on axis, $L_{0}$, as we will demonstrate in Sec. VI-D. An equilibrium which approximates quasi-helical symmetry and has a helical-like axis and vanishing net toroidal current is not required to have a vanishing axis transform. A non-zero rotationat transform on axłs ${ }^{128}$ Garren, D. A., and Boozer, A. H. (1991). Phys. Fluids B: Plasma Physics 3, 2822 
is beneficial since it stabilizes a plasma equilibrium against certain pressure-driven perturbations ${ }^{129}$.

\section{Small Toroidal Variation of the Magnetic Flux Surface Shape}

Quasi-helically symmetric equilibria are obtained in the limit in which the functions $X(\psi, \theta, \varphi), Y(\psi, \theta, \varphi)$, and $Z(\psi, \theta, \varphi)$ of the spatial position of Eq. (4.18) all become independent of the toroidal angle, $\varphi$. This result follows from the fact that a given number of independent $\varphi$-equations can be satisfied by a fewer number of functions of $\varphi$ if all of the functions of $\varphi$ are chosen to be constants. The expansion coefficients within the flux functions $G(\psi), I(\psi), L(\psi)$, and $p(\psi)$ provide the additional constants necessary to satisfy this system of quasi-helical equilibrium equations. However, if the functions $X(\psi, \theta, \varphi), Y(\psi, \theta, \varphi)$, and $Z(\psi, \theta, \varphi)$ are all required to be independent of $\varphi$, then the axis curvature, $K(\varphi)$, and torsion, $\tau(\varphi)$, must be constants. Integration of the Frenet equations of Eqs. (3.1)-(3.4) reveals that any curve with constant, non-vanishing torsion and curvature forms a straight helix, and thus cannot form a closed curve. The choice of zero torsion and constant, non-zero curvature merely reduces the equilibium to the trivial axisymmetric case.

Small fractional $\varphi$-variation of the curvature and torsion of the magnetic axis gives a better approximation to quasi-helical symmetry. The beating of $\varphi$-harmonics within the non-linear equations involved is not conducive to accidental proximity of

129Bateman, G. (1978). MHD Instabilities, MIT Press, Cambridge, Massachusetts, and London, England, p. 64. 
quasi-helical symmetry for large fractional $\varphi$-variation. Thus, if $f(\varphi)$ is some appropriate measure of the fractional toroidal variation of the magnetic flux surfaces, then quasi-helical symmetry is broken by terms of order $f(\varphi) \varepsilon^{2}$ for circular axis configurations, and by terms of order $f(\varphi) \varepsilon^{3}$ for configurations with unconstrained axes.

Quasi-helical symmetry is most accurately approximated by configurations with a large number of magnetic field periods $N$, so that the magnetic axis can approximate a straight helix locally. This result follows from the smaller fractional $\varphi$-variation of the curvature and torsion of the magnetic axis as the number of field periods is increased. Hence, the factor $f(\varphi)$ within the third order symmetry breaking term can be made to be relatively small for configurations with many field periods. Sample configurations that demonstrate these results will be presented in Sec. VI-D.

\section{Small Local Inverse Aspect Ratio}

The expansion parameter, $\varepsilon$, is roughly the quotient of the plasma minor radius, $r$, and the minimum local radius of curvature of the magnetic axis, $R_{c}$. Keeping this expansion parameter small, i.e., $\varepsilon \approx 1 / 10$ corresponding to the maximum pressure gradient, also minimizes the asymmetries in the magnetic field strength for configurations that highly approximate quasi-helical symmetry.

5) Quasi-Helical Symmetry on One Flux Surface

We have demonstrated that quasi-helical symmetry cannot be 
made exact within the three-dimensional volume in the vicinity of the magnetic axis for non-axisymmetric, toroidal plasma equilibria. However, this proof does not prohibit the existence of quasi-helical symmetry within one particular magnetic flux surface. In fact, the analysis of Sec. V-D permits the existence of plasma equilibria in which the magnetic field strength is a function of only one angular coordinate within one particular flux surface ${ }^{130}$, but not within its three dimensional interior. Such toroidal equilibria would have no plasma transport across this particular flux surface, to lowest order in the guiding-center analysis.

The magnetic field strength within a quasi-helically symmetric flux surface has the general form

$$
B\left(\alpha_{i} \varepsilon\right)=B_{0}+\sum_{m=1}^{\infty} \varepsilon^{m}\left\{h_{m, s} \sin (m \alpha)+h_{m, c} \cos (m \alpha)\right\}
$$

in terms of the helical angle $\alpha \equiv \theta-N \varphi$, with the axis field strength, $B_{0}$, and the Fourier coefficients $h_{m, s}$ and $h_{m, c}$ all constants. The relevant magnetic flux surface is determined by the particular choice of the parameter, $\varepsilon$. The analysis in Sec. V-D demonstrates that the magnetic field strength within one particular flux surface, Eq. (5.2), can be expresssed in the quasi-helical form of Eq. (6.3). The only requirement is that the first order term must be non-zero, so that $\left\{\left\{h_{1, s}\right\}^{2}+\left\{h_{1, c}\right\}^{2}\right\}^{1 / 2}$ is compelled to be a positive constant. ${ }^{130}$ Garren, D. A., and Boozer, A. H. (1991). Phys. Fluids B: Plasma Physics 3, 2822. 
We have shown that the magnetic field strength can be made to depend on only one angular coordinate within one particular magnetic flux surface, corresponding to quasi-helical symmetry on that flux surface. However, the three dimensional interior of this flux surface could be only approximately quasi-helically symmetric. In fact, the breaking of quasi-helical symmetry near the magnetic axis of such an equilibrium would scale ${ }^{131}$ as $\left\{\left(\varepsilon_{\mathrm{a}}\right)^{2}-\varepsilon^{2}\right\} \varepsilon$, rather than $\varepsilon^{3}$. This result follows since an additional first order term in the magnetic field strength that scales as $\left(\varepsilon_{a}\right)^{2} \varepsilon$ must be used to offset the $\varepsilon^{3}$ order symmetry-breaking term at the magnetic surface corresponding to $\varepsilon=\varepsilon_{\mathrm{a}}$. Hence, imposing quasi-helical symmetry on one particular flux surface worsens the approximation of quasihelical symmetry within its interior.

\section{VI-D. Examples of Approximate Quasi-Helical Symmetry}

Quasi-helical symmetry through zeroth order is characterized entirely by the magnetic axis itself. The shape of this magnetic field line is completely arbitrary, provided that it is a smooth, closed curve. The only additional constraint for quasi-helical symmetry through zeroth order is that the magnetic field strength of the axis must be independent of the toroidal angle, $\varphi$. Since samples of quasi-helical symmetry through zeroth order can be found trivially, we begin by constructing configurations which are quasi-helically symmetric through first order.

${ }^{131}$ Garren, D. A., and Boozer, A. H. (1991). Phys. Fluids B: Plasma Physics 3, 2822. 
VI-D-1. Examples of Quasi-Helical Symmetry through First Order Quasi-helically symmetric equilibria through first order are determined by the magnetic flux surfaces through first order. In order to generate these lowest order flux surfaces, an appropriate magnetic axis must first be chosen. The axis curvature, $K(\varphi)$, of a configuration which is quasi-helically symmetric through first order must never vanish, as we will demonstrate later in this section. We implement the parametric analysis of Sec. III-B to construct magnetic axes compatible with quasi-helical symmetry through first order. This procedure entails choosing the parameters $N, M$, and $\zeta$ within $r_{0}[\ell(\Phi)]$ of Eq. (3.9), so that the curvature function, $k(\Phi)$ of Eq. (3.10), never vanishes along the axis length.

Given an axis with non-vanishing curvature, the results of Sec. IV-E-2-b can be used to construct the lowest order, quasi-helical flux surfaces about the axis. The magnetic field strength corresponding to quasi-helical symmetry through first order has the form of Eq. (4.79), with $B_{0}(\varphi)$ and $\delta(\varphi)$ positive constants and $\alpha(\varphi)=N \varphi$. Use of Eq. (4.88) for $\delta(\varphi)$ implies that the function $\eta(\varphi)$ must have the form

$$
\eta(\varphi)=\bar{\eta} / k(\varphi),
$$

with $\bar{\eta}$ a positive constant. However, the function $\eta(\varphi)$ is required to be non-zero and non-infinite for all $\varphi$ in order that the lowest order flux surfaces not collapse to ribbons that are infinitely thin 
and infinitely tall, as revealed within Sec. IV-E-2-b. Hence, Eq. (6.4) demonstrates that the axis curvature, $k(\varphi)$, can never vanish for configurations which are quasi-helically symmetric through first order ${ }^{132}$. This result was the first generic condition given within Sec. VI-C for approximating quasi-helical symmetry.

In summary, given an axis with non-vanishing curvature, the flux surfaces corresponding to quasi-helical symmetry through first order can be obtained using $B_{0}(\varphi)=1, \eta(\varphi)=\bar{\eta} / k(\varphi)$, and $\alpha(\varphi)=N \varphi$ within Eqs. (4.79)-(4.83) of Sec. IV-E-2-b. Integration of $d \sigma / d \varphi$ in Eq. (4.83) from zero to $2 \pi$ reveals that the constant $\imath_{0}-N$ is bound between zero and

$$
U=\frac{2 G_{0} \int_{0}^{2 \pi}\{\eta(\varphi)\}^{2}\left\{\tau(\varphi)+(1 / 2) I_{2}\right\} d \varphi}{\int_{0}^{2 \pi}\left\{1+\{\eta(\varphi)\}^{4}\right\} d \varphi}
$$

for quasi-helical symmetry through first order. This restriction shows that the true transform on axis, $l_{0}-N$, vanishes for quasihelically symmetric equilibria with a circular magnetic axis and zero net toroidal current on axis, $l_{2}$. Higher order effects can give a global shear, $l_{2} / l_{0}$, to guarantee the existence of such equilibria with vanishing axis transform. However, toroidal equilibria with a vanishing rotational transform on axis are usually unstable to ${ }^{132}$ Garren, D. A., and Boozer, A. H. (1991). Phys. Fluids B: Plasma Physics 3, 2822 
certain pressure-driven perturbations ${ }^{133}$.

We implement Eqs. (6.4) and (4.80)-(4.83) to graph quasihelically symmetric flux surfaces through first order about any magnetic axis with non-vanishing curvature. The magnetic axis of our first example is given by Eq. (3.9) with $N=5, M=1$, and $\zeta=1 / 15$, which is the five-period helical curve of Figs. 1 and 6 . The constant $\bar{\eta}$ of Eq. (6.4) is determined by $\bar{\eta}=1.25 / \sqrt{2}$, so that the function $\delta(\varphi)$ within the field strength of Eq. $(4.79)$ is $\delta(\varphi)=1.25$. This choice yields elliptical flux surfaces which are rather flattened perpendicular to the curvature vector, resulting in a somewhat minimized first order variation of the magnetic field strength. A sample magnetic flux surface for this five-period helical configuration is given in Fig. 12. The rotational transform is determined by $L_{0}=3.673$, so that the largest value of $|\sigma(\varphi)|$, which gives a contribution to the ellipticity of the flux surfaces via Eq. (4.85), is minimized.

The rotational transform utilized within this dissertation is not the conventional rotational transform. Within this analysis, the $\theta=0$ position rotates with the curvature vector, $\hat{k}_{0}(\varphi)$, whereas, customarily, it does not. Our choice of the transform is transparent through observation of "knotted" configuration of Fig. 13. The conventional rotational transform $\iota_{\text {con }}$ is determined by $|\mathrm{l}-\mathrm{N}|$, with $\downarrow$ the transform of this analysis.

A ten period helical toroid exhibiting quasi-helical symmetry ${ }^{133}$ Bateman, G. (1978). MHD Instabilities, MIT Press, Cambridge, Massachusetts, and London, England, p. 64. 
through first order is introduced within Fig. 14. The parameters corresponding to this configuration are specified by $N=10, M=1$, and $\zeta=1 / 15$ within Eq. (3.9) for $r_{0}[\ell(\Phi)]$. The constant $\bar{\eta}$ was chosen to be $1.25 / \sqrt{2}^{\prime}$ in order to yield the same first order variation of the magnetic field strength for this ten-period helical toroid as for the one of five periods given in Fig. 12. The minimization of $|\sigma(\varphi)|$ leads to a transform on axis of $l_{0}=7.631$.

The normalized values for the axis curvature, $k(l)$, and torsion, $\tau(\ell)$, corresponding to these helical toroids is computed using Eqs. (3.10) and (3.11). These axis functions for the five and ten period cases are given by Figs. 6 and 15, respectively. These graphs demonstrate the smaller variation of the curvature and torsion with a larger number of field periods. However, these figures also indicate that the mean curvature increases with the number of field periods. Hence, there exists a compromise between the variation of the curvature and torsion functions, which determines the flattening of the quasi-helical flux surfaces, and the mean curvature, which dominates the guiding-center drift trajectories within the equilibrium.

Configurations other than those of conventional stellarators can yield quasi-helical symmetry through first order. One sample is the three-period knotted configuration of Fig. 13. The magnetic axis of this configuration is determined by $\{N=3, M=2, \zeta=1 / 2\}$ within Eq. (3.9), as in Figs. 7 and 8 . The corresponding magnetic field strength exhibits the same first order variation as that of the 


\section{FIGURE 14}

\section{TEN PERIOD HELICAL CONFIGURATION}

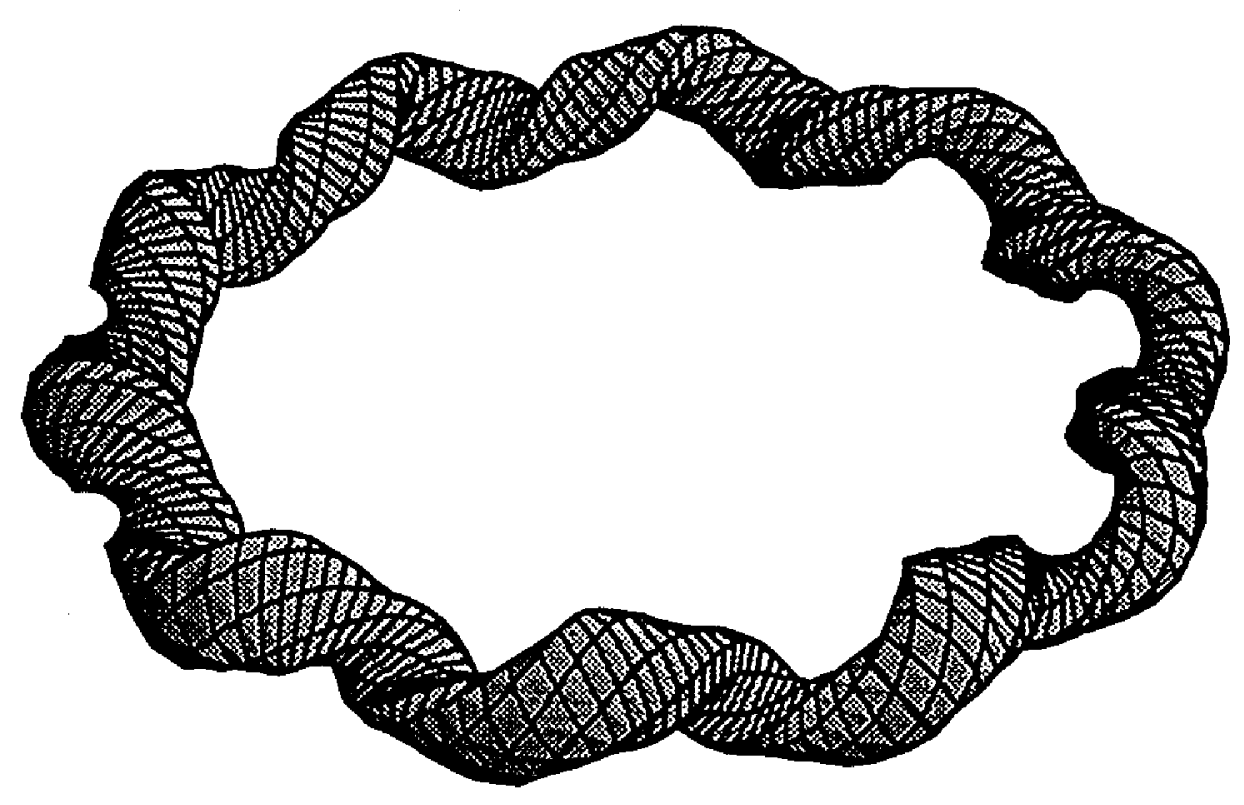

The lowest order flux surfaces of a ten-period helical configuration satisfying quasi-helical symmetry through first order. The magnetic axis is given by Eq. (3.9) with $N=10, M=1$, and $\zeta=1 / 15$. The magnetic field strength has the form of Eq. (4.79) with $\delta(\varphi)=1.25$, and the rotational transform on axis is $\iota_{0}=7.631$. 
FIGURE 15

CURVATURE AND TORSION OF TEN PERIOD HELICAL AXIS

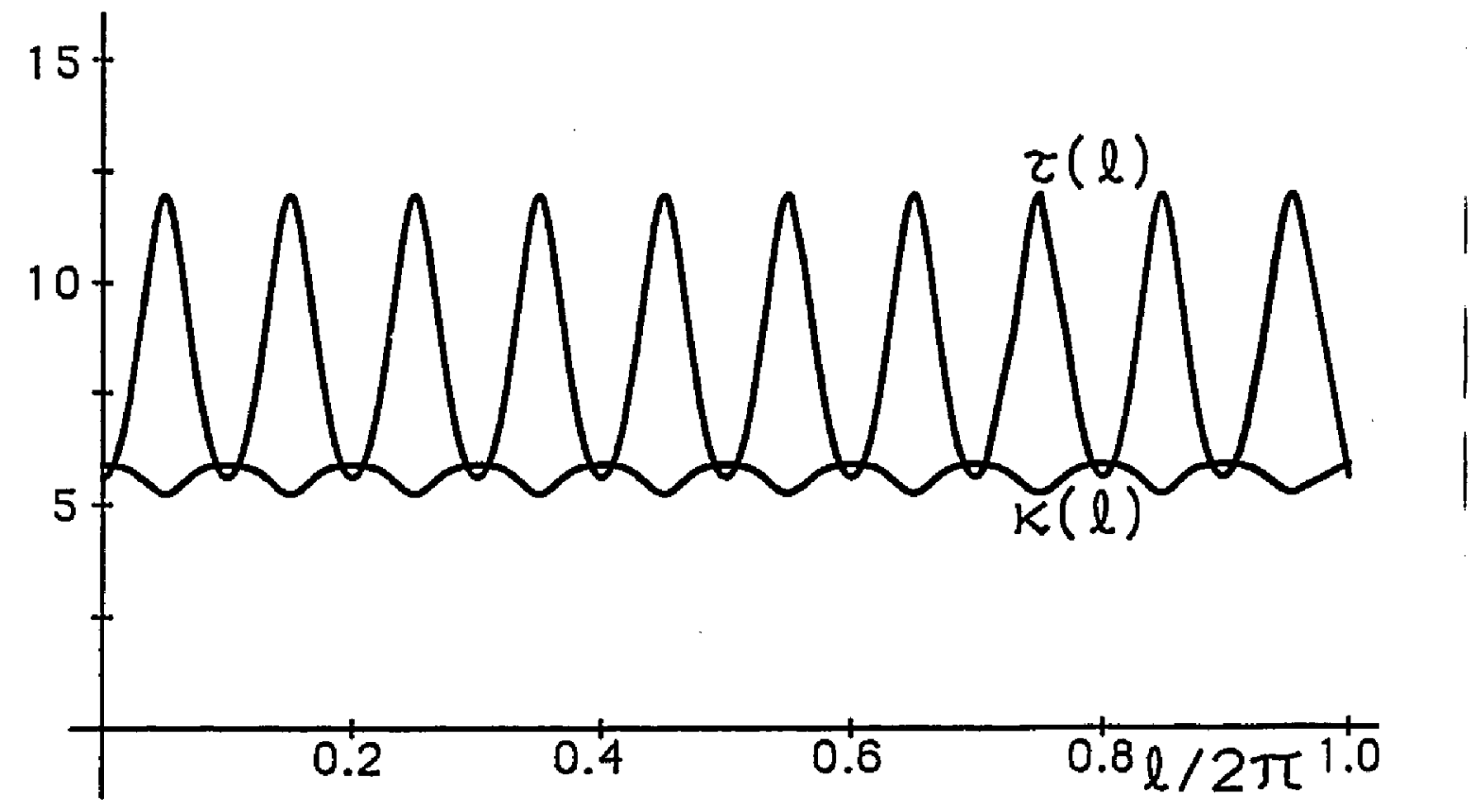

The curvature and torsion corresponding to the ten-period helical configuration of Fig. 14, assuming the magnetic axis has length $L=2 \pi$. 
helical configurations of Figs. 12 and 14. The value for $l_{0}$ is 0.991 , again chosen to minimize the maximum of $|\sigma(\varphi)|$.

Another sample of a three-period knot exhibiting quasi-helical symmetry through first order is shown in Figs. 16 and 17. The axis corresponding to this configuration is obtained using $\{N=3, \quad M=2$, $\zeta=3 / 4\}$ within Eq. (3.9). The value of the constant $\bar{\eta}$ of Eq. (6.4) is again chosen to be $1.25 / \sqrt{2}$, giving the same first order field strength variation as in the previous examples. Minimization of the largest value of $|\sigma(\varphi)|$ gives $\imath_{0}=0.565$. Unfortunately, the smoother curvature and torsion functions, which yield a smaller toroidal variation of the magnetic flux surface shape, correspond to the less practical, "tightly" knotted configuration of Fig. 16.

A five-period knot with quasi-helical symmetry through first order is presented in Figs. 18 and 19. Its configuration parameters are given by $\left\{N=5, \quad M=2, \zeta=1 / 2, \quad \bar{\eta}=1.25 / \sqrt{2}, \quad \iota_{0}=1.671\right\}$. The curvature and torsion corresponding to the magnetic axis of this example are relatively smooth, as seen in Fig. 19, giving the somewhat minimized toroidal variation in the shape of the magnetic flux surfaces of Fig. 18.

VI-D-2. Examples of Quasi-Helical Symmetry through Second Order In Sec. VI-B, we showed that quasi-helical symmetry does not exist through second order for circular axis configurations. The shape of the axis, via the curvature, $k[\ell(\varphi)]$, and torsion, $\tau[\ell(\varphi)]$ functions, must be optimized in order to obtain quasi-helical 


\section{"TIGHTER" THREE PERIOD KNOTTED CONFIGURATION}

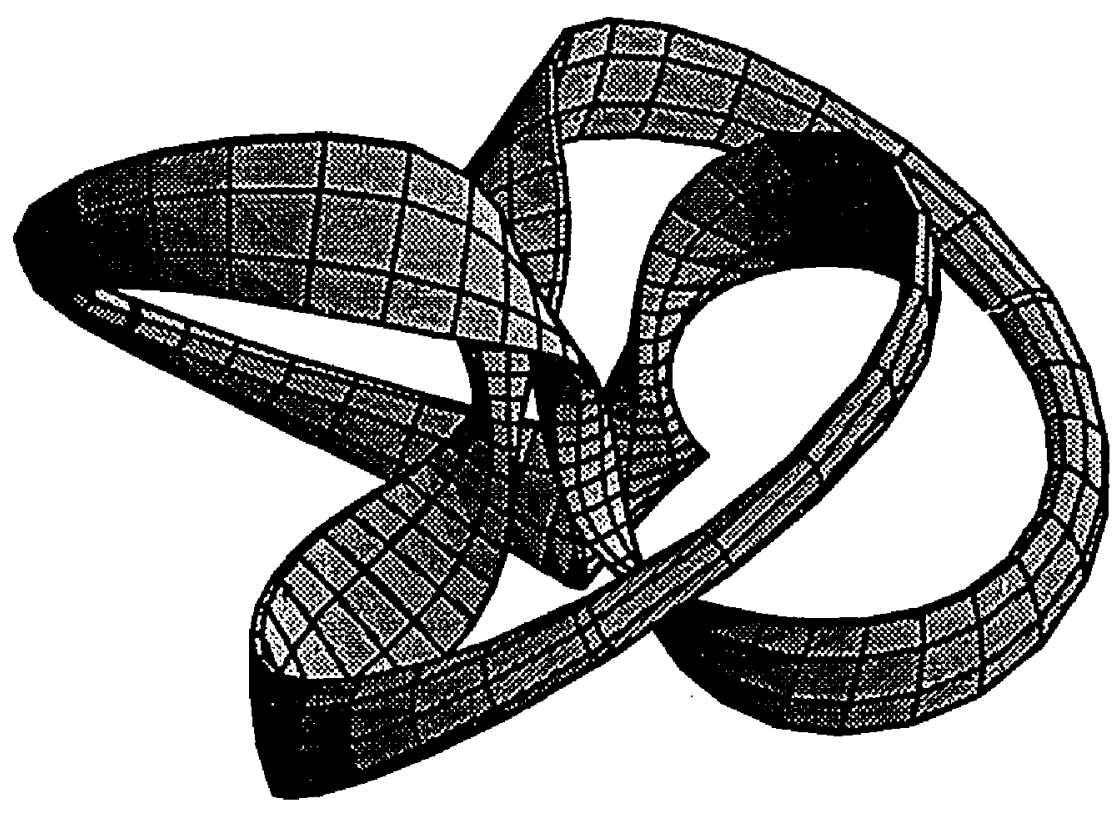

A "tighter" three-period knotted configuration in which the quasihelically symmetric flux surfaces are less flattened than those of Fig. 13. The parameters for this configuration are given by $\{N=3$. $M=2, \zeta=3 / 4, \delta(\varphi)=1.25, l_{0}=0.565$ \} within Eqs. (3.9) and (4.79). 


\section{FIGURE 17}

\section{CURVATURE AND TORSION OF TIGHTER KNOTTED AXIS}

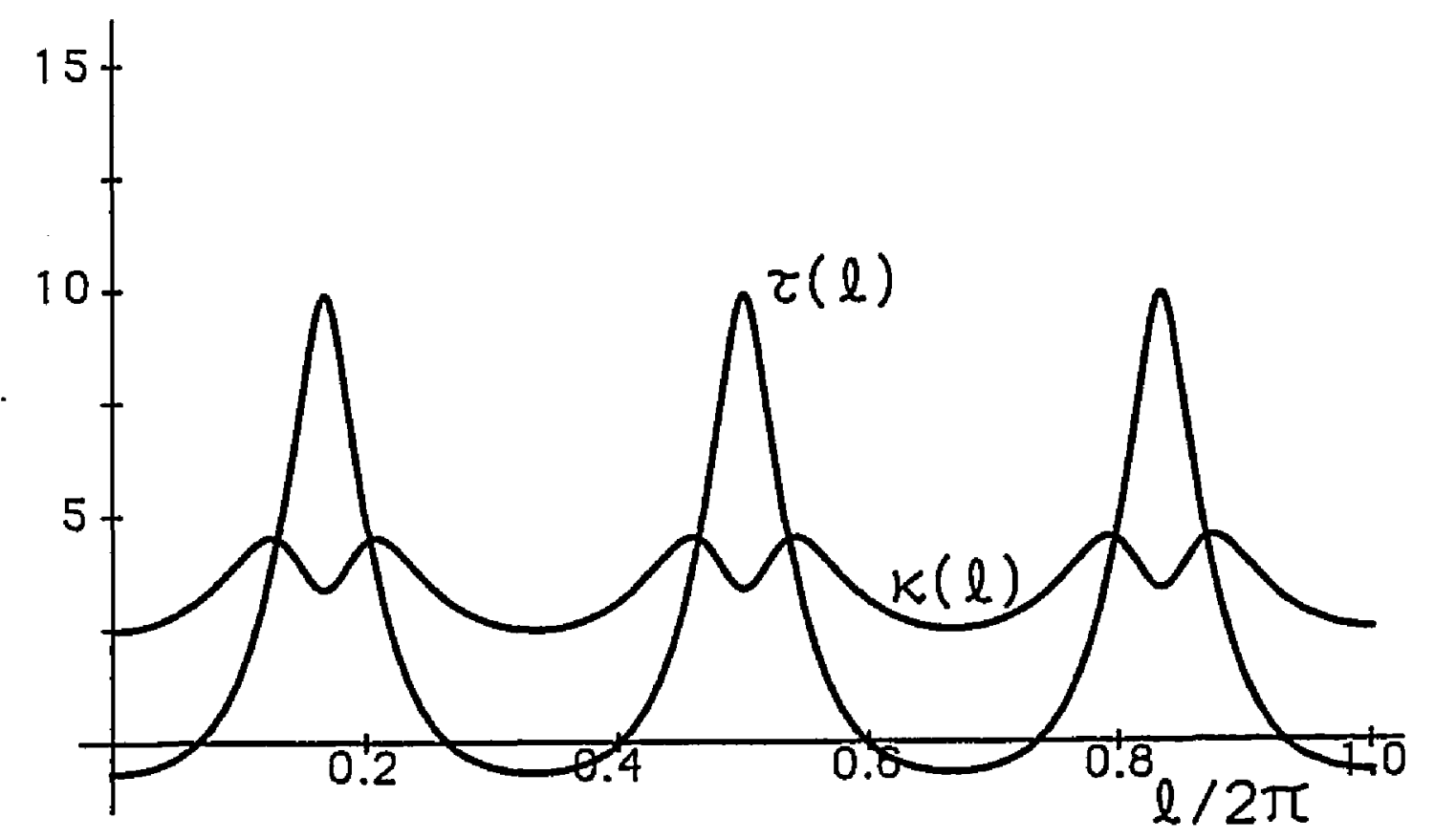

The curvature and torsion corresponding to the "tighter" knot of Fig. 16 , with axis length $L=2 \pi$. 


\section{FIGURE 18}

\section{FIVE PERIOD KNOTTED CONFIGURATION}

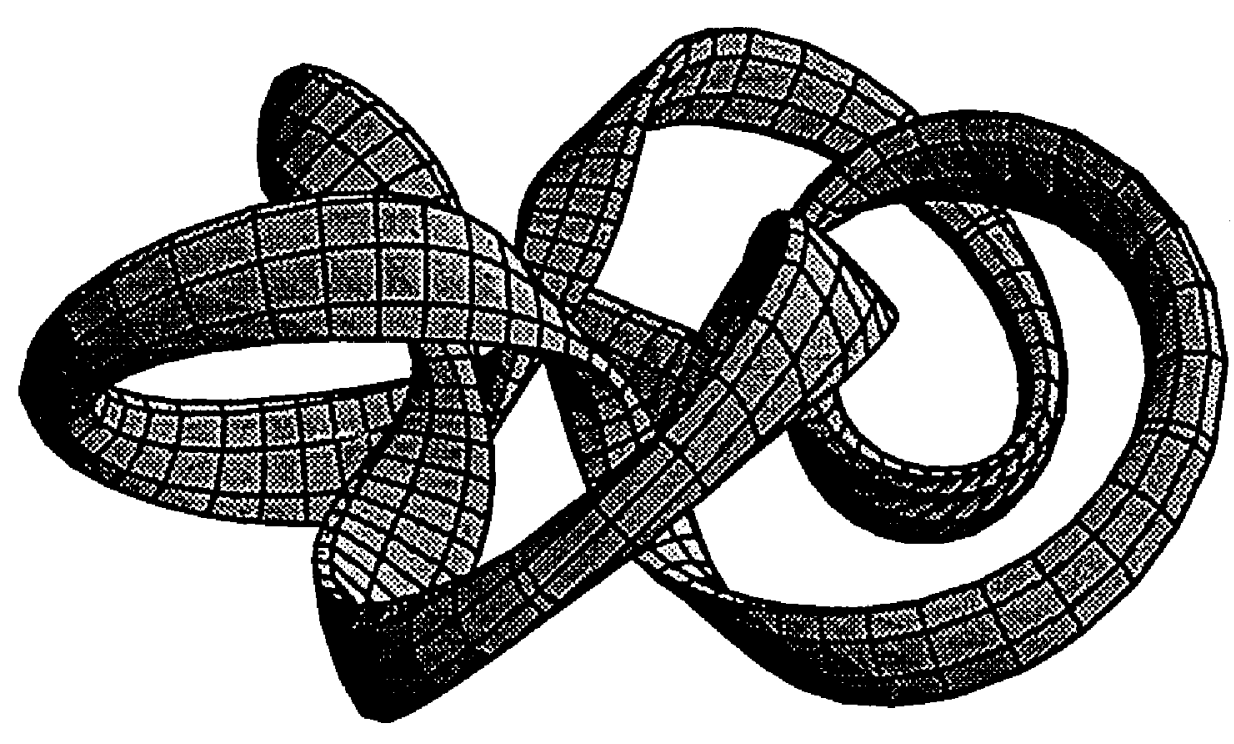

A flux surface corresponding to a five-period knotted configuration which is quasi-helically symmetric through first order. Its parameters are given by $\{N=5, \quad M=2, \quad \zeta=1 / 2, \quad \delta(\varphi)=1.25$, $\left.l_{0}=1.671\right\}$ within Eqs. (3.9) and (4.79). 
FIGURE 19

CURVATURE AND TORSION OF FIVE PERIOD KNOTTED AXIS

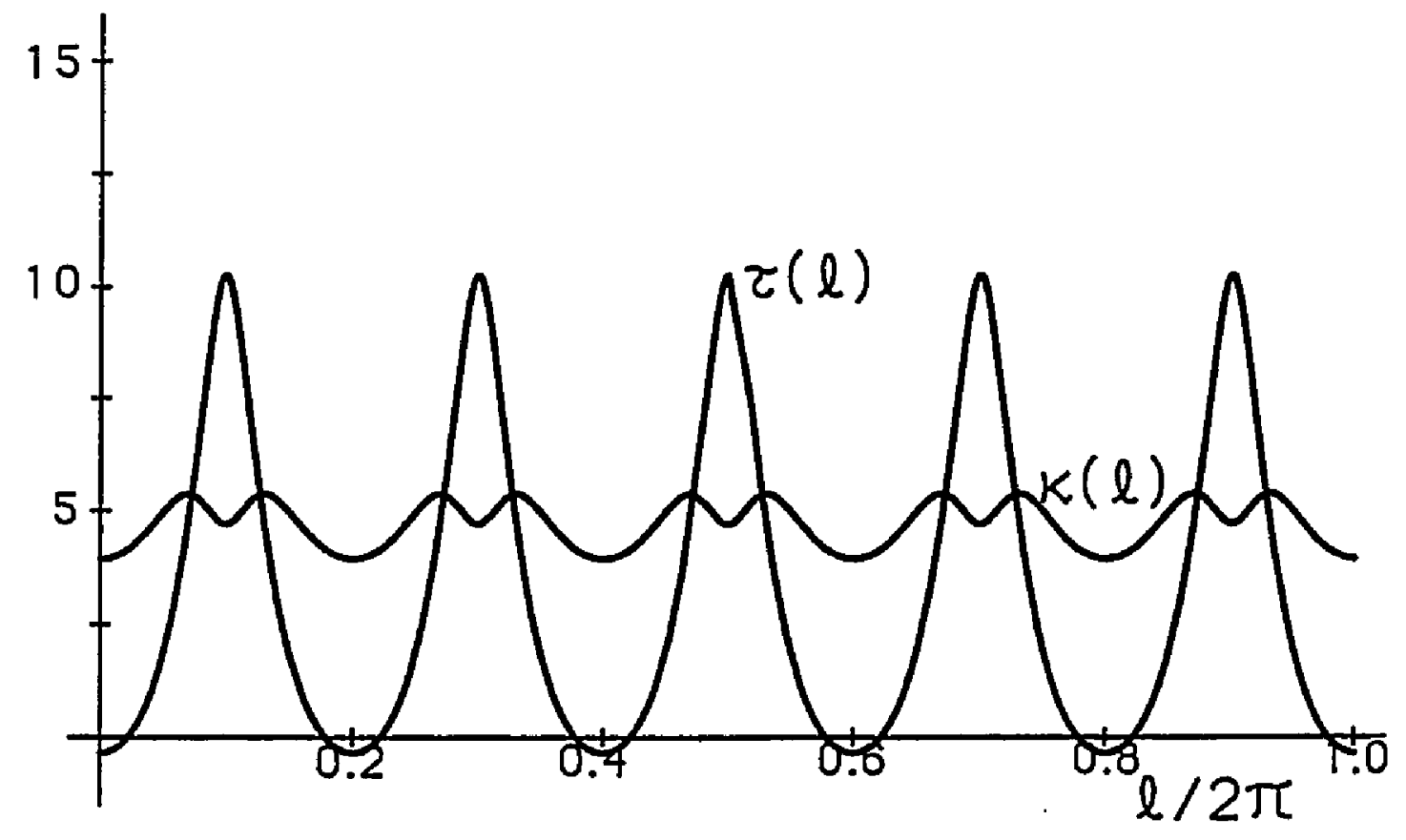

The curvature and torsion corresponding to the five-period knotted configuration of Fig. 18, with axis length $L=2 \pi$. 
symmetry through second order. Hence, we cannot apply the simplistic procedure that was used for first order to obtain quasihelical symmetry through second order. Axis optimizations, which tend to be rather difficult computationally, must be applied.

The equations governing quasi-helical symmetry through second order are presented within the Appendix. Nineteen firstorder, coupled, linear differential equations must be simultaneously integrated 134 , using an optimization of eight independent parameters to insure that the nineteen integrated variables are all periodic in $\varphi$. The task of constructing computational solutions of quasi-helical symmetry through second order is beyond the scope of this dissertation. All of the equations involved are clearly given within the Appendix.

\section{VI-D-3. Quasi-Helically Symmetric Bounding Flux Surface}

Nührenberg and Zille135 optimized the boundary of stellarator configurations to minimize all but one Fourier harmonic within the magnetic field strength. They referred to such stellarators as possessing "quasi-helically symmetry." The bounding magnetic flux surfaces approximate quasi-helical symmetry to an accuracy of better than one percent. For example, one optimization gave a sixperiod configuration with boundary ripple perturbing quasi-helical symmetry on the order of one half of a percent for an aspect ratio of twenty. However, only five poloidal and five toroidal harmonics

${ }^{134}$ Garren, D. A., and Boozer, A. H. (1991). Phys. Fluids B: Plasma Physics 3, 2822. 135Nührenberg, J., and Zille, R. (1988). Phys. Lett. A. 129, 113. 
were used within their optimization. Some of the resulting fifth order Fourier harmonics were non-zero. If Nührenberg and Zille had been able to implement an infinite number of harmonics, then exact quasi-helical symmetry on the bounding surface would have been achieved, but its three dimensional interior would have been only approximately quasi-helically symmetric. In fact, if $\varepsilon_{a}$ corresponds to the flux surface on which quasi-helical symmetry is imposed, then the breaking of quasi-helical symmetry within the interior scales as $\left\{\left(\varepsilon_{a}\right)^{2}-\varepsilon^{2}\right\} \varepsilon$, rather than $\varepsilon^{3}$, as explained within Sec. VI-C. 


\section{CHAPTER VII DISCUSSION}

We investigate toroidal plasma equilibria in which the regions of constant plasma pressure form perfectly nested toroidal surfaces. The magnetic field corresponding to such an equilibrium is forced to have both its contravariant and covariant representations in terms of Boozer coordinates ${ }^{136}$, with the additional requirement that a scalar force balance equation hold ${ }^{137}$. These constraints are equivalent to specifying a toroidal, scalar-pressure, MHD equilibrium in terms of Boozer coordinates. The application of Boozer coordinates offers the advantage of giving guiding-center drift trajectories and associated plasma transport which are determined ${ }^{138}$ by the magnetic field strength as a function of these coordinates. The spatial position is defined in terms of these coordinates, i.e., $x(\psi, \theta, \varphi)$, which permits easy evaluation of the coordinate surfaces and facilitates the expansion about the magnetic axis.

We construct toroidal plasma equilibria by performing a Taylor-Fourier expansion about a general magnetic axis using Boozer coordinates. The magnetic field is assumed to be analytic, so that ${ }^{136}$ Boozer, A. H. (1981). Phys Fluids 24, 1999.

137Garren, D. A., and Boozer, A. H. (1991). Phys. Fluids B: Plasma Physics 3, 2805. ${ }^{138}$ Boozer, A. H. (1984). Phys. Fluids 27, 2441. 
the relevant functions of position are Taylor expanded in terms of the square root of the toroidal flux, $\psi^{1 / 2}$, and Fourier expanded in terms of the poloidal angle, $\theta$, and the toroidal angle, $\varphi$. The Taylor series expansion in $\psi^{1 / 2}$ is equivalent to a power series expansion in terms of a generalized inverse aspect ratio, $\varepsilon$, which is approximately the minor plasma radius, $r$, over the maximum radius of curvature of the axis, $R_{c}$. We find a specific methodology that must be implemented in constructing toroidal, scalar-pressure, MHD equilibria for each order in the expansion.

The radius of convergence for the expansions that we have considered has not been found, although these expansions well represent the known toroidal equilibria. Even if the expansions converge, it is not obvious that they converge to a true equilibrium. For example, if the equilibrium contained terms of the form $f(x)=\exp (-1 / x)$, then the expansions would converge, but not to the equilibrium. [The Taylor series of $\exp (-1 / x)$ about $x=0$ converges to zero, even though this function is not identically zero.\}

Within this analysis, we have also assumed that the plasma pressure forms perfectly nested toroidal surfaces. In practice, small errors in the magnetic field coils can lead to significant stochastic regions within the magnetic field. Except in cases of symmetry, there also remains the difficulty that the plasma pressure gradient must vanish for values of the toroidal flux, $\psi$, that correspond to rational values of the rotational transform, $L(\psi)$. This result is obvious through examination of Eq. (4.7) for $\beta_{*}(\psi, \theta, \varphi)$, 
which becomes singular if $l(\psi)=n / m$ and $d p / d \psi=0$. However, for practical fusion devices, only the low order rational numbers need be avioded.

We show that the constraints of constructing toroidal plasma equilibria with perfect surfaces prevent arbitrary specification of the magnetic field strength ${ }^{139}$ within a three-dimensional volume through third order in the expansion about the axis. In addition, we prove that the first order curvature term in the magnetic field strength cannot be made to completely vanish for all toroidal configurations with non-vanishing axis field strength. However, the magnetic field strength on one particular flux surface is arbitrary, provided that the curvature term does not completely vanish.

The expansion method developed within this dissertation can be used to clarify the types of toroidal plasma equilibria which are most promising for plasma confinement. These techniques can be used to construct the entire spectrum of three-dimensional equilibria in the vicinity of a magnetic axis. The lowest order terms in the expansion largely determine the stability and transport properties for any equilibrium with aspect ratio on the order of $\varepsilon \approx 1 / 10$. This approximation is generally quite valid since the maximum pressure gradient of most existing stellarator devices corresponds to $\varepsilon \leq 1 / 10$. Thus, the first three orders in the expansion about the axis provide a very accurate description of possible toroidal plasma equilibria, since only terms of order $10^{-3}$ ${ }^{139}$ Garren, D. A., and Boozer, A. H. (1991). Phys. Fluids B: Plasma Physics 3, 2805. 
are neglected. In addition, the accuracy of the drift approximation for most fusion plasmas is only on the order $10^{-3}$ or worse.

The most useful product of this dissertation is not the particular expansion method used to construct toroidal plasma equilibria. The most valuable outcome is the analytic results concerning the posssible forms for the magnetic field strength corresponding to toroidal plasma equilibria. Existing computer codes which construct toroidal equilibria can be modified to take advantage of these analytic results.

We apply the techniques of this dissertation to investigate particularly advantageous plasma configurations. The most desirable class of toroidal equilibria would be isodynamic equilibria ${ }^{140}$, which would exhibit a magnetic field strength which is strictly uniform within the magnetic flux surfaces. However, these equilibria can exist ${ }^{141}$ only in limits in which the magnetic field strength on axis vanishes or the magnetic flux surfaces become open. This result implies that the magnetic field strength of nontrivial toroidal equilibria must have some dependence on the poloidal angle, $\theta$. Hence, the guiding-center drifts corresponding to any toroidal plasma equilibrium must always traverse the magnetic flux surfaces.

The next most advantageous class of toridal equilibria are quasi-helically symmetric stellarator equilibria ${ }^{142}$, in which the 140Palumbo, D. (1968). II Nuovo Cimento X53B, 507.

141 Bernardin, M. P., Moses, R. W., and Tetaronis, J. A. (1986). Phys. Fluids 29, 2605.

142Nührenberg, J., and Zille, R. (1988). Phys. Lett. A.129, 113. 
magnetic field strength depends on only one Fourier harmonic within the magnetic flux surfaces. Such toroidal equilibria have strictly confined guiding-center drift trajectories ${ }^{143}$, just as axisymmetric tokamak equilibria. However, diffusive transport on the order of the gyro-radius to the sytem size would remain, leading to a relatively slow loss of the plasma out of the confinement region.

The restrictions in the magnetic field strength show that quasi-helical symmetry is always broken by terms of third order in $\varepsilon$, and thus exact quasi-helically symmetric equilibria do not exist ${ }^{144}$. However, configurations exist with quasi-helical symmetry on one particular magnetic flux surface, but not within its three-dimensional interior. The breaking of quasi-helical symmetry within the interior of such an equilibrium scales as $\left\{\left(\varepsilon_{a}\right)^{2}-\varepsilon^{2}\right\} \varepsilon$, since a term of order $\left(\varepsilon_{a}\right)^{2} \varepsilon$ must be added to the first order field strength in order to offset the $\varepsilon^{3}$ order symmetry breaking term at the flux surface $\varepsilon=\varepsilon_{\mathrm{a}}$. Such quasi-helically bounding equilibria ${ }^{145}$ offer many of the favorable transport properties typical of axisymmetric tokamak equilibria.

The third order term in the magnetic field strength breaking quasi-helical symmetry is either an $M=1$ poloidal harmonic, $\eta_{1}(\varphi) \cos \left(\theta+\zeta_{1}(\varphi)\right)$, or an $M=3$ harmonic, $\eta_{3}(\varphi) \cos \left(3 \theta+\zeta_{3}(\varphi)\right)$, or some linear combination of the two. The actual form of the functions $\eta_{1}(\varphi), \zeta_{1}(\varphi), \eta_{3}(\varphi)$, and $\zeta_{3}(\varphi)$ depends upon the details of ${ }^{143}$ Boozer, A. H. (1983). Phys. Fluids 26, 496. ${ }^{144}$ Garren, D. A., and Boozer, A. H. (1991). Phys. Fluids B: Plasma Physics 3, 2822. ${ }^{145}$ Beidler, C., et. al. (1990). Fusion Technology: A Journal of the American Nuclear Society 17, 148. 
the configuration. However, we can conclude that the magnitudes of the functions $\eta_{1}(\varphi)$ and $\eta_{3}(\varphi)$ is on the order of the fractional toroidal variation in the shape of the magnetic flux surfaces.

Quasi-helical symmetry is most accurately approximated ${ }^{146}$ by a toroidal "helical-like" configuration with a relatively large number of twists and a relatively small plasma radius, as one might naively expect. More exotic "knotted" configurations can also be used to approximate quasi-helical symmetry. The breaking of quasihelical symmetry can made of order $10^{-3}$ for an inverse aspect ratio of order, $\varepsilon \approx 1 / 10$. In comparison, tokamaks with discrete field coils typically exhibit a magnetic field ripple on the order of $2 \times 10^{-3}$.

Analytic ${ }^{147}$ and computational ${ }^{148}$ evidence suggests that quasi-helical stellarator equilibria exist which have symmetry breaking on the order of that corresponding to tokamak equilibria. However, a contiuous toroidal electric current within the plasma is needed to sustain a tokamak equilibrium ${ }^{149}$, whereas, such a toroidal current is not essential for the existence of nonaxisymmetric stellarator equilibria. Therefore, stellarator equilibria which highly approximate quasi-helical symmetry might provide a solution to the dilemma of constructing a fusion device which exhibits good plasma confinement properties and requires no net toroidal plasma current.

${ }^{146}$ Garren, D. A., and Boozer, A. H. (1991). Phys. Fluids B: Plasma Physics 3, 2822. 147 Ibid.

${ }^{148}$ Beidler, C., et. al. (1990). Fusion Technology: A Journal of the American Nuclear Sociaty 17, 148.

${ }^{149}$ Friedberg, J. P. (1987). Ideal Magneto-Hydrodynamics, Plenum Press, New York, p. 107. 


\section{APPENDIX \\ QUASI-HELICAL SYMMETRY THROUGH SECOND ORDER}

We will present the equations that yield quasi-helically symmetric configurations through second order in $\varepsilon$. Quasi-helically symmetry is most relevant to stellarator equilibria, which exhibit vanishing net toroidal electric current near the axis. Hence, we set the constant $I_{2}$, which is a measure of the net toroidal current on axis, to zero in the following analysis.

We will demonstrate that the equations governing quasihelical symmetry through first and second orders must be solved concurrently150. To generate quasi-helical configurations through second order in a convenient manner, we should employ the reciprocal of the square of the magnetic field strength, $1 / B^{2}(\psi, \theta, \varphi)$, in lieu of the field strength itself, $B(\psi, \theta, \varphi)$. Thus, we first reiterate the equations of quasi-helical symmetry through first order using $1 / B^{2}(\psi, \theta, \varphi)$.

The first order set of constraint equations is given by the $\hat{b}_{0}$ constraint at $\varepsilon^{0}$ order and the $\hat{\kappa}_{0}$ and $\hat{z}_{0}$ constraints at $\varepsilon^{1}$ order, as explained within Sec. IV-(E-G). These equations permit the magnetic field strength to be expressed in the quasi-helical form

${ }^{150}$ Garren, D. A., and Boozer, A. H. (1991). Phys. Fluids B: Plasma Physics 3, 2822. 


$$
1 / B^{2}(\psi, \alpha)=\left\{1 / B_{0}\right\}^{2}\left\{1-\sqrt{8}^{\prime} \varepsilon \bar{\eta} \cos (\alpha)\right\}+\cdots,
$$

through first order in $\varepsilon$, with the helical coordinate $\alpha \equiv \theta-N \varphi$, in terms of Boozer coordinates. The configuration through first order is determined by the spatial position of Eq. (4.18). The functions $\left\{X(\psi, \theta, \varphi), Y\left(y^{\prime}, \theta, \varphi\right), Z(\psi, \theta, \varphi)\right\}$ of the spatial position are assumed to have the form given within Eqs. (4.30)-(4.34), with the poloidal angle, $\theta$, replaced by the helical angle, $\alpha$. For a configuration exhibiting a magnetic field strength of the form of Eq. (A.1), the coefficients of Eqs. (4.30)-(4.34) are

$$
\begin{gathered}
X_{1,1 s}(\varphi)=0, \\
X_{1,1 c}(\varphi)=\sqrt{2}^{\prime} \bar{\eta} / k(\varphi), \\
Y_{1,1 s}(\varphi)=\sqrt{2}^{\prime} k(\varphi) / \bar{\pi}, \\
Y_{1,1 c}(\varphi)=\sqrt{2} \sigma(\varphi) k(\varphi) / \bar{\pi} .
\end{gathered}
$$

The function $\sigma(\varphi)$ of Eq. (A.5) is determined by the differential equation

$$
d \sigma / d \varphi=2 G_{0} \tau(\varphi)\{\pi / K(\varphi)\}^{2}-\left\{l_{0}-N\right\}\left\{1+[\pi / K(\varphi)]^{4}+[\sigma(\varphi)]^{2}\right\},
$$

with the periodicity condition $\sigma(\varphi=0)=\sigma(\varphi=2 \pi)$, as in Eq. (4.83).

The first order set of matching constraints also determines the function $Z(\Psi, \theta, \varphi)$ through second order in $\varepsilon$. Recall that its first order term, $Z_{1}(\theta, \varphi)$, vanishes exactly for general toroidal MHD 
equilibria, as derived within Sec. IV-E. The second order term, $Z_{2}(\theta, \varphi)$, is given by

$$
\begin{gathered}
Z_{2,0}(\varphi)=-\left\{1 /\left[8 G_{0}\right]\right\}\left(d V_{1} / d \varphi\right), \\
Z_{2,2 s}(\varphi)=-\left\{1 /\left[8 G_{0}\right]\right\}\left\{\left(d V_{2} / d \varphi\right)-2\left[l_{0}-N\right] V_{3}(\varphi)\right\}, \\
Z_{2,2 c}(\varphi)=-\left\{1 /\left[8 G_{0}\right]\right\}\left\{\left(d V_{3} / d \varphi\right)+2\left[2_{0}-N\right] V_{2}(\varphi)\right\},
\end{gathered}
$$

with

$$
\begin{aligned}
V_{1}(\varphi) \equiv & \left\{X_{1,1 c}(\varphi)\right\}^{2}+\left\{Y_{1,1 s}(\varphi)\right\}^{2}+\left\{Y_{1,1 c}(\varphi)\right\}^{2} \\
& V_{2}(\varphi) \equiv 2 Y_{1,1 s}(\varphi) Y_{1,1 c}(\varphi) \\
V_{3}(\varphi) \equiv & \left\{X_{1,1 c}(\varphi)\right\}^{2}+\left\{Y_{1,1 c}(\varphi)\right\}^{2}-\left\{Y_{1,1 s}(\varphi)\right\}^{2}
\end{aligned}
$$

which are analogous to Eqs. (4.65) -(4.70). Investigation of Eqs. (4.117) and (4.118) reveals that the zeroth order term within the $\beta_{*}(\psi, \theta, \varphi)$ function, i.e., $\beta_{*_{0}}(\varphi)$, vanishes exactly for quasi-helically symmetry through first order, so that it is absent from these first order equations.

The second order set of constraint equations are determined by the $\hat{b}_{0}$ matching constraint at $\varepsilon^{1}$ order and the $\hat{k}_{0}$ and $\hat{\tau}_{0}$ matching constraints at $\varepsilon^{2}$ order. These equations give eight independent constraints on functions of $\varphi$. Sufficient freedom exists within these constraints to yield quasi-helically symmetric equilibria through $\varepsilon^{2}$ order, as explained in Sec. VI-B.

The magnetic field strength of an equilibrium which is quasi- 
helically symmetric through second order has the explicit form ${ }^{151}$

$$
\begin{aligned}
1 / B^{2}(\psi, \alpha)=\left\{1 / B_{0}\right\}^{2}\{1 & -\sqrt{8}^{\prime} \varepsilon \bar{\eta} \cos (\alpha) \\
& \left.+\varepsilon^{2}\{W+C \sin (2 \alpha)+F \cos (2 \alpha)\}+\cdots\right\},
\end{aligned}
$$

with $W, C$, and $F$ constant, in terms of Boozer coordinates. The quasi-helical form of the magnetic field strength in Eq. (A.13) constrains the functions of $\varphi$ within $X_{2}(\theta, \varphi)$ to have the form

$$
\begin{aligned}
& x_{2,0}(\varphi)=\left\{h_{0}(\varphi)-W\right\} /\{2 \kappa(\varphi)\}, \\
& x_{2,2 s}(\varphi)=\left\{h_{2 s}(\varphi)-c\right\} /\{2 \kappa(\varphi)\}, \\
& x_{2,2 c}(\varphi)=\left\{h_{2 c}(\varphi)-F\right\} /\{2 k(\varphi)\},
\end{aligned}
$$

with

$$
\begin{aligned}
h_{0}(\varphi) \equiv & 2 p_{2}+\left\{2 / G_{0}\right\}\left(d z_{2,0} / d \varphi\right) \\
& +\left\{\left\{q_{c}(\varphi)\right\}^{2}+\left\{r_{c}(\varphi)\right\}^{2}+\left\{s_{c}(\varphi)\right\}^{2}+\left\{q_{s}(\varphi)\right\}^{2}+\left\{r_{s}(\varphi)\right\}^{2}\right\} /\left\{2\left\{G_{0}\right\}^{2}\right\} \\
h_{2 s}(\varphi) \equiv & \left\{2 / G_{0}\right\}\left\{\left(d Z_{2,2 s} / d \varphi\right)-2\left\{l_{0}-N\right\} Z_{2,2 c}(\varphi)\right\} \\
& +\left\{q_{s}(\varphi) q_{c}(\varphi)+r_{s}(\varphi) r_{c}(\varphi)\right\} /\left\{G_{0}\right\}^{2} \\
h_{2 c}(\varphi) \equiv & \left\{2 / G_{0}\right\}\left\{\left(d Z_{2,2 c} / d \varphi\right)+2\left\{L_{0}-N\right\} Z_{2,2 s}(\varphi)\right\} \\
& +\left\{\left\{q_{c}(\varphi)\right\}^{2}+\left\{r_{c}(\varphi)\right\}^{2}+\left\{s_{c}(\varphi)\right\}^{2}-\left\{q_{s}(\varphi)\right\}^{2}-\left\{r_{s}(\varphi)\right\}^{2}\right\} /\left\{2\left\{G_{0}\right\}^{2}\right\}
\end{aligned}
$$

and

${ }^{151}$ Garren, D. A., and Boozer, A. H. (1991). Phys. Fluids B: Plasma Physics 3, 2822. 


$$
\begin{gathered}
q_{s}(\varphi) \equiv-\left\{l_{0}-N\right\} X_{1,1 c}(\varphi)+G_{0} \tau(\varphi) Y_{1,1 s}(\varphi), \\
q_{c}(\varphi) \equiv\left(d X_{1,1 c} / d \varphi\right)+G_{0} r(\varphi) Y_{1,1 c}(\varphi), \\
r_{s}(\varphi) \equiv\left(d Y_{1,1 s} / d \varphi\right)-\left\{l_{0}-N\right\} Y_{1,1 c}(\varphi), \\
r_{c}(\varphi) \equiv\left(d Y_{1,1 c} / d \varphi\right)+\left\{l_{0}-N\right\} Y_{1,1 s}(\varphi)-G_{0} \tau(\varphi) X_{1,1 c}(\varphi), \\
s_{c}(\varphi) \equiv \sqrt{2} G_{0} \bar{\eta} .
\end{gathered}
$$

Four of the eight equations of the second order set of matching constraints determine the form of the function $Z_{3}(\theta, \varphi)$ via the functions $Z_{3.1 \mathrm{~s}}(\varphi), Z_{3.1 \mathrm{c}}(\varphi), Z_{3,3 \mathrm{~s}}(\varphi)$, and $Z_{3,3_{c}}(\varphi)$. These functions of $\varphi$ partially determine the configuration through third order. We are only concerned with the form of the configuration through second order, so that these functions of $\varphi$ within $Z_{3}(\theta, \varphi)$ will not be explicitly given.

Two of the four remaining second order $\varphi$-constraints can be expressed as relations for $Y_{2,2 s}(\varphi)$ and $Y_{2,2 c}(\varphi)$ via

$$
\begin{aligned}
& Y_{2,2 s}(\varphi)=-k(\varphi)-\{k(\varphi) / \bar{\eta}\}^{2}\left\{X_{2,0}(\varphi)+X_{2,2 c}(\varphi)-\sigma(\varphi) X_{2,2 s}(\varphi)\right\}, \\
& Y_{2,2 c}(\varphi)=Y_{2,0}(\varphi)+\{k(\varphi) / \bar{\eta}\}^{2}\left\{X_{2,2 s}(\varphi)-\sigma(\varphi)\left\{X_{2,0}(\varphi)-X_{2,2 c}(\varphi)\right\}\right\} .
\end{aligned}
$$

The two remaining equations of the second order set of matching constraints are given by

$$
x_{1,1 c}(\varphi) f_{x, 2 s}(\varphi)+Y_{1,1 c}(\varphi) f_{y, 2 s}(\varphi)-Y_{1,1 s}(\varphi)\left\{f_{y, 2 c}(\varphi)+f_{y, 0}(\varphi)\right\}=0,
$$




$$
\begin{aligned}
X_{1,1 c}(\varphi)\left\{f_{x, 2 c}(\varphi)-f_{x, 0}(\varphi)\right\} & +Y_{1,1 s}(\varphi) f_{y, 2 s}(\varphi) \\
& +Y_{1,1 c}(\varphi)\left\{f_{y, 2 c}(\varphi)-f_{y, 0}(\varphi)\right\}=0,
\end{aligned}
$$

with

$$
\begin{aligned}
& f_{x, 0}(\varphi) \equiv\left(d x_{2,0} / d \varphi\right) \\
& +G_{0}\left(\tau(\varphi) Y_{2,0}(\varphi)+k(\varphi) Z_{2,0}(\varphi)\right. \\
& -2 Z_{2,2 s}(\varphi) Y_{2,2 c}(\varphi)+2 Z_{2,2 c}(\varphi) Y_{2,2 s}(\varphi) \\
& \left.-\left\{\sqrt{2} p_{2} G_{0} \bar{\eta} /\left\{L_{0}-N\right\}\right\} Y_{1,1 c}(\varphi)\right\} \text {, } \\
& f_{X, 2 s}(\varphi) \equiv\left(d X_{2,2 s} / d \varphi\right)-\left\{l_{0}-N\right\} X_{2,2 c}(\varphi) \\
& +G_{0}\left\{\tau(\varphi) Y_{2,2 s}(\varphi)+k(\varphi) Z_{2,2 s}(\varphi)\right. \\
& -2 Z_{2,0}(\varphi) Y_{2,2 c}(\varphi)+2 Z_{2,2 c}(\varphi) Y_{2,0}(\varphi) \\
& \left.+\left\{\sqrt{2}{ }^{\prime} P_{2} G_{0} \bar{\eta} /\left\{L_{0}-N\right\}\right\} Y_{1,1 s}(\varphi)\right\} \\
& f_{x, 2 c}(\varphi) \equiv\left(d X_{2,2 c} / d \varphi\right)+\left\{2_{0}-N\right\} X_{2,2 s}(\varphi) \\
& +G_{0}\left\{\tau(\varphi) Y_{2,2 c}(\varphi)+k(\varphi) Z_{2,2 c}(\varphi)\right. \\
& -2 Z_{2,2 s}(\varphi) Y_{2,0}(\varphi)+2 Z_{2,0}(\varphi) Y_{2,2 s}(\varphi) \\
& \left.+\left\{\sqrt{2} P_{2} G_{0} \bar{\eta} /\left\{L_{0}-N\right\}\right\} Y_{1,1 c}(\varphi)\right\} \text {, } \\
& -G_{0}\left\{\tau(\varphi) X_{2,0}(\varphi)-2 Z_{2,2 s}(\varphi) X_{2,2 c}(\varphi)+2 Z_{2,2 c}(\varphi) X_{2,2 s}(\varphi)\right. \\
& \left.-\left\{\sqrt{2} p_{2} G_{0} \bar{\eta} /\left\{l_{0}-N\right\}\right\} X_{1,1 c}(\varphi)\right\} \text {, }
\end{aligned}
$$




$$
\begin{aligned}
& f_{y, 2 s}(\varphi) \equiv\left(d Y_{2,2 s} / d \varphi\right)-\left\{l_{0}-N\right\} Y_{2,2 c}(\varphi) \\
& -G_{0}\left\{\tau(\varphi) X_{2,2 s}(\varphi)-2 Z_{2,0}(\varphi) X_{2,2 c}(\varphi)+2 Z_{2,2 c}(\varphi) X_{2,0}(\varphi)\right. \\
& \left.+\left\{\sqrt{2} p_{2} G_{0} \bar{\eta} /\left\{2_{0}-N\right\}\right\} X_{1,1 s}(\varphi)\right\} \text {, } \\
& f_{X, 2 c}(\varphi) \equiv\left(d Y_{2,2 c} / d \varphi\right)+\left\{l_{0}-N\right\} Y_{2,2 s}(\varphi) \\
& -G_{0}\left(\tau(\varphi) X_{2,2 c}(\varphi)\right)-2 Z_{2,2 s}(\varphi) X_{2,0}(\varphi)+2 Z_{2,0}(\varphi) X_{2,2 s}(\varphi) \\
& \left.+\left\{\sqrt{2} P_{2} G_{0} \bar{\eta} /\left\{\imath_{0}-N\right\}\right\} X_{1.1 c}(\varphi)\right\} \text {, }
\end{aligned}
$$

In summary, toroidal plasma equilibria which are quasihelically symmetric through second order in $\varepsilon$ must satisfy 152 the three non-linear differential equations of Eqs. (A.6), (A.27), and (A.28). All $\varphi$-dependence within these three equations can be expressed in terms of the four $\varphi$-functions $\tau(\varphi), k(\varphi), \sigma(\varphi)$, and $Y_{2,0}(\varphi)$, and their derivatives of various orders. Herein lies the difficulty in constructing quasi-helically symmetric equilibria through second order.

Finding general toroidal equilibria through second order is quite manageable. First, one must choose a magnetic axis about which to perform the expansion. First-order flux surfaces are obtained by integrating one non-linear, first-order differetial equation similar to that of Eq. (A.6). A computational optimization in one dimension must be performed to insure that the integrated function of $\varphi$ is periodic. Second order corrections to these first order flux surfaces are then obtained by integrating two non-linear, first-order differential equations similar to those of Eqs. (A.27) and ${ }^{152}$ Garren, D. A., and Boozer, A. H. (1991). Phys. Fluids B: Plasma Physics 3, 2822. 
(A.28). An optimization in two dimensions is required to insure that both integrated functions of $\varphi$ are also periodic.

Obtaịning quasi-helically symmetric equilibria through second order is considerably more difficult. First, three of the four functions, $K(\varphi), \tau(\varphi), \sigma(\varphi)$, and $Y_{2,0}(\varphi)$ must be used to solve the set of differential equations given by Eqs. (A.6), (A.27), and (A.28). Thus, at least one of the functions $k(\varphi)$ or $\tau(\varphi)$, which determine the shape of magnetic axis, must be optimized in order to satisfy this set of differential equations. If one of these two functions, $k(\varphi)$ or $\tau(\varphi)$, is given, then the other can obviously be varied to satisfy the three differential equations of Eqs. (A.6), (A.27), and (A.28). However, the probablity that the resulting set of $k(\varphi)$ and $\tau(\varphi)$ is consistent with a closed curve occupies a set of measure zero. Hence, a simultaneous optimization 153 of the curve to "bite its own tail," in addition to an optimization of Eqs. (A.6), (A.27), and (A.28), is required in order to find quasi-helical symmetric configurations through second order in the expansion.

Optimizing an arbitrary curve to close on itself requires integration of the Frenet equations of Eqs. (3.1)-(3.4), with the periodicity condition that all components of the vectors $r_{0}(\varphi)$, $\hat{\boldsymbol{K}}_{0}(\varphi), \hat{\tau}_{0}(\varphi)$, and $\hat{b}_{0}(\varphi)$ be periodic in $\varphi$. We can write these vectors in terms of the toroidal angle, $\varphi$, instead of the axis length, $\ell$, since these two variables are directly proportional for uniform magnetic field strength on axis. Axis optimizations yielding a closed curve can be obtained by varying only two-Fourier harmonics within a set ${ }^{153}$ Garren, D. A., and Boozer, A. H. (1991). Phys. Fluids B: Plasma Physics 3, 2822. 
of $k(\varphi)$ and $\tau(\varphi)$, as developed within Sec. II-C.

A second complication 154 of computationally constructing quasi-helically symmetric equilibria through second order is that Eqs. (A.27) and (A.28) involve third derivatives of the functions $k(\varphi)$ and $\sigma(\varphi)$. Implementing standard techniques for integrating differential equations, the three differential equations of Eqs. (A.6), (A.27), and (A.28) are equivalent to seven independent, non-linear, first-order differential equations. An optimization in six dimensions is required in order to find initial conditions which solve these seven first-order equations, assuming one parameter can be eliminated as an arbitrary phase of integration. This optimization of six independent parameters to solve seven first-order differential equations must be coupled with the optimization of two independent parameters used to satisfy the twelve first-order differential equations of the Frenet equations. Thus, an integration of nineteen first order differential equations using an optimization of eight independent parameters is required in order to construct quasi-helically symmetric equilibria through second order in the expansion parameter, $\varepsilon$.

We do not intend to present the methods developed within this dissertation as a practical means for constructing toroidal equilibria through second and higher orders. Many computational codes which find equilibria with desirable stability and transport properties already exist. For example, Nührenberg and Zille ${ }^{155}$

${ }^{154}$ Garren, D. A., and Boozer, A. H. (1991). Phys. Fluids B: Plasma Physics 3, 2822. ${ }^{155}$ Nührenberg, J., and Zille, R. (1988). Phys. Lett. A. 129, 113. 
construct equilibria which highly approximate quasi-helical symmetry using a minimization of all but one Fourier harmonic of the magnetic field strength on a particular magnetic surface. The primary application of this dissertation is the insight received from such an analytic investigation. Computaional codes developed in the future can exploit the analytic results that we have obtained. 


\section{BIBLIOGRAPHY}

Bateman, G. (1978). MHD Instabilities, MIT Press, Cambridge, Massachusetts, and London, England.

Beidler, C., et. al. (1990). Fusion Technology: A Journal of the American Nuclear Society 17, 148.

Bernardin, M. P., Moses, R. W., and Tetaronis, J. A. (1986) Phys. Fluids 29, 2605.

Boozer, A. H. (1981). Phys Fluids 24, 1999.

Boozer, A. H. (1983). Phys. Fluids 26, 496.

Boozer, A. H. (1984). Phys. Fluids 27, 2441.

Boozer, A. H. (1990). submitted to Phys. Fluids.

Boozer, A. H., and Gardner, H. (1991). Phys Fluids B: Plasma Physics 2, 2408.

Friedberg, J. P. (1987). Ideal Magneto-Hydrodynamics, Plenum Press, New York.

Garren, D. A., and Boozer, A. H. (1991). Phys. Fluids B: Plasma Physics 3, 2805.

Garren, D. A., and Boozer, A. H. (1991). Phys. Fluids B: Plasma Physics 3, 2822.

Goldstein, H. (1980). Classical Mechanics, Addison-Wesley Publishing Company, Inc., Philippines, 2nd ed. 


\section{Bibliograpiny Continued:}

Kruskal, M. (1957). Rendiconti del Terzo Congresso Internazionale sui Fenomeni D'Ionizzazione nei Gas tenuto a Venazia (Societa Italiana di Fisica, Milan, p. 56.

Kruskal, M. D., and Kulsrud, R. M. (1958). Phys. Fluids 1, 265.

Kuo-Petravic, G., and Boozer, A. H. (1987). Comp. Physics 73, 107.

Lawson, J. D. (1957). Proc. Phys. Soc. London, Sec. B70, 6.

Lortz, D., and Nührenberg, J. (1976). Z. Naturforsch. 31a, 1277.

Lortz, D., and Nührenberg, J. (1977). Nucl. Fusion 17, 125.

Lortz, D., and Nührenberg, J. (1979). Z. Naturforsch. 34a, 167.

Mathews, J., and Walker, R. L., (1970). Mathematical Methods of Physics, W. A. Benjamin, Inc., New York, 2nd ed., p. 408.

Mercier, C. (1964). Nucl. Fusion 4, 213.

Nishkawa, K., and Wakatani, M. (1990). Plasma Physics: Basic Theory with Fusion Applications, Springer-Verlag, Berlin, Heidelberg, Germany.

Nührenberg, J., and Zille, R. (1987). Theory of Fusion Plasmas, Proceedings of the workshop held at Villa Cipressi-Varenna, Italy, Aug. 24-28, Societa Italiana di Fisica, Bologna, Italy, p. 3.

Nührenberg, J., and Zille, R. (1988). Phys. Lett. A. 129, 113.

Palumbo, D. (1968). II Nuovo Cimento X53B, 507.

Shafranov, V. D. (1966). Reviews of Plasma Physics, edited by M. A. Leontovich, Consultants Bureau, New York, Vol. 2.

Solov'ev, L. S., and Shafranov, V. D. (1970). Reviews of Plasma Physics, edited by M. A. Leontovich, Consultants Bureau, New York, Vol. 5, p. 1. 
Bibliography Continued:

Spitzer Jr., L. (1958). Phys. Fluids 1, 253.

Taylor, J. B. (1964). Phys. Fluids 7, 767.

White, R. B. (1989). Theory of Tokamak Plasmas, North Holland Physics, Amsterdam, p. 6. 


\section{VITA}

\section{David Alan Garren}

Born in Newport News, Virginia, June 9, 1965. Graduated Validictorian at Salem High School in Salem, Virginia, June, 1983. Graduated Salutatorian at Roanoke College in Salem, Virginia, May, 1986, with B.S. majors in mathematics and physics with a concentration in computer science. Obtained M.S. from the College of William and Mary in Virginia, May, 1988. All requirements except the dissertation have been completed for the Ph.D. degree at the College of William and Mary. 